\title{
CURVES IN GRASSMANNIANS
}

\author{
DAVID PERKINSON
}

\begin{abstract}
Curves in Grassmannians are analyzed using the special structure of the tangent bundle of a Grassmannian, resulting in a theory of inflections or Weierstrass behavior. A duality theorem is established, generalizing the classical duality theorem for projective plane curves. The appendices summarize basic information about principal parts bundles and their application to studying the inflections of curves in projective space.
\end{abstract}

\section{INTRODUCTION}

This paper develops Joe Harris's idea for classifying curves in Grassmannians based on the special structure of the tangent bundle of a Grassmannian. A map of a curve, $X$, into a Grassmannian is given by a vector space, $V$, of globally spanning sections of some vector bundle, $E$, on $X$. To this, we associated a sequence of vector bundle quotients

$$
V_{X}=V \otimes \mathscr{O}_{X} \rightarrow E \rightarrow E_{1} \rightarrow E_{2} \rightarrow \ldots
$$

called derived bundles and define higher differential ranks and torsion sheaves. Our goal is then to explain the geometry behind these constructions. The main tools we use are principal parts bundles and the closely related osculating bundles, in the spirit of Piene's work [Pi1]. Main facts about these bundles are relegated to the appendices and are assumed throughout the main body of the paper.

Derived bundles are defined in $\S 1$ and their connection with principal parts bundles is presented in $\S 2$. Piene's osculating bundles appear in $\S 4$. They are used to formulate a key result, Theorem 4.2 , which states that the surjections between derived bundles, $(*)$, lift to give surjections between osculating bundles

$$
V_{X} \rightarrow E \rightarrow \mathrm{G}^{1}\left(E_{1}\right) \rightarrow \mathrm{G}^{2}\left(E_{2}\right) \rightarrow \ldots
$$

This property is used to characterize derived bundles in Theorem 4.6. It is also the key idea behind Theorem 5.1, which is a refinement of the normal form for a curve in a Grassmannian due to Griffiths and Harris, [GH2].

The normal form for curves in Grassmannians can be interpreted to explain the geometric meaning of derived bundles and differential ranks. Locally, a curve in a Grassmannian is given by the span of vectors parametrized by the curve. It might happen that some of these vectors are derivatives of others.

Received by the editors August 15, 1994.

1991 Mathematics Subject Classification. Primary 14H60, 14H55, 14 M15. 
Roughly, the differential rank is the minimum number of vectors needed such that they, along with their derivatives up to various orders, determine the map to the Grassmannian. The higher differential ranks express the orders of the derivatives. This is made precise by Theorem 5.1.

In certain situations, a calculation of ranks will show that the surjections to the osculating bundles in $(* *)$ are isomorphisms. This is the idea behind Theorem 6.2.1, which is used to recover a result of Griffiths and Harris, [GH2, p. 386] characterizing curves with differential rank one: each comes from a curve in projective space by taking a cone over an associated map of some order. An associated map of order $t$ for a curve in projective space sends a point on the curve to its $t$-th osculating space (the space spanned by the derivatives of order $\leq t$ of a local parametrization of the curve).

The formalism of our vector bundle constructions suggested one of the main results of the paper, a duality theorem for curves in Grassmannians, Theorem 7.1. In the case where $E$ is an osculating bundle for a curve in projective space, this result specializes to give Piene's duality theorem, [Pil], which is the modern expression of the classical duality theorem for curves in projective space. (The most special case is the fact that the double dual of a projective plane curve is the curve, itself.) As an application, we discuss the birationality of the associated maps, (Proposition 3.3.1).

The torsion sheaves measure the inflectionary behavior of a curve in a Grassmannian. In the special case of a curve in projective space, their lengths are known as stationary indices: these are the numbers appearing in the generalized Plücker formulas describing the way a curve flexes.

The paper ends with several examples: curves in Grassmannians coming from taking "joins of lines"; curves of degree three; a relation between the degree of a bundle generated by global sections and its possible differential ranks; and the sequence of differential ranks and torsion numbers (lengths of torsion sheaves) possible on the projective line.

E. Ballico has continued the study initiated in this paper, [Ba1], and has generalized some of the results to higher dimensional varieties, [Ba2].

\section{ACKNOWLEDGMENTS}

I would like to thank my graduate school advisor, William Fulton; this paper is based on my Ph.D. thesis. Thanks also to the Matematisk Institutt of the University of Oslo, Norway-especially Ragni Piene-for providing a place to work and inspiration. Finally, thanks to Reed College for time and support through a Vollum research grant.

\section{Derived bundles}

We study a map of a smooth curve over an algebraically closed field, $k$, into the Grassmannian of $r$-dimensional quotients of an $n$-dimensional vector space $V$ over $k$ :

$$
f: X \rightarrow G(V, r)=G_{r-1} \mathbb{P}(V)=G
$$

or equivalently, a surjection

$$
\phi: V_{X}=V \otimes_{k} \mathscr{O}_{X} \rightarrow E
$$


where $E$ is a vector bundle of rank $r$ on $X$. The universal exact sequence on $G$

$$
0 \rightarrow S \rightarrow V_{G} \rightarrow Q \rightarrow 0
$$

where $Q$ is the universal $r$-quotient, pulls back to

$$
0 \rightarrow S_{E} \rightarrow V_{X} \stackrel{\phi}{\longrightarrow} E \longrightarrow 0
$$

where $S_{E}$ is the kernel of $\phi$. The tangent map

$$
T_{X} \rightarrow f^{*} T_{G} \cong f^{*} \mathscr{H} \operatorname{om}(S, Q) \cong \mathscr{H} o m\left(S_{E}, E\right)
$$

is the same as a map

$$
\partial=\partial_{\phi}: S_{E} \rightarrow \Omega_{X / k} \otimes E
$$

where $\Omega_{X / k}=T_{X / k}^{-1}$ is the cotangent bundle.

Definition 1.6. The differential rank of $\phi($ or $f)$ is the rank of the image of $\partial$. The torsion sheaf for $\phi$ (or $f$ ) is the torsion subsheaf of the cokernel of $\partial$.

The differential rank of $\phi$ is the rank of $\partial$ restricted to a generic fiber. At special fibers, the rank of $\partial$ may drop, this being measured by the torsion sheaf.

The map $\partial$ is now used to construct a sequence of related maps to Grassmannians.

Definition 1.7. The first derived bundle of $\phi$ is the vector bundle

$$
E_{1}=\left(\operatorname{cok}(\partial) \otimes \Omega_{X / k}^{-1}\right) / \text { torsion. }
$$

Tensoring the natural map $\Omega_{X / k} \otimes E \rightarrow \operatorname{cok}(\partial)$ by $\Omega_{X / k}^{-1}$ and composing with $\phi$ induces a surjection

$$
\phi_{1}: V_{X} \rightarrow E_{1}
$$

which factors through $\phi$. There is a corresponding map

$$
f_{(1)}: X \rightarrow G\left(V, \operatorname{rank}\left(E_{1}\right)\right) \text {. }
$$

One may now repeat the process with $E_{1}$ in place of $E$. Inductively, define the $i$-th derived bundle of $\phi, E_{i}$, to be the first derived bundle of $\phi_{i-1}$. The bundle $E_{i}$ comes with a surjection

$$
\phi_{i}: V_{X} \rightarrow E_{i}
$$

and a corresponding map

$$
f_{(i)}: X \rightarrow G\left(V, \operatorname{rank}\left(E_{i}\right)\right) .
$$

Define the $i$-th differential rank of $\phi, \operatorname{drk}_{i}(\phi)$, to be the differential rank of $\phi_{i-1}$ and the $i$-th torsion sheaf, tor $_{i}(\phi)$, to be the torsion sheaf of $\phi_{i-1}$. The $i$-th torsion divisor is defined to be $\sum_{x \in X}$ length $\left(\right.$ tor $\left._{i} \phi_{x}\right) \cdot x$ and the $i$-th torsion number is the degree of this divisor.

Thus, letting $\phi_{0}=\phi$, the previously defined differential rank and torsion sheaf of $\phi$ may be called the first differential rank and first torsion sheaf, respectively. In sum, we have associated with each map of $X$ into a Grassmannian, 
a sequence of maps of $X$ into other Grassmannians, a corresponding sequence of surjections

$$
V_{X} \rightarrow E \rightarrow E_{1} \rightarrow E_{2} \rightarrow \cdots
$$

and a sequence of torsion sheaves on $X$. The $i$-th differential rank is

$$
\mathrm{drk}_{i} \phi=\mathrm{rk} E_{i-1}-\mathrm{rk} E_{i} .
$$

Remark 1.9. Tensoring (1.5) by $\Omega_{X / k}^{-1}$ defines a map

$$
T_{X / k} \otimes S_{E} \rightarrow E
$$

whose cokernel modulo torsion is $E_{1}$. We used $\partial$ to define the derived bundles instead of this map because it arises more naturally when using principal parts bundles to study differential ranks, $(\S 2)$.

\section{PRINCIPAL PARTS BUNDLES AND A DESCRIPTION OF $\partial$ IN LOCAL COORDINATES}

Although $\partial: S_{E} \rightarrow \Omega_{X / k} \otimes E$, used to define the derived bundles, was defined by identifying the tangent bundle of the Grassmannian with a space of maps, we will mostly use an alternate description using $\mathrm{P}^{1}(E)$, the first order principal parts of $E$. This is given in Proposition 2.1. The proof of Proposition 2.1 shows that the map $\partial$ is closely related to the second fundamental form homomorphism on the Grassmannian.

The section ends with a description of $\partial$ in local coordinates. It will be used later to state Theorem 5.1 giving the normal form for a curve in a Grassmannian and leads to a geometric explanation of the differential rank.

Proposition 2.1. There is a commutative diagram with exact rows

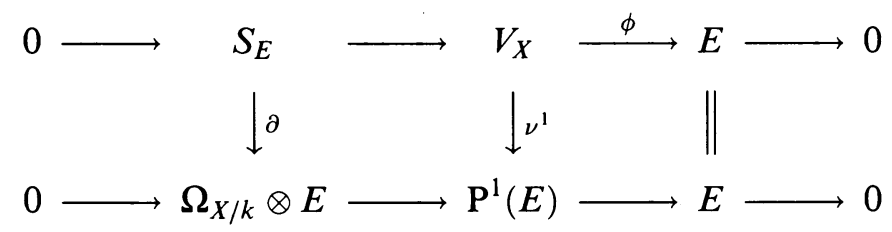

where the bottom row is the fundamental exact sequence and $\nu^{1}$ is the Taylor series map (A.6). Thus, $\partial$ is the Taylor series map $\nu^{1}$ restricted to $S_{E}$.

Proof. The Taylor series map lifts $\phi$, giving a map of exact sequences as shown except we must verify that the induced map $S_{E} \rightarrow \Omega_{X / k} \otimes E$ is $\partial$. There is a diagram similar to (2.2) on the Grassmannian. The map $V_{G} \rightarrow Q$ to the universal $r$-quotient factors through the first order principal parts of $Q$ to give

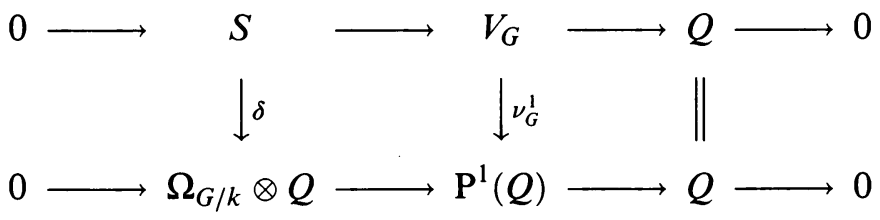

Let $d f: f^{*} \Omega_{G / k} \rightarrow \Omega_{X / k}$ be the cotangent map. Pull back (*) to the curve and use the natural map $f^{*} \mathrm{P}^{1}(Q) \rightarrow \mathrm{P}^{1}(E)$, (A.2.4), to get the commutative 
diagram

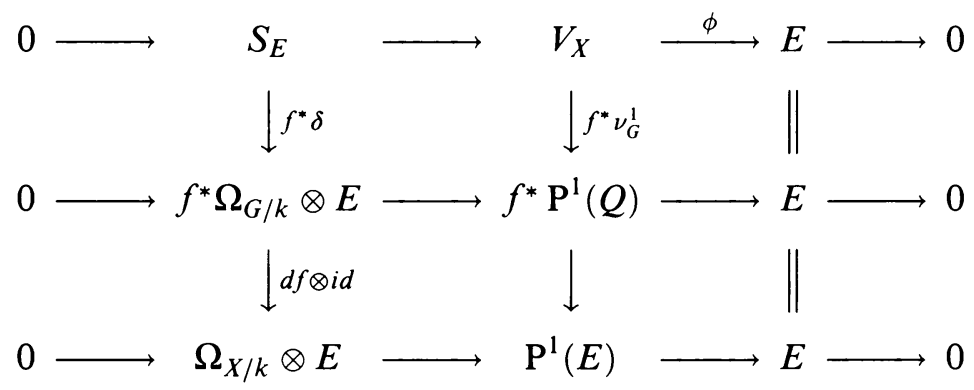

The composite of the middle vertical maps is the Taylor series map $\nu^{1}$; so it suffices to show that the composite of the vertical maps on the left is $\partial$. We see this by noting the connection between $\delta$ and the standard identification: $T_{G} \cong \mathscr{H} \operatorname{om}(S, Q)$. Tensoring the map $\delta$ of $(*)$ by $Q^{*}$ induces a map $\alpha: S \otimes$ $Q^{*} \rightarrow \Omega_{G / k}$ which one may check, using local coordinates, is an isomorphism. The dual of $\alpha$ is the standard identification.

Applying the snake lemma to (2.2) gives

Corollary 2.3. Derived bundles can be calculated from the Taylor series map, $\nu^{1}: V_{X} \rightarrow \mathrm{P}^{1}(E)$ :

(1) $\operatorname{cok}(\partial)=\operatorname{cok}\left(\nu^{1}\right)$;

(2) $E_{1}=\left(\operatorname{cok}\left(\nu^{1}\right) \otimes \Omega_{X / k}^{-1}\right) /$ torsion;

(3) $\operatorname{tor}_{1}(\phi)$ is the torsion subsheaf of $\operatorname{cok}\left(\nu^{1}\right)$;

(4) $\operatorname{drk}_{1}(\phi)=\operatorname{rk}\left(\operatorname{im} \nu^{1}\right)-\operatorname{rk} E$.

Hence, the first torsion sheaf of $\phi$ measures where $\nu^{1}$ drops rank.

Remark 2.4. The map $\delta: S \rightarrow \Omega_{G / k} \otimes Q$ introduced in the proof of Proposition 2.1 is called the second fundamental form homomorphism. It differs by a factor of -1 from the second fundamental form of [AK]. As part of the proof of Proposition 2.1, we showed that $\partial$ may described as the pullback via $f$ of the second fundamental form on $G$, composed with $d f \otimes 1$ where $d f: f^{*} \Omega_{G / k} \rightarrow$ $\Omega_{X / k}$ is the cotangent map.

Local description of $\partial$. We now use Proposition 2.1 to give a description of $\partial$ in local coordinates. Looking locally, we may assume $X=\operatorname{Spec} A$ and identify $V_{X}$ with $A^{\oplus n}$ (choosing a basis for $V$ ), $E$ with $A^{\oplus r}$, and $S_{E}$ with $A^{\oplus n-r}$. The map $\phi$ becomes the matrix $M=\left(a_{i j}\right)$ whose rows will be denoted by $v_{i}$ for $i=1, \ldots, r$. Let $L=\left(b_{i j}\right)$ be the inclusion $S_{E} \rightarrow V_{X}$ and denote its columns by $w_{i}$ for $i=1, \ldots, n-r$. Recall the standard derivation, $d: A \rightarrow \Omega_{A / k}$. We may assume that $\Omega_{A / k}$ is trivial with generator $d z$. For $a \in A$, define $a^{\prime}$ by the equation

$$
d a=a^{\prime} d z
$$

Define $v_{i}^{\prime}=\left(a_{i 1}^{\prime}, \ldots, a_{i n}^{\prime}\right)$. Finally, identifying $\mathrm{P}^{1}(E)$ with $\left(A^{\oplus r}\right)^{\oplus 2} \cong A^{\oplus 2 r}$ 
as in (A.4), diagram (2.2) becomes

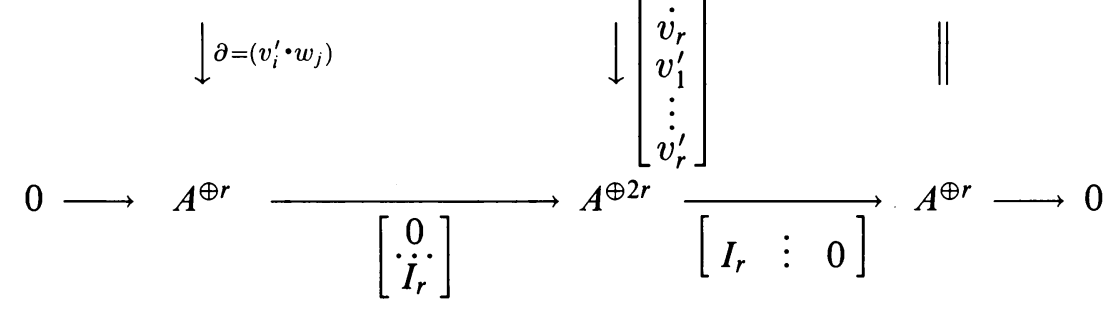

where $I_{r}$ is the $r \times r$ identity matrix. For details, see (A.4) and (A.6.4.3). Since $v_{i} \cdot w_{j}=0$, the following "dual" description comes from the product rule:

$$
\partial=\left(v_{i}^{\prime} \cdot w_{j}\right)=\left(-v_{i} \cdot w_{j}^{\prime}\right)
$$

where $w_{j}^{\prime}=\left(b_{1 j}^{\prime}, \ldots, b_{n j}^{\prime}\right)$.

For a "more local" description of $\partial$, take $A$ to be the local ring at some point $x \in X$ with local parameter $z$. The completion of $A$ is then isomorphic to the power series ring $k[[z]]$, and the inclusion of $A$ into its completion allows us to view the $v_{i}$ 's and $w_{j}$ 's as functions of $z$. The derivatives we must take are then just ordinary derivatives of power series. Finally, looking in the fiber at $x$, we get a nice interpretation of $\partial$. Consider the parametrized family of $(n-r)$-dimensional subspaces of $V$ :

$$
\Lambda(z)=\operatorname{span}\left\{w_{1}(z), \ldots, w_{n-r}(z)\right\} .
$$

In the fiber at $x$,

$$
\begin{aligned}
\partial: \Lambda(0) & \rightarrow V / \Lambda(0), \\
\sum \alpha_{i} w_{i}(0) & \mapsto \sum \alpha_{i} w_{i}^{\prime}(0) .
\end{aligned}
$$

Dually, using (2.6), take the parametrized family of $r$-dimensional subspaces of $V^{*}$

$$
\Upsilon(z)=\operatorname{span}\left\{v_{1}(z), \ldots, v_{r}(z)\right\}
$$

and in the fiber at $x$,

$$
\begin{aligned}
\partial: \Upsilon(0) & \rightarrow V^{*} / \Upsilon(0), \\
\sum \alpha_{i} v_{i}(0) & \mapsto \sum \alpha_{i} v_{i}^{\prime}(0) .
\end{aligned}
$$

This agrees with $[\mathrm{GH} 2$, p. 384, 2.1]. At most points, the rank of this map is the differential rank of $\phi$. At special points, the rank may drop, and this is measured by the torsion sheaf.

\section{FUNCTORIAL PROPERTIES OF DERIVED BUNDLES}

We consider two types of functorial properties of derived bundles: one coming from maps between bundles, and the other from maps between curves. As an application, the latter can be used to show that the associated maps, (B.4), are birational. 
Proposition 3.1. Let $V, W$ be $k$-vector spaces and $E, F$ be vector bundles on $X$. Suppose there is a commutative diagram

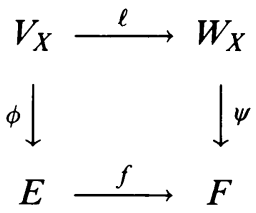

where the vertical maps are surjective. Then there are maps between derived bundles $f_{i}: E_{i} \rightarrow F_{i}$ such that

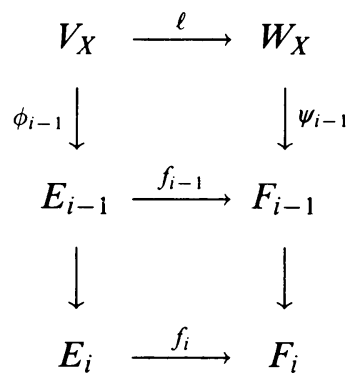

commutes for $i \geq 1$. (The vertical maps are the natural ones. Define $E_{0}=E$, $F_{0}=F, f_{0}=f, \phi_{0}=\phi$ and $\psi_{0}=\psi$.) There are also maps between torsion sheaves:

$$
g_{i}: \operatorname{tor}_{i}(\phi) \rightarrow \operatorname{tor}_{i}(\psi)
$$

(1) If $f$ is surjective, so are the $f_{i}$. In this case, for all $i \geq 1$,

$$
\mathrm{rk} E-\operatorname{rk} F \geq \sum_{j=1}^{i}\left(\operatorname{drk}_{j}(\phi)-\operatorname{drk}_{j}(\psi)\right) \text {. }
$$

(2) If $f$ is an isomorphism and $\ell$ is surjective, then the $f_{i}$ and $g_{i}$ are isomorphisms.

Proof. The proof is a straightforward diagram-chase using (2.2) and the functoriality of principal parts bundles and Taylor series maps (Appendix A).

Corollary 3.1.1. With the notation of Proposition 3.1, if $\operatorname{drk}_{1} \phi=\operatorname{rk} E$ and $f$ is surjective, then $\mathrm{drk}_{1} \psi=\mathrm{rk} F$.

Proof. This follows immediately from (1). The hypotheses imply that $E_{1}=0$ and $f_{1}$ is surjective. Thus, $F_{1}=0$ and the result follows.

Proposition 3.2. Let $Y$ be a nonsingular projective curve over $k, E$ a bundle on $Y$, and $\phi_{Y}: V_{Y} \rightarrow E$ any surjection with $V$ a $k$-vector space, as usual. Let $f: X \rightarrow Y$ be a finite, separable morphism with $X$ a nonsingular projective curve over $k$. Pulling $\phi_{Y}$ back via $f$ gives $\phi_{X}: V_{X} \rightarrow f^{*} E$, and we may consider its derived bundles $\left(f^{*} E\right)_{i}$.

(1) $f^{*}\left(E_{i}\right) \cong\left(f^{*} E\right)_{i} \quad$ (as quotients of $\left.V_{X}\right)$;

(2) length $\left(\operatorname{tor}_{i}\left(\phi_{X}\right)\right)=$ length $\left(f^{*} \operatorname{tor}_{i}\left(\phi_{Y}\right)\right)+\operatorname{drk}_{i}\left(\phi_{Y}\right)$ length $\left(\Omega_{X / Y}\right)$.

Proof. Since $E_{i}=\left(E_{i-1}\right)_{1}$, it suffices to show (1) for the case $i=1$. We can show (1) using local coordinates, but it is easier to use the duality theorem, 
(7.1). On $Y$, there is the exact sequence, (1.4),

$$
0 \rightarrow S_{E} \rightarrow V_{Y} \rightarrow E \rightarrow 0 .
$$

Consider diagram (2.2) for the dual of this sequence:

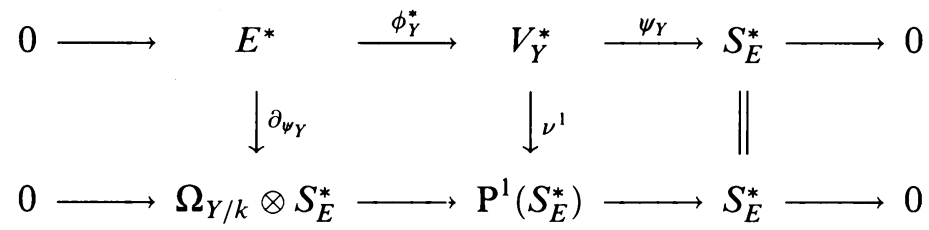

Corollary 7.1.3 of the duality theorem says that

$$
\operatorname{ker} \partial_{\psi_{Y}}=\left(E_{1}\right)^{*} \text {. }
$$

Pulling back $(*)$ to $X$ gives the exact sequence

$$
0 \longrightarrow f^{*} S_{E} \rightarrow V_{X} \rightarrow f^{*} E \rightarrow 0 .
$$

Consider (2.2) for the dual of this sequence:

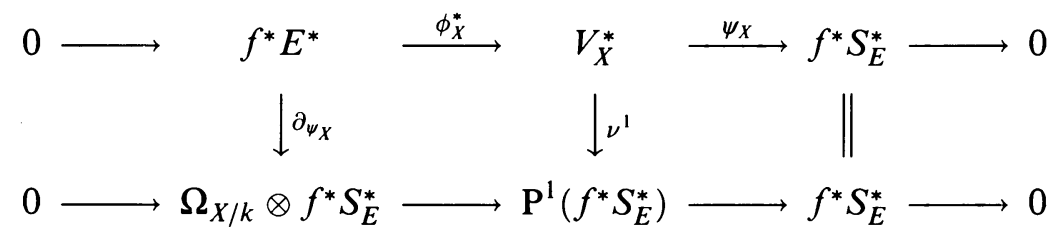

Corollary 7.1 .3 says that

$$
\operatorname{ker} \partial_{\psi_{X}}=\left(\left(f^{*} E\right)_{1}\right)^{*} \text {. }
$$

Thus, we need to show that $\left(f^{*} \operatorname{ker} \partial_{\psi_{Y}}\right)^{*} \cong\left(\operatorname{ker} \partial_{\psi_{X}}\right)^{*}$ as quotients of $V_{X}$.

There is a natural map, $f^{*} \mathrm{P}^{1}\left(S_{E}^{*}\right) \rightarrow \mathrm{P}^{1}\left(f^{*} S_{E}^{*}\right),($ A.2.4). By Proposition A.3.4 and (A.6.3.5), it induces a map from the pullback of (**) via $f$ to $(\ddagger)$. In particular, there is a commutative diagram:

$$
\begin{array}{cc}
f^{*} \operatorname{ker} \partial_{\psi_{Y}} & \longrightarrow f^{*} E^{*} \stackrel{f^{*} \partial_{\psi_{Y}}}{\longrightarrow} f^{*} \Omega_{Y / k} \otimes f^{*} S_{E}^{*} \\
\downarrow & \downarrow d f \otimes 1 \\
\operatorname{ker} \partial_{\psi_{X}} \longrightarrow f^{*} E^{*} \underset{\partial_{\psi_{X}}}{\longrightarrow} & \Omega_{X / k} \otimes f^{*} S_{E}^{*}
\end{array}
$$

Since $f$ is separable, the cotangent map $d f: f^{*} \Omega_{Y / k} \rightarrow \Omega_{X / k}$ is injective, ([H, p. 300]). Further, $f$ is flat, $\left(\left[H\right.\right.$, p. 299]), hence $f^{*} \operatorname{ker} \partial_{\psi_{Y}}=\operatorname{ker} f^{*} \partial_{\psi_{Y}}$. Therefore, it follows from the snake lemma that the leftmost vertical map is an isomorphism compatible with the natural maps to $V_{X}^{*}$. Taking duals gives (1).

To prove (2), we proceed as in the proof of (1) but without taking duals. Again, it suffices to prove the result for $i=1$. We have diagram (2.2) and its counterpart on $X$ :

$(\circledast)$

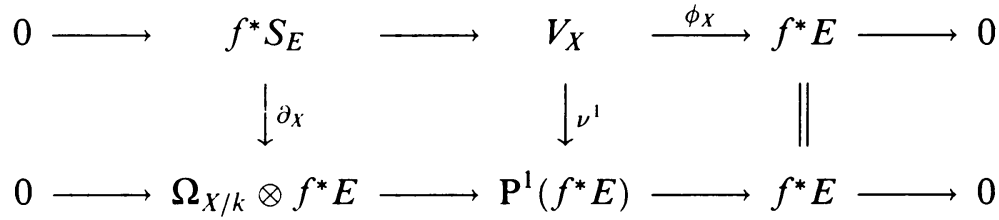


As before, (A.3.4) and (A.6.3.5) give a map of commutative diagrams (2.2) $\rightarrow$ $(\circledast)$. In particular, there is a commutative diagram:

$$
\begin{array}{cc}
f^{*} S_{E} \stackrel{f^{*} \partial_{\phi_{Y}}}{\longrightarrow} f^{*} \Omega_{Y / k} \otimes f^{*} E \\
\| \\
f^{*} S_{E} \underset{\partial_{\phi_{X}}}{\longrightarrow} & \Omega_{X / k} \otimes f^{*} E
\end{array}
$$

From this, we get the commutative diagram with exact rows

$$
\begin{aligned}
& 0 \longrightarrow f^{*} \mathrm{im} \partial_{\phi_{Y}} \longrightarrow f^{*} \Omega_{Y / k} \otimes f^{*} E \longrightarrow f^{*} \operatorname{cok} \partial_{\phi_{Y}} \longrightarrow 0 \\
& \downarrow d f \otimes 1 \\
& 0 \longrightarrow \operatorname{im} \partial_{\phi_{X}} \longrightarrow \Omega_{X / k} \otimes f^{*} E \longrightarrow 0
\end{aligned}
$$

where the left vertical map is surjective. Since $d f$ is injective with cokernel $\Omega_{X / Y}$, the snake lemma shows there is an exact sequence

$$
0 \longrightarrow f^{*} \operatorname{cok} \partial_{\phi_{X}} \longrightarrow \operatorname{cok} \partial_{\phi_{Y}} \rightarrow \Omega_{X / Y} \otimes f^{*} E \longrightarrow 0
$$

Finally, we consider the torsion sheaves in the commutative diagram with exact rows

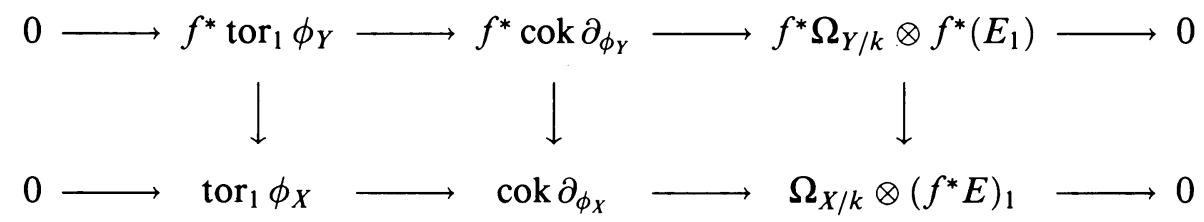

(2) follows by applying the snake lemma to this diagram and taking degrees.

Example 3.2.1. If $E=\mathrm{G}^{t}(\mathscr{L})$ for a line bundle $\mathscr{L}$ as in (B.2), then Proposition 3.2 and Theorem 8.1.1 recover Proposition B.3.6 which shows how the inflectional behavior for curves in projective space changes under covering maps.

3.3. Birationality of associated maps. Let $f: X \rightarrow \mathbb{P}(V)$ be a map of a smooth projective curve, birational to its image. Recall the $t$-th associated map,

$$
\begin{aligned}
f_{t}: X & \rightarrow G_{t} \mathbb{P}(V) \\
x & \mapsto \operatorname{Osc}_{x}^{t}(f)
\end{aligned}
$$

sending a point to its osculating space of order $t$, (for definitions, cf. B.4).

Proposition 3.3.1. Suppose that the image of $f$ is not contained in a hyperplane. Let $t<m$, and assume that the characteristic of $k$ is zero or greater than $t$ and the degree of the $t$-th osculating bundle for $f,($ B.2). Then the $t$-th associated map, $f_{t}$, is birational to its image.

Proof. Let $f$ be determined by the surjection

$$
\phi: V_{X} \rightarrow \mathscr{L} \text {. }
$$

The $t$-th associated map corresponds to the surjection

$$
\mu^{t}: V_{X} \rightarrow \mathrm{G}^{t}(\mathscr{L})
$$


(cf. B.4.2.4). The idea of the proof is that the $t$-th derived bundle of $\mu^{t}$ turns out to be $\mathscr{L}$. Thus, we can recover $f$ from $f_{t}$.

At least $f_{t}$ is not constant, for otherwise $f(X)$ would lie in a linear space of dimension $t<m$, which contradicts the definition of $m$. Factor $f_{t}$ as

$$
X \stackrel{g}{\longrightarrow} Y \stackrel{h}{\longrightarrow} G_{t}
$$

where $Y$ is the normalization of $f_{t}(X)$. Since $f_{t}$ is not constant, $g$ is finite, and $\operatorname{deg} g \leq \operatorname{deg} G^{t}(\mathscr{L})$. Hence, with our assumption on the characteristic, $g$ is also separable; we want to show that it is an isomorphism. The map $h$ corresponds to a surjection

$$
V_{Y} \rightarrow E
$$

which pulls back to $(*)$ on $X$. In Proposition 6.3.1, we will show that the $t$-th derived bundle of $\mathrm{G}^{t}(\mathscr{L})$ is $\mathscr{L}$. Hence, by Proposition 3.2

$$
\mathscr{L}=\left(\mathrm{G}^{t}(\mathscr{L})\right)_{t} \cong g^{*}\left(E_{t}\right)
$$

as quotients of $V_{X}$. Therefore, the natural map $V_{Y} \rightarrow E_{t}$ determines a map $\tilde{h}$ of $Y$ into projective space factoring $f$ :

$$
X \stackrel{g}{\longrightarrow} Y \stackrel{\tilde{h}}{\longrightarrow} \mathbb{P}(V) .
$$

Since $f$ is birational to its image, $\operatorname{deg} g=1$. In other words, $X=Y$, as desired.

\section{Characterization of DeRived bundles Via OsCulating BUNDles}

This section presents a main result of the paper, Theorem 4.2. It states that the sequence of derived bundles

$$
V_{X} \stackrel{\phi}{\longrightarrow} E \rightarrow E_{1} \rightarrow E_{2} \rightarrow \ldots
$$

lifts through the natural maps from Piene's osculating bundles

$$
V_{X} \stackrel{\phi}{\longrightarrow} E \rightarrow \mathrm{G}^{1}\left(E_{1}\right) \rightarrow \mathrm{G}^{2}\left(E_{2}\right) \rightarrow \ldots
$$

This property is used to: characterize derived bundles in Theorem 4.6; give a geometric interpretation of the sequence of differential ranks in $\S 5$, (Theorem 5.1); and recover a result of Griffiths and Harris describing curves with differential rank one in $\S 6$, (Corollary 6.2.2). We also use Theorem 4.2 to see that the sequence of differential ranks decreases, (Corollary 4.3).

Osculating bundles. The following definition is due to Piene, [Pi1]:

Definition 4.1. The image of the Taylor series map, $\nu_{\phi}^{t}: V_{X} \rightarrow \mathrm{P}^{t}(E)$, is called the osculating bundle of order $t$ for $\phi$. We denote it by $G^{t}(\phi)$ or just $G^{t}(E)$ when $\phi$ is clear from context. (It is a bundle since it is a torsion free sheaf on a smooth curve.) It comes with a natural surjection

$$
\mu^{t}: V_{X} \rightarrow \mathrm{G}^{t}(E) \text {. }
$$

The natural surjections $\mathrm{P}^{t}(E) \rightarrow \mathrm{P}^{t-1}(E)$ induce surjections $\mathrm{G}^{t}(E) \rightarrow \mathrm{G}^{t-1}(E)$. (For the definition of the Taylor series map, cf. (A.6); for generalities about osculating bundles, cf. (A.8).) 
Locally, we think of $\phi: V_{X} \rightarrow E$ as being the one-parameter family of subspaces of $V^{*}$ spanned by the rows of $\phi$, and we think of $\mu^{t}: V_{X} \rightarrow G^{t}(E)$ as being the 1-parameter family of subspaces of $V^{*}$ spanned (at a generic point of $X$ ) by the rows of $\phi$ and their derivatives up to order $t$, (A.6.4).

The immediate connection between osculating bundles and derived bundles is clear from (2.3) which states that

$$
\operatorname{cok} \partial=\operatorname{cok}\left(\nu^{1}\right)=\operatorname{cok}\left(\mathrm{G}^{1}(E) \hookrightarrow \mathrm{P}^{1}(E)\right)
$$

and hence

$$
E_{1}=\left(\operatorname{cok}\left(\mathrm{G}^{1}(E) \hookrightarrow \mathrm{P}^{1}(E)\right) \otimes \Omega_{X / k}^{-1}\right) / \text { torsion. }
$$

Characterization of derived bundles. The next theorem will show that the map $E$. $\rightarrow E_{i}$ factors through the natural surjection $\mathrm{G}^{i}\left(E_{i}\right) \rightarrow E_{i}$. Roughly, if we think of $\phi_{i}: V_{X} \rightarrow E_{i}$ and $\phi: V_{X} \rightarrow E$ as parametrized families of subspaces of $V^{*}$ spanned by the rows of $\phi_{i}$ and $\phi$, respectively, the next theorem says that each subspace in the family $V_{X} \rightarrow E$ contains a subspace spanned by the rows of $\phi_{i}$ and their derivatives up to order $i$. Theorem 4.6 shows that this property characterizes derived bundles.

Theorem 4.2. Assume the characteristic of $k$ is 0 or greater than $i+1$. Then there are surjections $\mathrm{G}^{i}\left(E_{j}\right) \rightarrow \mathrm{G}^{i+1}\left(E_{j+1}\right)$ compatible with the natural maps from $V_{X}$ and compatible with the natural surjections to lower order osculating bundles, i.e., so that the following diagram commutes

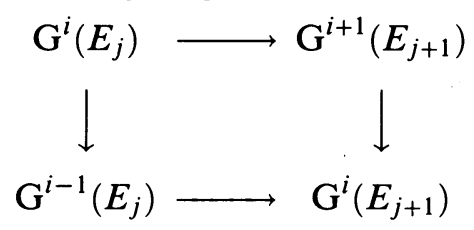

In particular, there are maps

$$
V_{X} \rightarrow E \rightarrow \mathrm{G}^{1}\left(E_{1}\right) \rightarrow \mathrm{G}^{2}\left(E_{2}\right) \rightarrow \cdots
$$

compatible with the natural surjections from $V_{X}$ and to the $E_{i}$ 's. These maps are functorial in $E,(3.1)$.

Proof. For ease of notation, we will construct the maps for $E$ and $E_{1}$, but the same argument works for $E_{j}$ and $E_{j+1}$. Let $\pi: E \rightarrow E_{1}$ be the natural surjection.

Consider the commutative diagrams, (2.2),

and
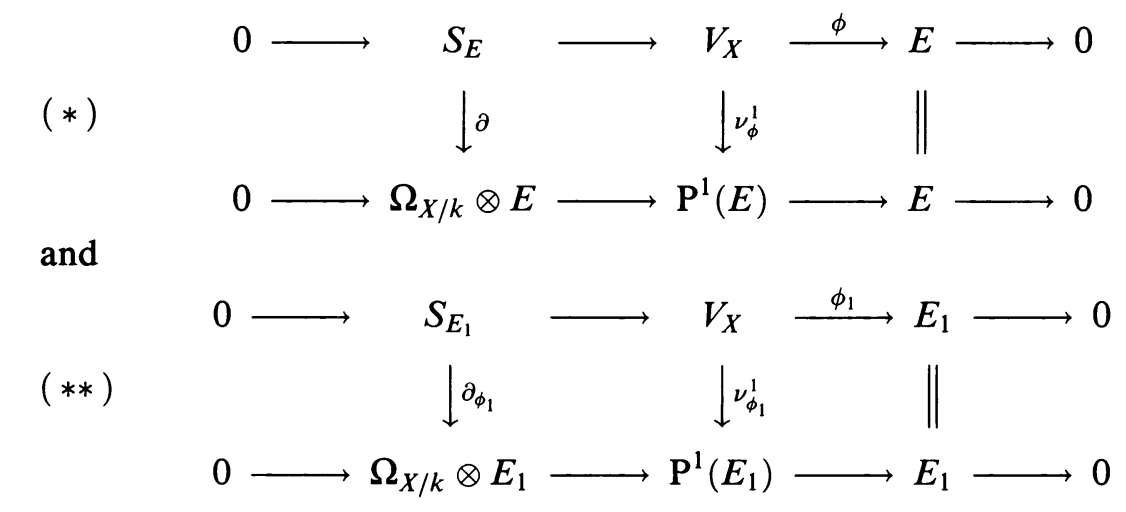
By (A.6.3.4.2), the natural map $\pi: E \rightarrow E_{1}$ induces a map of commutative diagrams $(*) \rightarrow(* *)$ which we think of as a 3-dimensional commutative diagram. As part of this diagram, we have the maps

$(* * *)$

$$
S_{E} \stackrel{\partial}{\longrightarrow} \Omega_{X / k} \otimes E \stackrel{1 \otimes \pi}{\longrightarrow} \Omega_{X / k} \otimes E_{1} .
$$

The composite is zero since, by definition of $\pi$, the natural surjection

$$
\Omega_{X / k} \otimes E \rightarrow \operatorname{cok}(\partial) / \text { torsion }=\Omega_{X / k} \otimes E_{1}
$$

is $1 \otimes \pi$.

Now consider the maps:
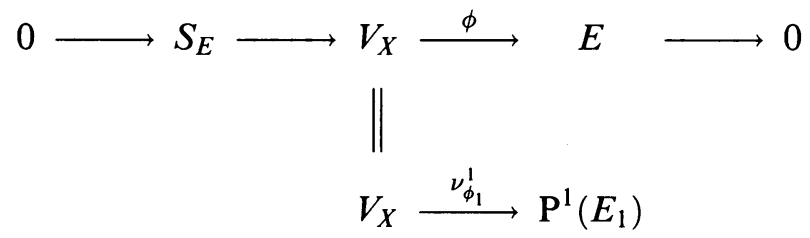

Chasing the diagram $(*) \rightarrow(* *)$ and using the fact that the composite $(* * *)$ is zero gives that the induced map $S_{E} \rightarrow \mathrm{P}^{1}\left(E_{1}\right)$ is zero. Thus there is an induced vertical map in $(\dagger), E \rightarrow \mathrm{P}^{1}\left(E_{1}\right)$. This map factors through the image of $\nu_{\phi_{1}}^{1}$ to give the surjection

$$
E \rightarrow \mathrm{G}^{1}\left(E_{1}\right)
$$

Applying the functor $G^{1}(\cdot)$ and using the isomorphism of (A.8.3) yields the surjections

$$
\mathrm{G}^{1}(E) \longrightarrow \mathrm{G}^{1}\left(\mathrm{G}^{1}\left(E_{1}\right)\right) \cong \mathrm{G}^{2}\left(E_{1}\right)
$$

Applying $G^{1}(\cdot)$ and (A.8.3) repeatedly gives the desired maps

$$
\mathrm{G}^{i}(E) \rightarrow \mathrm{G}^{i+1}\left(E_{1}\right) \text {. }
$$

The compatibility requirements follow from those in (A.8.2) and (A.8.3). Functoriality in $E$ comes from the functoriality of the maps in (*) and (**)-which was already used to construct the map $(*) \rightarrow(* *)$-and of the maps in (A.8.3). The restriction on the characteristic comes from (A.8.3).

Of course, $\operatorname{rk}\left(E_{i}\right) \geq \operatorname{rk}\left(E_{i+1}\right)$ since $E_{i} \rightarrow E_{i+1}$, but the differences in these ranks also decrease:

Corollary 4.3. The differential ranks decrease, i.e., $\operatorname{drk}_{i} \phi \geq \operatorname{drk}_{i+1} \phi$. (Note that there is no condition on the characteristic of $k$.)

Proof. Proposition 4.2 shows that $E_{i-1} \rightarrow \mathrm{G}^{1}\left(E_{i}\right)$. Thus,

$$
\begin{aligned}
\mathrm{rk} E_{i-1} & \geq \mathrm{rk} \mathrm{G}^{1}\left(E_{i}\right) \\
& =\mathrm{rkP}^{1}\left(E_{i}\right)-\mathrm{rk} E_{i+1} \\
& =2 \mathrm{rk} E_{i}-\mathrm{rk} E_{i+1} \\
\Longrightarrow \mathrm{drk}_{i} \phi=\mathrm{rk} E_{i-1}-\mathrm{rk} E_{i} & \geq \mathrm{rk} E_{i}-\mathrm{rk} E_{i+1}=\operatorname{drk}_{i+1} \phi
\end{aligned}
$$

The following proposition is a useful technical tool: 
Proposition 4.4. Consider $\mathrm{G}^{i}(E)_{1}$, the first derived bundle of $\mu^{i}: V_{X} \rightarrow \mathrm{G}^{1}(E)$.

(1) The surjection $\mathrm{G}^{i}(E) \rightarrow \mathrm{G}^{i-1}(E)$ factors through the natural map $\mathrm{G}^{i}(E)$ $\rightarrow \mathrm{G}^{i}(E)_{1}$ to give surjections

$$
\mathrm{G}^{i}(E) \rightarrow \mathrm{G}^{i}(E)_{1} \rightarrow \mathrm{G}^{i-1}(E) .
$$

These maps are compatible with the natural surjections to lower order osculating bundles; i.e., the following diagram commutes:

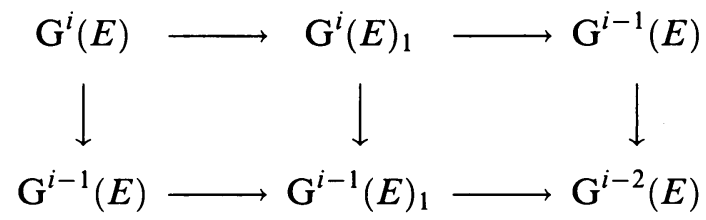

(2) $\mathrm{G}^{1}(E) \stackrel{\cong}{\rightrightarrows} \mathrm{G}^{1}\left(\mathrm{G}^{1}(E)_{1}\right)$;

(3) $\mathrm{G}^{1}\left(E_{1}\right)_{1} \cong E_{1}$.

All these maps are compatible with the surjections from $V_{X}$. (1) and (2) are functorial in $E$ and hold with $E_{j}, j \geq 0$, in place of $E$. (3) is functorial in $E_{1}$ and holds with $E_{j}, j \geq 1$, in place of $E_{1}$.

Proof. Consider the commutative diagrams, (2.2),
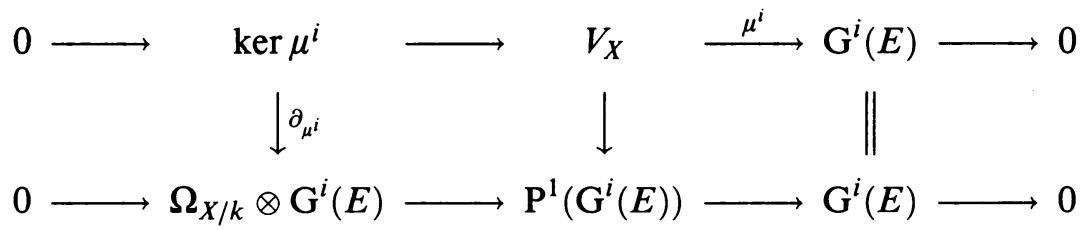

and

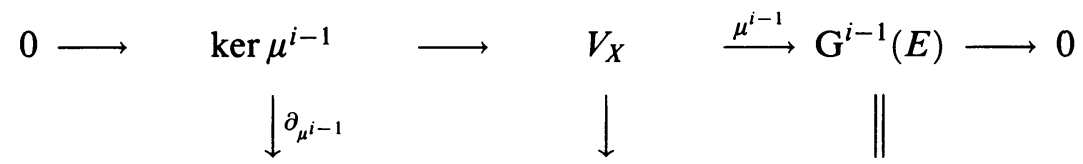

$$
0 \longrightarrow \Omega_{X / k} \otimes \mathrm{G}^{i-1}(E) \longrightarrow \mathrm{P}^{1}\left(\mathrm{G}^{i-1}(E)\right) \longrightarrow \mathrm{G}^{i-1}(E) \longrightarrow 0
$$

The natural surjection $\pi: \mathrm{G}^{i}(E) \rightarrow \mathrm{G}^{i-1}(E)$ induces a map of commutative diagrams $(*) \rightarrow(* *),($ A.6.3.4.2). In particular, there are commutative diagrams

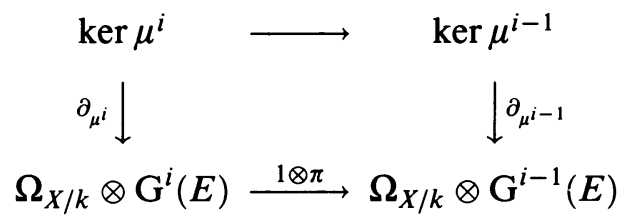

and

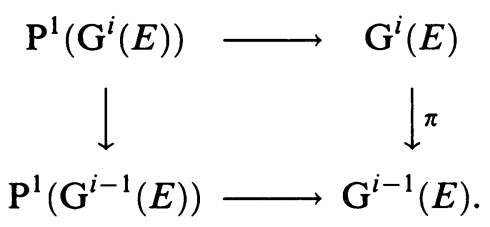


However, considering the natural maps from $V_{X}$ shows that $\pi$ factors through $\mathrm{P}^{1}\left(\mathrm{G}^{i-1}(E)\right)$ in $(\ddagger)$. Chasing the diagram $(*) \rightarrow(* *)$ then shows that $(1 \otimes \pi) \circ$ $\partial_{\mu^{i}}=0$ in (†). Therefore, there is an induced map $\operatorname{cok}\left(\partial_{\mu^{i}}\right) \rightarrow \Omega_{X / k} \otimes \mathrm{G}^{i-1}(E)$. Modding out by torsion and tensoring by $\Omega_{X / k}^{-1}$ gives

$$
\mathrm{G}^{i}(E) \rightarrow \mathrm{G}^{i}(E)_{1} \rightarrow \mathrm{G}^{i-1}(E)
$$

compatible with the natural maps from $V_{X}$. In the diagram

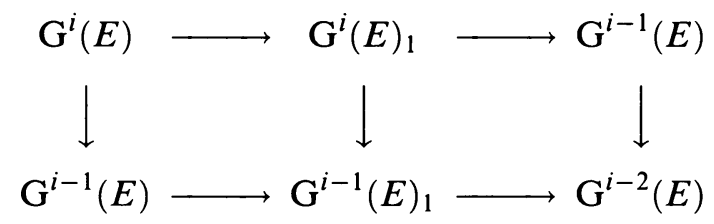

the outer square clearly commutes. The middle vertical map comes from (3.1); thus, the left square commutes. Since the horizontal arrows are surjections, this means the whole diagram commutes. This proves (1). Since $(*)$ and $(* *)$ are functorial in $E$, (A.6.3.4.2), so are the maps we have constructed.

To prove (2), apply $\mathrm{G}^{1}(\cdot)$ to (1) with $i=1$ to get $\mathrm{G}^{1}\left(\mathrm{G}^{1}(E)_{1}\right) \rightarrow \mathrm{G}^{1}(E)$. However, by Proposition 4.2 we get a map in the opposite direction: $G^{1}(E) \rightarrow$ $\mathrm{G}^{1}\left(\mathrm{G}^{1}(E)_{1}\right)$. Comparing ranks shows that the two maps must be isomorphisms. (A surjective map of bundles of the same rank must be an isomorphism.) Functoriality in $E$ follows from the corresponding property in (1) or in (4.2).

Proposition 4.2 says that $E \rightarrow \mathrm{G}^{1}\left(E_{1}\right)$. Applying Proposition 3.1 gives $E_{1} \rightarrow$ $\mathrm{G}^{1}\left(E_{1}\right)_{1}$. To prove (3), use (1) with $i=1$ and with $E_{1}$ in place of $E$ to get $G^{1}\left(E_{1}\right)_{1} \rightarrow E_{1}$. The result follows by comparing ranks as in the previous paragraph. Functoriality also follows as above.

Finally, replacing $E$ or $E_{1}$ by $E_{j}$ as in the statement of the proposition does not change the argument we have just given.

Corollary 4.5. Assume the characteristic of $k$ is zero or greater than $i$. Then $\mathrm{drk}_{1} \mu^{i-1} \geq \mathrm{drk}_{1} \mu^{i}$.

Proof. First note that by Proposition A.8.3, $\mathrm{G}^{i}(E) \cong \mathrm{G}^{1}\left(\mathrm{G}^{i-1}(E)\right)$ as quotients of $V_{X}$. Therefore, by (3.1),

$$
\mathrm{drk}_{1} \mu^{i}=\operatorname{drk}_{1} \mathrm{G}^{1}\left(\mathrm{G}^{i-1}(E)\right) .
$$

Now, replace $E$ by $\mathrm{G}^{i-1}(E)$ in (1) of Proposition 4.4 to get $\mathrm{G}^{1}\left(\mathrm{G}^{i-1}(E)\right)_{1} \rightarrow$ $\mathrm{G}^{i-1}(E)$. It follows that,

$$
\begin{aligned}
\mathrm{rkG}^{i-1}(E) & \leq \mathrm{rkG}^{1}\left(\mathrm{G}^{i-1}(E)\right)_{1} \\
& =\mathrm{rkG}^{1}\left(\mathrm{G}^{i-1}(E)\right)-\operatorname{drk}_{1} \mathrm{G}^{1}\left(\mathrm{G}^{i-1}(E)\right) \\
& =\mathrm{rkP}^{1}\left(\mathrm{G}^{i-1}(E)\right)-\mathrm{rkG}^{i-1}(E)_{1}-\operatorname{drk}_{1} \mathrm{G}^{1}\left(\mathrm{G}^{i-1}(E)\right) \\
& =\mathrm{rkP}^{1}\left(\mathrm{G}^{i-1}(E)\right)-\mathrm{rkG}^{i-1}(E)_{1}-\operatorname{drk}_{1} \mu^{i} \quad(*) \\
& =2 \mathrm{rkG}^{i-1}(E)-\mathrm{rkG}^{i-1}(E)_{1}-\operatorname{drk}_{1} \mu^{i} \\
\Longrightarrow \mathrm{drk}_{1} \mu^{i-1} & =\mathrm{rk} \mathrm{G}^{i-1}(E)-\mathrm{rkG}^{i-1}(E)_{1} \geq \operatorname{drk}_{1} \mu^{i} .
\end{aligned}
$$


Theorem 4.6 (Uniqueness of Derived Bundles). Assume the characteristic of $k$ is 0 or greater than $i$. Let $F$ be a bundle on $X$ with $\operatorname{rk} F=\operatorname{rk} E_{i}$, and let $V_{X} \rightarrow F$ be any surjection. Suppose there is a commutative diagram:

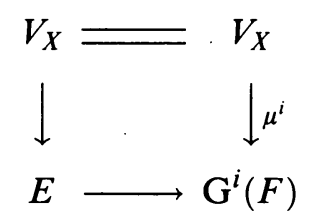

Then $F \cong E_{i}$ as quotients of $E$.

Moreover, suppose there is a string of surjections

$$
V_{X} \rightarrow F^{1} \rightarrow \cdots \rightarrow F^{i}
$$

with each $F^{j}$ a bundle on $X$ with $\mathrm{rk} F^{j}=\mathrm{rk} E_{j}$, and suppose there are commutative diagrams

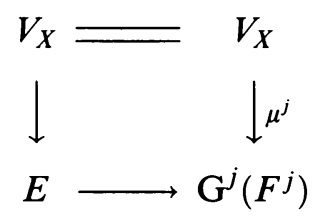

for $j=1, \ldots, i$; then the induced isomorphisms $f_{j}: F^{j} \cong E_{j}$ are compatible with the natural surjections

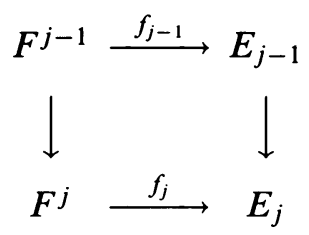

Proof. Proposition A.8.3 gives isomorphisms $\mathrm{G}^{j}(F) \cong \mathrm{G}^{1}\left(\mathrm{G}^{j-1}(F)\right)$ for $j=$ $1, \ldots, i$, (using the assumption on the characteristic of $k$ ). Combining this with Proposition 4.4, (1), yields

$$
\mathrm{G}^{j}(F)_{1}=\mathrm{G}^{1}\left(\mathrm{G}^{j-1}(F)\right)_{1} \rightarrow \mathrm{G}^{j-1}(F) .
$$

Apply this result along with Proposition 3.1, (2), repeatedly:

$$
\begin{aligned}
E & \rightarrow \mathrm{G}^{i}(F) \\
\Longrightarrow E_{i} & \rightarrow \mathrm{G}^{i}(F)_{i}=\left(\mathrm{G}^{i}(F)_{1}\right)_{i-1} \quad \text { (def. of derived bundles) } \\
& \rightarrow \mathrm{G}^{i-1}(F)_{i-1}=\left(\mathrm{G}^{i-1}(F)_{1}\right)_{i-2} \quad(*) \\
& \vdots \\
& \rightarrow F .
\end{aligned}
$$

This constructs a map $E_{i} \rightarrow F$ which must be an isomorphism since it is a surjection of bundles of the same rank.

The compatibility statement follows since the maps of $(3.1),(4.4,(1))$, and (A.8.3) respect the surjections $\mathrm{G}^{j}(F) \rightarrow \mathrm{G}^{j-1}(F)$. 


\section{GEOMETRIC INTERPRETATION OF THE SEQUENCE OF DIFFERENTIAL RANKS}

The surjections

$$
V_{X} \stackrel{\phi}{\longrightarrow} E \rightarrow \mathrm{G}^{1}\left(E_{1}\right) \rightarrow \mathrm{G}^{2}\left(E_{2}\right) \rightarrow \cdots
$$

of Theorem 4.2 suggest a way of taking local coordinates for $\phi$. Over the complex numbers, using different methods, Griffiths and Harris, [GH2], also present these local coordinates, which they call the "normal form" for a curve in a Grassmannian. We will see how this normal form is determined by the sequence of differential ranks of $\phi$ and show what is "normal" about it.

Diagram (2.2) was used to give an alternate construction of $\partial$. Recall diagram (2.5), expressing (2.2) in local coordinates on an open affine $U=\operatorname{Spec} A$ of $X$. The map $\phi: V_{X} \rightarrow E$ becomes a matrix with rows $v_{i}$ for $i=1, \ldots, r$.

Theorem 5.1 (Normal Form for a Curve in a Grassmannian). Suppose there are $\ell$ elements $u_{1}, \ldots, u_{\ell}$ of $A^{\oplus n}$ such that

$$
\left(v_{1}, \ldots, v_{r}\right)=\left(u_{1}, u_{1}^{\prime}, \ldots, u_{1}^{\left(i_{1}\right)}, \ldots, u_{\ell}, u_{\ell}^{\prime}, \ldots, u_{\ell}^{\left(i_{\ell}\right)}\right) .
$$

In other words, the rows of $\phi$ consist of the derivatives of the $u_{i}$ 's. Then

(1) $\operatorname{drk}_{1} \phi \leq \ell$;

(2) If $\operatorname{drk}_{1} \phi=\ell$, then all the higher differential ranks are determined by $i_{1}, \ldots, i_{\ell}$ :

$$
\operatorname{drk}_{m} \phi=\sharp\left\{j \mid i_{j} \geq m-1\right\}
$$

and shrinking $U$ so that it does not contain points in the support of the torsion sheaves-i.e., excluding a finite number of points-the map $\phi_{j}: V_{X} \rightarrow E_{j}$ restricted to $U$ can be expressed in local coordinates as a matrix with rows

$$
\left(u_{1}, u_{1}^{\prime}, \ldots, u_{1}^{\left(i_{1}-j\right)}, \ldots, u_{\ell}, u_{\ell}^{\prime}, \ldots, u_{\ell}^{\left(i_{\ell}-j\right)}\right)
$$

where $u_{p}^{\left(i_{p}-j\right)}$ is omitted if $i_{p}<j$. (These local forms for the $\phi_{i}$ 's are compatible with the surjections $E_{i} \rightarrow E_{i+1}$ in the natural way.)

(3) Suppose that $E_{t}=0$ for some $t$, (cf. Remark 6.1.2). Near any point not in the support of a torsion sheaf, it is possible to take coordinates as above so that $\mathrm{drk}_{1} \phi=\ell$, i.e., so that the conclusion of (2) holds.

Proof. With the $u_{i}$ 's as above, $u_{j}^{(t)} \cdot w_{i}=0$ for $t=0, \ldots, i_{j}$. So the only rows of $\partial$ that are possibly nonzero are

$$
\left(u_{j}^{\left(i_{j}+1\right)} \cdot w_{1}, \ldots, u_{j}^{\left(i_{j}+1\right)} \cdot w_{n-r}\right), \quad j=1, \ldots, \ell .
$$

Hence, $\operatorname{rk}\left(v_{i}^{\prime} \cdot w_{j}\right) \leq \ell$. This shows (1).

If $\operatorname{drk}_{1} \phi=\ell$, then the rows displayed in (*) must be $A$-linearly independent. The map $E \rightarrow E_{1}$ is defined by tensoring the composite

$$
\Omega_{X / k} \otimes E \rightarrow \operatorname{cok} \partial \longrightarrow(\operatorname{cok} \partial) / \text { torsion }=\Omega_{X / k} \otimes E_{1}
$$

by $\Omega_{X / k}^{-1}$. Thus, by shrinking $U$ if necessary to exclude the torsion of cok $\partial$, the map $E \rightarrow E_{1}$ becomes a projection

$$
A^{\oplus r} \rightarrow A^{\oplus r-\ell}
$$


onto factors of $A^{\oplus r}$ corresponding to the rows of zeros in $\partial$. Hence, there is a commutative diagram

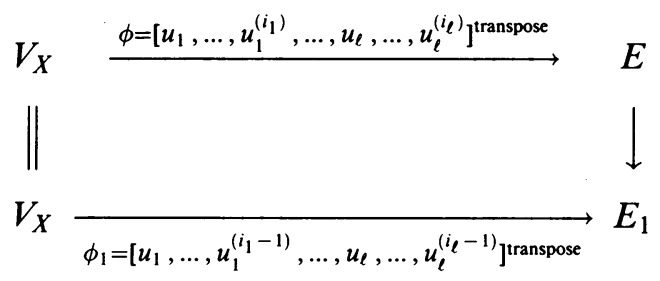

We use the convention that $u_{j}^{\left(i_{j}-1\right)}$ is omitted of $i_{j}=0$. Let $s$ be the number of $u_{i}$ 's remaining.

The preceding diagram shows the second part of (2) for $j=1$. The first part of (2) is true by supposition for $m=1$. For it to be true for $m=2$, we need to show that $\operatorname{drk}_{2} \phi=s$. We first show that the $u_{i}$ 's and their derivatives up to certain orders are linearly independent. By (2.3),

$$
\begin{aligned}
\operatorname{rkP}^{1}(E)-\operatorname{rk} E_{1} & =\operatorname{rk}\left(\operatorname{span}\left\{v_{1}, \ldots, v_{r}, v_{1}^{\prime}, \ldots, v_{r}^{\prime}\right\}\right) \\
& =\operatorname{rk}\left(\operatorname{span}\left\{u_{1}, \ldots, u_{1}^{\left(i_{1}+1\right)}, \ldots, u_{\ell}, \ldots, u_{\ell}^{\left(i_{\ell}+1\right)}\right\}\right) .
\end{aligned}
$$

But

$$
\operatorname{rkP}^{1}(E)-\operatorname{rk} E_{1}=2 \operatorname{rk} E-\left(\operatorname{rk} E-\operatorname{drk}_{1} \phi\right)=r+\ell .
$$

By counting, this implies that $u_{1}, \ldots, u_{1}^{\left(i_{1}+1\right)}, \ldots, u_{\ell}, \ldots, u_{\ell}^{\left(i_{\ell}+1\right)}$ are independent.

To ease notation, assume $i_{1}, \ldots, i_{s} \geq 1$. Use (2.3) again to get

$$
\begin{aligned}
\operatorname{rk} P^{1}\left(E_{1}\right)-\operatorname{rk} E_{2} & =\operatorname{rk}\left(\operatorname{span}\left\{u_{1}, \ldots, u_{1}^{\left(i_{1}\right)}, \ldots, u_{s}, \ldots, u_{s}^{\left(i_{s}\right)}\right\}\right) \\
& =r-\ell+s .
\end{aligned}
$$

But $\operatorname{rkP}^{1}\left(E_{1}\right)=2 \mathrm{rk} E_{1}$. Therefore,

$$
\begin{aligned}
\operatorname{drk}_{2} \phi & =\mathrm{rk} E_{1}-\mathrm{rk} E_{2}=r-\ell+s-\mathrm{rk} E_{1} \\
& =r-\ell+s-(r-\ell)=s
\end{aligned}
$$

as required.

Replacing $E=E_{0}$ by $E_{1}$ and $E_{1}$ by $E_{2}$ in the argument just given shows the first part of (2) for $m=3$ and the second part for $j=2$, and so on. Thus, (2) follows by induction.

We will prove (3) by induction on $t$ where $t$ is the smallest integer such that $E_{t+1}=0$. The case $t=0$ is true trivially. Assume the result true for $t=k-1$, and suppose $E_{k+1}$ is the last nonzero derived bundle. Let $s=\operatorname{drk}_{2} \phi=\operatorname{drk}_{1} \phi_{1}$ and $r_{1}=$ rk $E_{1}$, and apply the induction hypothesis to $\phi_{1}: V_{X} \rightarrow E_{1}$. Thus, we can choose local coordinates so that $\phi_{1}$ has the form

$$
\phi_{1}=M_{1}=\left[u_{1}, \ldots, u_{1}^{\left(i_{1}\right)}, \ldots, u_{s}, \ldots, u_{s}^{\left(i_{s}\right)}\right]^{\text {transpose }} .
$$

We will use the surjections $V_{X} \rightarrow E \rightarrow \mathrm{G}^{1}\left(E_{1}\right)$ of Theorem 4.2 to choose local coordinates for $\phi$. First, we describe $\mu^{1}: V_{X} \rightarrow \mathrm{G}^{1}\left(E_{1}\right)$ in local coordinates. By shrinking $U$, we can write $\nu^{1}: V_{X} \rightarrow \mathrm{P}^{1}\left(E_{1}\right)$ as a block matrix

$$
A^{\oplus n} \stackrel{\left[\begin{array}{l}
M_{1} \\
M_{1}^{\prime}
\end{array}\right]}{\longrightarrow} A^{\oplus 2 r_{1}}
$$


where $M_{1}^{\prime}$ is the matrix whose entries are the derivatives of those of $M_{1}$, (A.6.4.4). The bundle $G^{1}\left(E_{1}\right)$ is defined to be the image of this map. By shrinking $U$ more if necessary-to avoid the support of the torsion sheaf-we may assume $\mathrm{G}^{1}\left(E_{1}\right)$ is a subbundle of $\mathrm{P}^{1}\left(E_{1}\right)$, i.e., the quotient is a bundle. Therefore, we can take coordinates so that the rows of $\mu^{1}$ consist of those rows of $(* *)$ that are not clearly linearly dependent, namely,

$$
\left[u_{1}, \ldots, u_{1}^{\left(i_{1}+1\right)}, \ldots, u_{s}, \ldots, u_{s}^{\left(i_{s}+1\right)}\right]^{\text {transpose }} .
$$

Counting shows these rows must be linearly independent; the number of rows listed equals the rank of $\mathrm{G}^{1}\left(E_{1}\right)$ :

$$
\begin{aligned}
\mathrm{rkP}^{1}\left(E_{1}\right)-\mathrm{rkG}^{1}\left(E_{1}\right) & =\mathrm{rk} E_{2}=r_{1}-s \\
\Longrightarrow r_{1}+s & =\operatorname{rkG~}^{1}\left(E_{1}\right) .
\end{aligned}
$$

By Theorem 4.2, the surjection from $E$ to $E_{1}$ factors to give $\pi: E \rightarrow \mathrm{G}^{1}\left(E_{1}\right)$. Shrinking $U$, trivialize $E$ so that $\pi$ is just projection onto the first factors; then, locally, there is a commutative diagram

$$
\begin{array}{cc}
V_{X} \stackrel{\phi}{\longrightarrow} \underset{E}{\|} \\
V_{X} \underset{\mu^{1}}{\longrightarrow} \mathrm{G}^{1}\left(E_{1}\right)
\end{array}
$$

Therefore, $\phi$ has the form

$$
\left[u_{1}, \ldots, u_{1}^{\left(i_{1}+1\right)}, \ldots, u_{s}, \ldots, u_{s}^{\left(i_{s}+1\right)}, u_{s+1}, \ldots, u_{r-r_{1}}\right]^{\text {transpose }}
$$

for some $u_{j}, j=s+1, \ldots, r-r_{1}$. Since $r-r_{1}=\operatorname{drk}_{1} \phi, \phi$ has the desired form.

Remark 5.2. The key step of the induction argument establishing (3) of the theorem was to use the map $E \rightarrow G^{1}\left(E_{1}\right)$ of Theorem 4.2 to choose local coordinates for $E$, having already chosen them for $E_{1}$. Therefore, we regard the maps of Theorem 4.2

$$
V_{X} \stackrel{\phi}{\longrightarrow} E \rightarrow \mathrm{G}^{1}\left(E_{1}\right) \rightarrow \mathrm{G}^{2}\left(E_{2}\right) \rightarrow \ldots
$$

as the global expression of the normal form for a curve in a Grassmannian.

Example 5.3. In light of (2) of the theorem, we might say that taking derived bundles, "chops off" highest order derivatives. Suppose that the map $\phi$ has differential rank four, given in local coordinates (away from the torsion sheaf) by

$$
\phi=\left[u_{1}, u_{1}^{\prime}, u_{1}^{\prime \prime}, u_{1}^{\prime \prime \prime}, u_{2}, u_{2}^{\prime}, u_{2}^{\prime \prime}, u_{3}, u_{3}^{\prime}, u_{4}, u_{4}^{\prime}\right]^{\text {transpose }} .
$$

Then $\phi_{1}$ comes from decreasing the orders of the derivatives each by one.:

$$
\phi_{1}=\left[u_{1}, u_{1}^{\prime}, u_{1}^{\prime \prime}, u_{2}, u_{2}^{\prime}, u_{3}, u_{4}\right]^{\text {transpose }}
$$

and the second differential rank is also four. Repeat to get

$$
\phi_{2}=\left[u_{1}, u_{1}^{\prime}, u_{2}\right]^{\text {transpose }} \text {. }
$$


The third differential rank is two. Finally,

$$
\phi_{3}=\left[u_{1}\right] .
$$

The fourth differential rank is one, and all higher differential ranks are zero. If the local description of the original $\phi$ also included a constant vector, $u_{5}$, then $u_{5}$ would appear in each of the local descriptions of the $\phi_{i}$ 's; the differential ranks would not change, (6.1.2).

Example 5.4. Let $f: \mathbb{C} \rightarrow G\left(\mathbb{C}^{4}, 3\right)$ be determined by the map

$$
\phi_{z}: \mathbb{C}^{4} \stackrel{\left[\begin{array}{cccc}
1 & z & z^{2} & z^{3} \\
0 & 1 & z^{2} & z \\
0 & 0 & 1 & z
\end{array}\right]}{\longrightarrow} \mathbb{C}_{3}
$$

In other words, our map $\phi: V_{\mathbb{C}} \rightarrow E$ is a map of trivial bundles and has the above form in the fiber at $z \in \mathbb{C}$. Since the kernel of $\phi$ has rank one, the differential rank of $f$ must be one. In fact, for $z$ away from the torsion (the third torsion sheaf is supported at two points), the rows of $\phi_{z}$ span the same space as the following vector and its first two derivatives

$$
\left(3 z,-1+6 z^{2}, 1-3 z^{2}+6 z^{4}, z^{3}+3 z^{5}\right) .
$$

\section{CURVES With Differential RANK ONE}

One of the original motivations for this paper was to use Piene's osculating bundles to show that curves of differential rank one are cones over associated maps. This fact was originally observed by Griffiths and Harris, [GH2], using analytic methods. The result appears as a corollary to Theorem 6.2.1.

We then calculate the derived bundles of the osculating bundles for a curve in projective space. This calculation allows us to show that the associated maps are birational, (3.3), and to recover Piene's duality theorem for curves in projective space from our duality theorem for curves in Grassmannians, (§7).

The section begins by showing how to form cones over curves in Grassmannians. Forming a cone does not affect the differential ranks or torsion sheaves of the original curve. The map, $f$, is cone over a curve in a smaller Grassmannian if its derived bundles are not eventually zero.

6.1. Cones. Let $f: X \rightarrow G(V, r)$ and $\phi: V_{X} \rightarrow E$ be as usual, and let $W$ be a vector space over $k$ of dimension $m$. The cone over $f$ with vertex $W$ is the map

$$
\begin{aligned}
C_{W}(f): X & \rightarrow G(V \oplus W, r+m) \\
x & \mapsto f(x) \oplus W .
\end{aligned}
$$

It corresponds to the surjection

$$
C(W, \phi): V_{X} \oplus W_{X} \stackrel{\phi \oplus i d}{\longrightarrow} E \oplus W_{X}
$$

Forming a cone does not change differential ranks or torsion sheaves. 
Proposition 6.1.1. The $i$-th derived bundle of the cone, $C(W, \phi)$, is the direct sum of $W_{X}$ and the $i$-th derived bundle of the original map, $\phi$,

$$
\left(E \oplus W_{X}\right)_{i} \cong E_{i} \oplus W_{X} .
$$

These isomorphisms are compatible with the natural surjections between derived bundles. In particular, $\operatorname{drk}_{i} C(W, \phi)=\operatorname{drk}_{i} \phi$ and $\operatorname{tor}_{i} C(W, \phi)=\operatorname{tor}_{i} \phi$ for all $i$.

Proof. Since the Taylor series map and the natural surjections between principal parts bundles respect direct sums (A.1.3, A.6.3.1), diagram (2.2) becomes

$$
\begin{aligned}
& 0 \longrightarrow \quad S_{E} \oplus 0 \quad \longrightarrow \quad V_{X} \oplus W_{X} \quad \stackrel{\phi \oplus i d}{\longrightarrow} E \oplus W_{X} \longrightarrow 0 \\
& \downarrow \partial=\partial_{\phi} \oplus \partial_{i d} \quad \downarrow \nu_{\phi}^{1} \oplus \nu_{i d}^{1} \quad \| \\
& 0 \longrightarrow\left(\Omega_{X / k} \otimes E\right) \oplus\left(\Omega_{X / k} \otimes W_{X}\right) \longrightarrow \mathrm{P}^{1}(E) \oplus \mathrm{P}^{1}\left(W_{X}\right) \longrightarrow E \oplus W_{X} \longrightarrow 0
\end{aligned}
$$

Hence, $\operatorname{cok} \partial=\operatorname{cok} \partial_{\phi} \oplus \operatorname{cok} \partial_{i d}=\operatorname{cok} \partial_{\phi} \oplus\left(\Omega_{X / k} \otimes W_{X}\right)$. Therefore, the torsion of $\operatorname{cok} \partial$ is the same as the torsion of $\operatorname{cok} \partial_{\phi}$, and the first derived bundle of $C(W, \phi)$ is

$$
(\operatorname{cok} \partial / \text { torsion }) \otimes \Omega_{X / k}^{-1} \cong E_{1} \oplus W_{X}
$$

as claimed. Replacing $E$ by $E_{1}, E_{1}$ by $E_{2}$, etc., shows that the $i$-th derived bundle of $C(W, \phi)$ is as claimed. The statement about differential ranks then follows directly from the definitions

$$
\operatorname{drk}_{i} C(W, \phi)=\operatorname{rk}\left(E \oplus W_{X}\right)_{i-1}-\operatorname{rk}\left(E \oplus W_{X}\right)_{i}=\operatorname{rk} E_{i-1}-\operatorname{rk}_{i}=\operatorname{drk}_{i} \phi .
$$

The compatibility statement follows from the corresponding one for diagram (2.2) by (A.6.3.4.2).

Remark 6.1.2 (Removing Trivial Factors). Consider the sequence of derived bundles

$$
V_{X} \stackrel{\phi}{\longrightarrow} E \rightarrow E_{1} \rightarrow E_{2} \rightarrow \ldots
$$

Since these maps are surjections and $E$ has finite rank, eventually $E_{t}=E_{t+j}$ for $j \geq 0$. In this case, $\operatorname{drk}_{t+1} \phi=\operatorname{rk} E_{t}-\mathrm{rk} E_{t+1}=0$. In other words, $\operatorname{drk}_{1} \phi_{t}=0$. The next proposition will show that, with an assumption on the characteristic of $k, E_{t}$ must be trivial, and is, in fact, the largest trivial factor of $E$. The preceding proposition shows that $\phi$ is a cone over a curve in a smaller Grassmannian.

Proposition 6.1.3. If $\mathrm{drk}_{1} \phi=0$ and the characteristic of $k$ is zero or greater than $\operatorname{deg} E$, then $f: X \rightarrow G$ is a constant map and $E$ is trivial. Conversely, but with no restriction on the characteristic, if $E$ is trivial, then $\mathrm{drk}_{1} \phi=0$.

Proof. If $\mathrm{drk}_{1} \phi=0$, the tangent map $T_{X} \rightarrow f^{*} T_{G}$ is zero. Composing $f$ with the Plücker embedding, $G \rightarrow \mathbb{P}^{N}$, gives a map $X \rightarrow \mathbb{P}^{N}$ determined by

$$
\Lambda^{r} \phi: \Lambda^{r} V_{X} \rightarrow \operatorname{det} E=\mathscr{L}
$$

where $r=\operatorname{rk} E$. The tangent map of this composite is still zero, so $\operatorname{drk}_{1} \Lambda^{r} \phi=$ 0 . By Corollary 2.3, (4), the Taylor series map, $\Lambda^{r} V_{X} \rightarrow \mathrm{P}^{1}(\mathscr{L})$, is not generically surjective. Let $\operatorname{im} \Lambda^{r} V$ denote the image of the natural map $\Lambda^{r} V \rightarrow$ $\Gamma(X, \mathscr{L})$. Assuming the characteristic of $k$ is zero or greater than $\operatorname{deg} E$, 
Theorem B.2.3 says that $\operatorname{dim}\left(\operatorname{im} \Lambda^{r} V\right)<2$, i.e., $\operatorname{dim}\left(\operatorname{im} \Lambda^{r} V\right)=1$. Thus, $\mathscr{L}$ is trivial, and $f$ must be constant. Since $E$ is generated by global sections and its first Chern class, $\mathrm{c}_{1}(E)=\mathrm{c}_{1}(\mathscr{L})$, is zero, it follows that $E$ must be trivial ([F, 12.1.8]). (To see that the restriction on the characteristic is needed, consider the Frobenius map.)

On the other hand, if $E$ is trivial, then $f$ is clearly constant and $\operatorname{drk}_{1} \phi=0$. The latter assertion can be seen using local coordinates or by noting that, by (3.1), we may assume $V_{X}=E$. Thus, $S_{E}=0$ and $\operatorname{drk}_{1} \phi=0$.

6.2. Curves with differential rank one. In Theorem 4.2 we showed that the sequence of derived bundles lifts through natural maps from the osculating bundles:

$$
V_{X} \stackrel{\phi}{\longrightarrow} E \rightarrow \mathrm{G}^{1}\left(E_{1}\right) \rightarrow \mathrm{G}^{2}\left(E_{2}\right) \rightarrow \ldots
$$

In some situations we can calculate the ranks of the osculating bundles to show that these maps are isomorphisms.

Theorem 6.2.1. Let

$$
V_{X} \stackrel{\phi}{\longrightarrow} E \rightarrow E_{1} \rightarrow \cdots
$$

be the sequence of derived bundles of $\phi$. Assume

$$
\operatorname{drk}_{i} \phi= \begin{cases}d & \text { for } i=1, \ldots, m+1, \\ 0 & \text { for } i>m+1\end{cases}
$$

and assume the characteristic of $k$ is zero or greater than $\operatorname{deg} E_{m+1}$ and $m$. Then there are isomorphisms $E_{i} \cong \mathrm{G}^{m-i}\left(E_{m}\right)$ for all $i$, compatible with the natural maps from $V_{X}$ and with the natural surjections

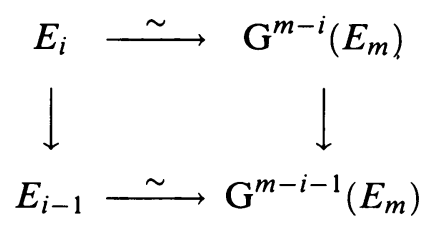

Proof. By (6.1.2), $E_{m+1}$ is trivial. By (6.1.1), we may assume $E_{m+1}=0$. (Here, for the compatibility statement, we use that the Taylor series map and the natural surjections of principal parts bundles respect direct sums, (A.6.3.1, A.2.6).

Let $i \leq m$, and consider the exact sequence

$$
V_{X} \stackrel{\nu_{\phi_{i}}^{1}}{\longrightarrow} \mathrm{P}^{1}\left(E_{i}\right) \rightarrow \operatorname{cok} \nu_{\phi_{i}}^{1} \rightarrow 0 .
$$

By Corollary 2.3, (1),

$$
\begin{aligned}
\mathrm{rk} E_{i+1} & =\mathrm{rk} \operatorname{cok} \nu_{\phi_{i}}^{1}=\mathrm{rkP}^{1}\left(E_{i}\right)-\mathrm{rk} \mathrm{G}^{1}\left(E_{i}\right) \\
& =2 \mathrm{rk} E_{i}-\mathrm{rk} \mathrm{G}^{1}\left(E_{i}\right) .
\end{aligned}
$$

But, by hypothesis, $\mathrm{rk} E_{i+1}=\mathrm{rk} E_{i}-d$. Therefore,

$$
\mathrm{rkG}^{1}\left(E_{i}\right)=\mathrm{rk} E_{i}+d=\mathrm{rk} E_{i-1} .
$$

By Theorem 4.2,

$$
E_{i-1} \rightarrow \mathrm{G}^{1}\left(E_{i}\right)
$$


This map must be an isomorphism since it is a surjection between bundles of the same rank.

The theorem follows by descending induction. For $i=m,(*)$ says $E_{m-1} \cong$ $\mathrm{G}^{1}\left(E_{m}\right)$ as required. Assume that $E_{i} \cong \mathrm{G}^{m-i}\left(E_{m}\right)$ with the desired compatiblilties. By the isomorphisms of $(*)$ and (A.8.3) we get

$$
E_{i-1} \cong \mathrm{G}^{1}\left(E_{i}\right) \cong \mathrm{G}^{1}\left(\mathrm{G}^{m-i}\left(E_{m}\right)\right) \cong \mathrm{G}^{m-i+1}\left(E_{m}\right) \text {. }
$$

The compatibility requirements follow from those of (4.2) and (A.8.3). The restriction on the characteristic is used to show that $E_{m+1}$ is trivial and to invoke (A.8.3).

The following corollary recovers a result of Griffiths and Harris, [GH2, p. 386]. If $g: X \rightarrow \mathbb{P}(V)$ is a map of a curve into projective space, recall that the $t$-the associated map

$$
\begin{aligned}
g_{t}: X & \rightarrow G_{t} \mathbb{P}(V) \\
x & \mapsto \operatorname{Osc}_{x}^{t}(g)
\end{aligned}
$$

sends a point to its osculating space of order $t$, (B.4). If $g$ corresponds to a surjection $V_{X} \rightarrow \mathscr{L}$ for some line bundle on $X$, Piene has shown that the $t$-th associated map corresponds to a surjection

$$
V_{X} \rightarrow \mathrm{G}^{t}(\mathscr{L})
$$

(cf. B.4.2.4).

Corollary 6.2.2. Assume that the characteristic of $k$ is zero or sufficiently large (as specified in the proof, below). If $\mathrm{drk}_{1} E=1$, then $E \cong \mathrm{G}^{m}(\mathscr{L}) \oplus W_{X}$ where $\mathscr{L}$ is a line bundle quotient of $E$ and $W$ a quotient of $V$. In other words, $f: X \rightarrow G$ is a cone over an associated map.

Proof. By Corollary 4.3, $\operatorname{drk}_{i-1} \phi \geq \operatorname{drk}_{i} \phi$. Therefore,

$$
\operatorname{drk}_{i} \phi= \begin{cases}1 & \text { for } i=1, \ldots, m+1, \\ 0 & \text { for } i>m+1\end{cases}
$$

for some $m$. Assume the characteristic of $k$ is zero or greater than $\operatorname{deg} E_{m+1}$ and $m$. As at the beginning of the proof of the Theorem 6.2.1, we use (6.1.2) to conclude $E_{m+1}$ is trivial and use (6.1.1) to reduce to the case where $E_{m+1}=0$. The result follows from Theorem 6.2.1 with $d=1$ and $\mathscr{L}=E_{m}$.

Remark 6.2.3. The get a geometric interpretation of Theorem 6.2.1, think of $\phi_{m}: V_{X} \rightarrow E_{m}$ as being the 1-parameter family of subspaces of the dual space $V^{*}$ locally spanned by the rows of $\phi_{m}$; then for $i<m$, Theorem 6.2.1 says that $\phi_{i}$ corresponds (generically) to the 1-parameter family of subspaces of $V^{*}$ spanned by the rows of $\phi_{m}$ and their derivatives up to order $m-i$. If $E_{m}$ is a line bundle, we are taking the derivatives of just one vector, which we think of as tracing out a curve in projective space. The space spanned by the vector and its derivatives is an osculating space for the curve.

6.3. Derived bundles of associated maps. The next proposition is, roughly, the converse to Corollary 6.2.2. It calculates the derived bundles of the osculating bundles of a curve in projective space. 
Proposition 6.3.1. Let $\mathscr{L}$ be a line bundle, and let $V \rightarrow \Gamma(X, \mathscr{L})$ be a map of vector spaces with image an $(m+1)$-dimensional subspace of generating sections. Assume that the characteristic of $k$ is zero or that $X$ is projective and the characteristic of $k$ is greater than $\operatorname{deg} \mathscr{L}$ and $m$. Then the $i$-th derived bundle of $\mu^{m}: V_{X} \rightarrow \mathrm{G}^{m}(\mathscr{L})$ is $\mathrm{G}^{m-i}(\mathscr{L})$

$$
\mathrm{G}^{m}(\mathscr{L})_{i}=\mathrm{G}^{m-i}(\mathscr{L})
$$

and $\operatorname{drk}_{i} \mu^{m}=1$ for $i=1, \ldots, m+1$. (Define $\mathrm{G}^{-1}(\mathscr{L})=0$.)

Proof. By the uniqueness theorem, (4.6), it suffices to show that for each $i$,

$$
\mathrm{G}^{m}(\mathscr{L}) \rightarrow \mathrm{G}^{i}\left(\mathrm{G}^{m-i}(\mathscr{L})\right)
$$

and that

$$
\operatorname{rkG}^{m-i}(\mathscr{L})=\operatorname{rkG}^{m}(\mathscr{L})_{i} .
$$

Proposition A.8.3 says that $\mathrm{G}^{1}\left(\mathrm{G}^{j-1}(\mathscr{L})\right) \cong \mathrm{G}^{j}(\mathscr{L})$ if the characteristic of $k$ is zero or greater than $j$. It follows immediately by induction that in our case,

$$
\mathrm{G}^{i}\left(\mathrm{G}^{m-i}(\mathscr{L})\right) \cong \mathrm{G}^{m}(\mathscr{L}) \text {. }
$$

This shows $(*)$.

The proposition now follows by induction. By Theorem B.2.3, $\mathrm{rk} \mathrm{G}^{i}(\mathscr{L})=$ $i+1$ for $i=0, \ldots, m+1$, (cf. B.2.3.3). Assume that we have shown (**) for $i \leq k-1$ so that $\mathrm{G}^{m}(\mathscr{L})_{i}=\mathrm{G}^{m-i}(\mathscr{L})$ for $i \leq k-1$; this is at least true for $k=1$. By (2.3),

$$
\begin{aligned}
\operatorname{rk~G}^{m}(\mathscr{L})_{k} & =\operatorname{rkP}^{1}\left(G^{m}(\mathscr{L})_{k-1}\right)-\operatorname{rkim} \nu^{1}\left(\mu_{k-1}^{m}\right) \\
& =\operatorname{rkP}^{1}\left(G^{m}(\mathscr{L})_{k-1}\right)-\operatorname{rk~G}^{1}\left(G^{m}(\mathscr{L})_{k-1}\right) \\
& =\operatorname{rkP}^{1}\left(G^{m-k+1}(\mathscr{L})\right)-\operatorname{rk~G}^{1}\left(G^{m-k+1}(\mathscr{L})\right) \\
& =\operatorname{rkP}^{1}\left(\mathrm{G}^{m-k+1}(\mathscr{L})\right)-\operatorname{rk~G}^{m-k+2}(\mathscr{L}) \\
& =2(m-k+2)-(m-k+3) \\
& =m-k+1=\operatorname{rk~G}^{m-k}(\mathscr{L}) .
\end{aligned}
$$

Hence $(* *)$ holds for $i=k$ as well, and $\mathrm{G}^{m}(\mathscr{L})_{k}=\mathrm{G}^{m-k}(\mathscr{L})$.

\section{Duality}

This section presents a main result of the paper: the duality theorem for curves in Grassmannians. It answers two natural questions. First, from the exact sequence of (1.4)

$$
0 \rightarrow S_{E} \rightarrow V_{X} \stackrel{\phi}{\longrightarrow} E \rightarrow 0
$$

we get the surjection

$$
\phi^{\perp}: V_{X}^{*} \rightarrow S_{E}^{*} .
$$

What are its derived bundles and osculating bundles? Second, what are the kernels of the natural maps to the derived bundles and osculating bundles of $\phi$

$$
\phi_{i}: V_{X} \rightarrow E_{i}, \quad \mu^{i}: V_{X} \rightarrow \mathrm{G}^{i}(E) .
$$


If $E$ is the osculating bundle for a curve in projective space, the answer to the second question is exactly Piene's duality theorem for curves in projective space, [Pi1]. To prove our duality theorem, we adapt Piene's proof, which simplifies in the more general context.

After proving the duality theorem, we recover Piene's theorem, explaining its connection to the classical duality theorems of the nineteenth century. We then consider the special case of a plane projective curve in order to highlight the fact that these duality theorems are fundamentally an expression of the product rule of ordinary calculus, (cf. [K2]).

Theorem 7.1. Take the dual of the exact sequence $0 \rightarrow S_{E} \rightarrow V_{X} \stackrel{\phi}{\longrightarrow} E \rightarrow 0$ to get

$$
\phi^{\perp}: V_{X}^{*} \rightarrow S_{E}^{*}
$$

with its first derived bundle, $\left(S_{E}^{*}\right)_{1}$, and first osculating bundle, $\mathrm{G}^{1}\left(S_{E}^{*}\right)$. Then

$$
\operatorname{ker}\left(\mu_{\phi}^{1}: V_{X} \rightarrow \mathrm{G}^{1}(E)\right)=\left(\left(S_{E}^{*}\right)_{1}\right)^{*}
$$

and

$$
\operatorname{ker}\left(\phi_{1}: V_{X} \rightarrow E_{1}\right)=\mathrm{G}^{1}\left(S_{E}^{*}\right)^{*} .
$$

Hence, there is a commutative diagram with exact rows:

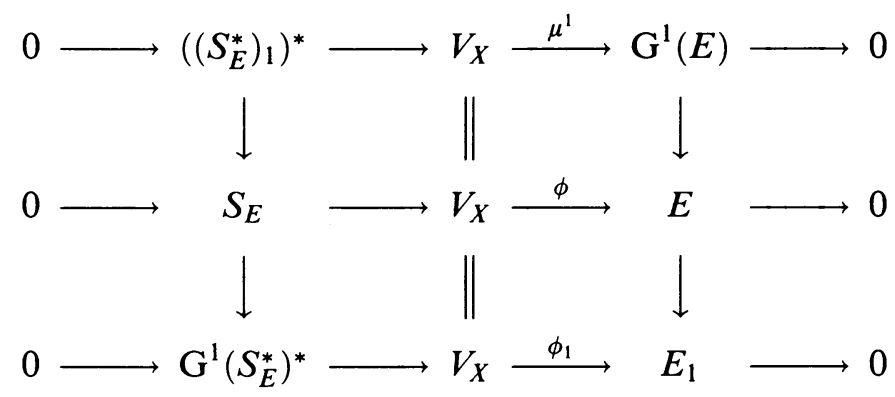

Proof. It suffices to establish the bottom rectangle of the diagram: the top then follows by replacing $E$ by $S_{E}^{*}$ and taking duals. Let $K$ denote the dual of the kernel of $V_{X}^{*} \rightarrow \mathrm{G}^{1}\left(S_{E}^{*}\right)$. The natural surjection $\mathrm{G}^{1}\left(S_{E}^{*}\right) \rightarrow S_{E}^{*}$ induces a commutative diagram

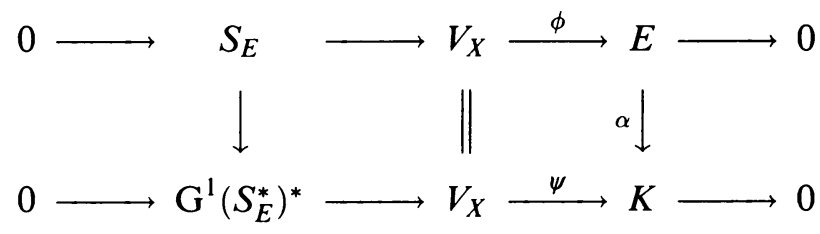

where $\psi$ and $\alpha$ are the natural maps. We will first show that $\alpha$ factors through the surjection $E \rightarrow E_{1}$ (as quotients of $V_{X}$ ). The key step-once we set up the appropriate diagrams and take local coordinates-is just the the product rule of ordinary calculus. The result then follows by showing that $E_{1}$ and $K$ have the same rank and, hence, are isomorphic. 
Consider the commutative diagram, (2.2),

$(*)$

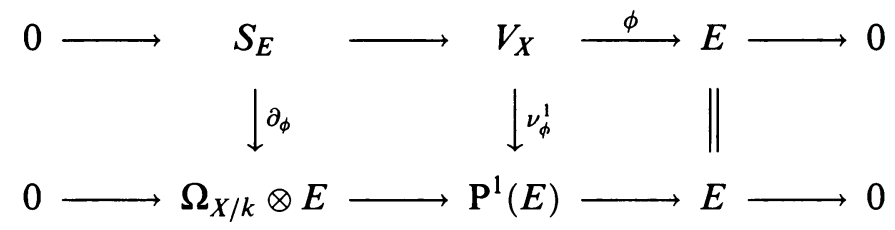

and the analogous one for $\psi$

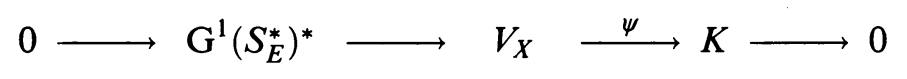

$(* *)$

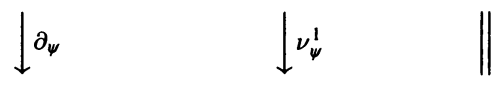

$$
0 \longrightarrow \Omega_{X / k} \otimes K \longrightarrow \mathrm{P}^{1}(K) \longrightarrow K \longrightarrow 0
$$

The map $\alpha$ induces a map of commutative diagrams $(*) \rightarrow(* *)$ by (A.6.3.4.2). In particular, we have

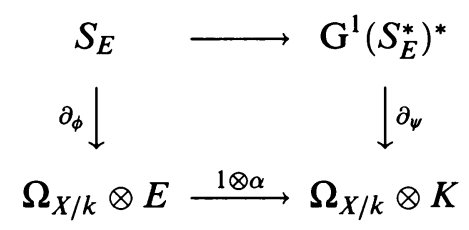

By definition, $E_{1}=\left(\operatorname{cok} \partial_{\phi} \otimes \Omega_{X / k}^{-1}\right) /$ torsion. Thus, to show that $\alpha$ factors through $E_{1}$, it suffices to show that $(1 \otimes \alpha) \circ \partial_{\phi}=0$. Chasing around $(*) \rightarrow(* *)$, it suffices to show that the following composite is zero:

$$
S_{E} \rightarrow V_{X} \stackrel{\nu_{\psi}^{1}}{\longrightarrow} \mathrm{P}^{1}(K) \text {. }
$$

We will check this using local coordinates. Consider the following commutative diagram (notation to be explained):

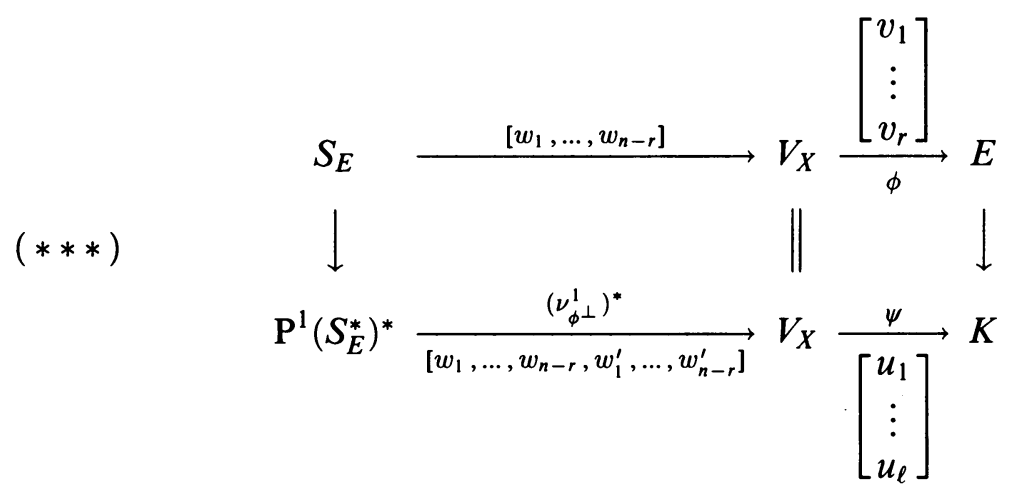

The composite

$$
S_{E} \rightarrow \mathrm{P}^{1}\left(S_{E}^{*}\right)^{*} \stackrel{\left(\nu_{\phi^{\perp}}^{1}\right)^{*}}{\longrightarrow} V_{X}
$$

is the same as the composite

$$
S_{E} \rightarrow \mathrm{G}^{1}\left(S_{E}^{*}\right)^{*} \rightarrow V_{X}
$$


of $(\dagger)$ since $\mathrm{G}^{1}\left(S_{E}^{*}\right) \rightarrow S_{E}^{*}$ factors through $\mathrm{P}^{1}\left(S_{E}^{*}\right) \rightarrow S_{E}^{*}$.

Here is the meaning of the $u_{i}$ 's, $v_{i}$ 's, and $w_{i}$ 's. Given a point $x \in X$, take an open affine set $U=\operatorname{Spec} A$ about $x$, small enough so that $E, S_{E}, K$, and $\Omega_{X / k}$ are trivial when restricted to $U$. Identifying $V_{X}$ with $A^{\oplus n}$ and $E$ with $A^{\oplus r}$ on $U$, the map $\phi$ becomes

$$
A^{\oplus n} \stackrel{\left[\begin{array}{c}
v_{1} \\
\vdots \\
v_{r}
\end{array}\right]}{\longrightarrow} A^{\oplus r}
$$

Each $v_{i}$ is a row vector: $v_{i}=\left(a_{i 1}, \ldots, a_{i n}\right)$ where $a_{i j} \in A$. The $u_{i}$ 's and $w_{i}$ 's are defined similarly (let $\ell=\operatorname{rk} K$ ), but each $w_{i}$ is a column vector. A local trivialization of $S_{E}$ determines one for $S_{E}^{*}$. This, along with a local trivialization of $\Omega_{X / k}$ determines one of $\mathrm{P}^{1}\left(S_{E}^{*}\right)$, (A.4.8), so that locally the Taylor series map is given by the transpose of $\left[w_{1}, \ldots, w_{n-r}, w_{1}^{\prime}, \ldots, w_{n-r}^{\prime}\right]$ as indicated in $(* * *),(A .6 .4 .4)$. (For the definition of the derivative $w_{i}^{\prime}$, cf. (A.4.1) and (A.6.4.2).)

With similar notation, our problem is to show that the following composite is zero:

$$
S_{E} \stackrel{-\left[w_{1}, \ldots, w_{n-r}\right]}{\longrightarrow} V_{X} \stackrel{\left[u_{1}, \ldots, u_{\ell}, u_{1}^{\prime}, \ldots, u_{\ell}^{\prime}\right]^{\text {ranspose }}}{\longrightarrow} \mathrm{P}^{1}(K) .
$$

From $(* * *)$, we know that each dot product $w_{i} \cdot u_{j}=w_{i}^{\prime} \cdot u_{j}=0$. By the product rule, it follows that $w_{i} \cdot u_{j}^{\prime}=0$ as required.

We have shown that $\alpha$ factors through $E \rightarrow E_{1}$. Hence, there is a commutative diagram

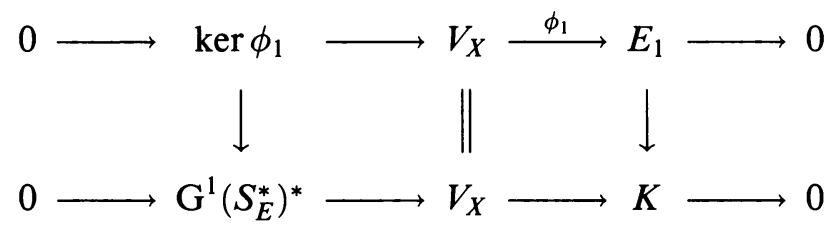

It remains to be shown that $\mathrm{rk} K=\operatorname{rk} E_{1}$. (Then $E_{1} \rightarrow K$ is an isomorphism since it's a surjective map of bundles of the same rank.) Calculate:

$$
\begin{aligned}
\operatorname{rk} K & =n-\operatorname{rk~G}^{1}\left(S_{E}^{*}\right) \\
& =n-\operatorname{rk} P^{1}\left(S_{E}^{*}\right)+\operatorname{rk}\left(S_{E}^{*}\right)_{1} \\
& =n-2 \operatorname{rk} S_{E}^{*}+\operatorname{rk}\left(S_{E}\right)_{1}^{*} \\
& =n-\operatorname{rk} S_{E}^{*}-\operatorname{drk}_{1} S_{E}^{*} \\
& =\operatorname{rk} E-\operatorname{drk}_{1} S_{E}^{*} .
\end{aligned}
$$

Thus,

$$
\begin{aligned}
& E_{1} \rightarrow K \\
& \Rightarrow \mathrm{rk} E_{1}=\mathrm{rk} E-\mathrm{drk}_{1} E \geq \mathrm{rk} K=\mathrm{rk} E-\operatorname{drk}_{1} S_{E}^{*} \\
& \Rightarrow \operatorname{drk}_{1} S_{E}^{*} \geq \operatorname{drk}_{1} E
\end{aligned}
$$

with equality if and only if $\operatorname{rk} E_{1}=\operatorname{rk} K$. To see that we get an equality here, dualize everything; consider the exact sequence

$$
0 \rightarrow E^{*} \stackrel{\phi^{*}}{\longrightarrow} V_{X}^{*} \stackrel{\phi^{\perp}}{\longrightarrow} S_{E}^{*} \rightarrow 0 .
$$


Repeating the whole argument with $\phi^{\perp}$ in place of $\phi$ gives that

$$
\operatorname{drk}_{1} E \geq \operatorname{drk}_{1} S_{E}^{*}
$$

and we are done.

The theorem can be used to calculate the kernels of the maps to the derived bundles and osculating bundles. This corollary and the one following it suggest alternative definitions of derived bundles.

Corollary 7.1.2. Let the characteristic of $k$ be zero or greater than $i$. There are exact sequences

$$
0 \rightarrow \mathrm{G}^{i}\left(S_{E}^{*}\right)^{*} \stackrel{\left(\mu_{\phi^{\perp}}^{i}\right)^{*}}{\longrightarrow} V_{X} \stackrel{\phi_{i}}{\longrightarrow} E_{i} \longrightarrow 0
$$

and dually,

$$
0 \longrightarrow\left(\left(S_{E}^{*}\right)_{i}\right)^{*} \longrightarrow V_{X} \stackrel{\mu^{i}}{\longrightarrow} \mathrm{G}^{i}(E) \longrightarrow 0
$$

Proof. Apply the duality theorem to each $E_{i}$ successively, and use Proposition A.8.3 to say that $\mathrm{G}^{1}\left(\mathrm{G}^{i-1}\left(S_{E}^{*}\right)\right) \cong \mathrm{G}^{i}\left(S_{E}^{*}\right)$.

To define the derived bundles of $\phi$, we looked at the cokernel of $\partial: S_{E} \rightarrow$ $E \otimes \Omega_{X / k}$. The next corollary shows what happens if we consider the kernel instead.

Corollary 7.1.3. $\operatorname{ker}\left(\partial: S_{E} \rightarrow E \otimes \Omega_{X / k}\right)=\left(\left(S_{E}^{*}\right)_{1}\right)^{*}=\operatorname{ker}\left(\mu^{1}: V_{X} \rightarrow \mathrm{G}^{1}(E)\right)$.

Proof. Corollary 2.3 gives $\operatorname{ker} \partial \cong \operatorname{ker} \nu^{1}=\operatorname{ker} \mu^{1}$, and then apply the theorem.

Combining Corollary 7.2.1 with Theorem 6.2.1 gives

Corollary 7.1.4. Assume that

$$
\operatorname{drk}_{i} \phi= \begin{cases}d, & \text { for } i=1, \ldots, m+1, \\ 0, & \text { for } i>m+1\end{cases}
$$

and assume the characteristic of $k$ is zero or greater than $\operatorname{deg} E_{m+1}$ and $m$. Then, for $i=1, \ldots, m$, there is an isomorphism of exact sequences

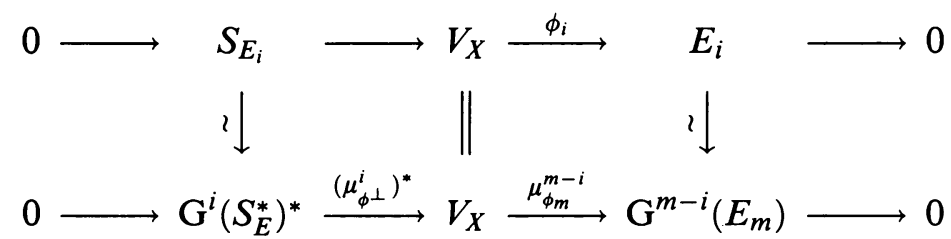

7.2. Piene duality theorem (cf. B.5). Let $V \subset \Gamma(X, \mathscr{L})$ be an $(n+1)$ dimensional vector space of generating sections of a line bundle on $X$ and $f: X \rightarrow \mathbb{P}(V)$ the corresponding map to projective space. The map of vector bundles corresponding to the $t$-th associated map of $f$ is

$$
\mu^{t}: V_{X} \rightarrow \mathrm{G}^{t}(\mathscr{L}) \text {. }
$$

Corollary 7.2.1 (Piene Duality Theorem, [Pi1]). Let $X$ be a smooth projective curve, and assume that the characteristic of $k$ is zero or greater than $n$ and the degree of $\mathscr{L}$. Let $K=\operatorname{ker} \mu^{n-1}$ with its natural map $V_{X}^{*} \rightarrow K^{*}$. Then

$$
\operatorname{ker} \mu^{i}=\mathrm{G}^{n-1-i}\left(K^{*}\right)
$$


Proof. By Proposition 6.3.1, $\mathrm{G}^{i}(\mathscr{L})$ is the $(n-i-1)$-th derived bundle of $\mathrm{G}^{n-1}(\mathscr{L})$. Therefore, the result follows from Corollary 7.1.2.

In sum, Piene's theorem is that there is a commutative diagram with exact rows:

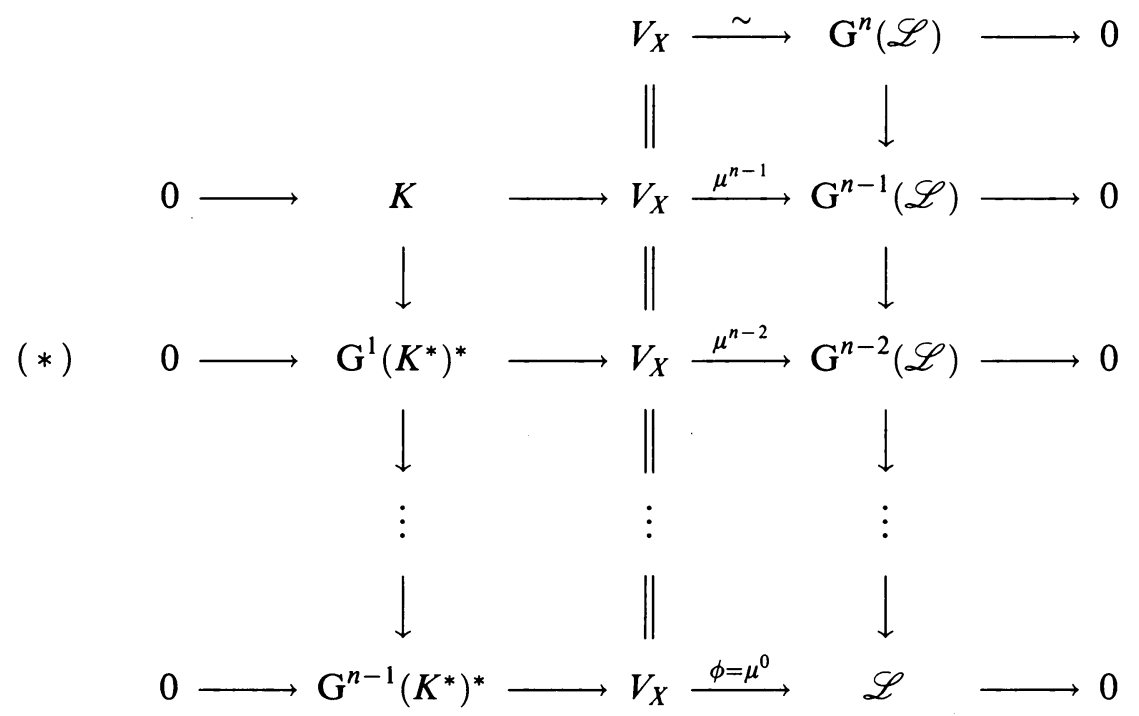

The maps on the right-hand side of this diagram correspond to the associated maps

$$
\begin{aligned}
f_{t}: X & \rightarrow G_{t} \mathbb{P}(V) \\
x & \mapsto \operatorname{Osc}_{x}^{t}
\end{aligned}
$$

sending a point to its osculating space of order $t$. The duals of the maps on the right, coming from the kernels of the $\mu^{i}$ 's, correspond to the $t$-th dual maps

$$
\begin{aligned}
f^{t}: X & \rightarrow G_{n-t-1} \mathbb{P}\left(V^{*}\right) \\
x & \mapsto H_{x}^{t}
\end{aligned}
$$

sending a point to the set of hyperplanes containing an osculating space of order $t$. The map determined by the natural surjection $V_{X}^{*} \rightarrow K^{*}$,

$$
f^{*}=f^{n-1}: X \rightarrow \mathbb{P}\left(V^{*}\right)
$$

sending a point to its osculating hyperplane, is called the dual of $f$. Diagram (*) shows that

$$
\left(f^{*}\right)^{t}=f_{n-t-1}
$$

and in particular, the double dual of $f$ is $f$, itself

$$
\left(f^{*}\right)^{*}=f .
$$

7.3. Duality theorems and the product rule. The key step in the proof of the duality theorem is just the product rule of ordinary calculus. Its role is most easily seen in the special case of a curve in the complex projective plane.

Think of the curve as being swept out by a vector, $v(z)$, in affine three-space where $z$ is a local parameter for the curve. Let $w(z)$ be a vector normal to the subspace spanned by $v(z)$ and $v^{\prime}(z)$. Then $w(z)$ sweeps out the dual curve. 
To find the dual of the dual curve, repeat this construction with $w$ in place of $v$. To see that we get back the original curve, it suffices to check that $v(z)$ is normal to the subspace spanned by $w(z)$ and $w^{\prime}(z)$. In other words, we must show that

$$
v(z) \cdot w(z)=0 \quad \text { and } \quad v(z) \cdot w^{\prime}(z)=0 .
$$

By definition of $w(z)$, we know that

$$
v(z) \cdot w(z)=0 \quad \text { and } \quad v^{\prime}(z) \cdot w(z)=0 .
$$

Therefore, the result follows from the product rule:

$$
0=(v(z) \cdot w(z))^{\prime}=v^{\prime}(z) \cdot w(z)+v(z) \cdot w^{\prime}(z)=v(z) \cdot w^{\prime}(z) .
$$

Let us now compare this rough sketch of a proof of the duality theorem for plane curves with the duality theorem presented in this section. If the curve is given by a surjection $V_{X} \rightarrow \mathscr{L}$ where $\mathscr{L}$ is a line bundle on the curve, let $K$ be the kernel of the corresponding map to the osculating bundle, $\mu^{1}: V_{X} \rightarrow \mathrm{G}^{1}(\mathscr{L})$. The duality theorem says that there is a commutative diagram with exact rows

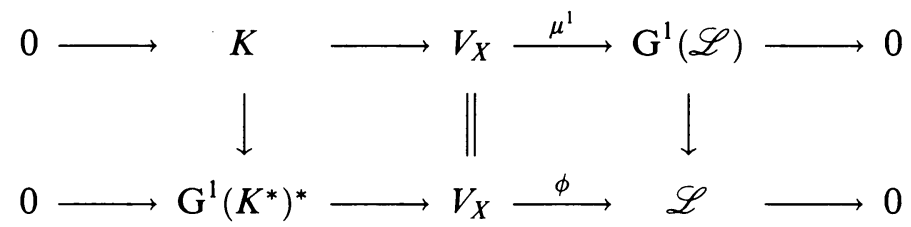

Locally, we think of $\phi$ as the vector $v(z)$. The map $\mu^{1}$ becomes a matrix with rows $v(z)$ and $v^{\prime}(z)$, (at least at a generic point). The vector $w(z)$ gives the map $V_{X}^{*} \rightarrow K^{*}$, defining the dual curve. The exactness of the bottom row of the diagram is the statement that $v$ is normal to the subspace spanned by $w$ and $w^{\prime}$.

\section{TORSION SHEAVES}

By Corollary 2.3, the torsion sheaves can be calculated using the Taylor series map $\nu^{1}: V_{X} \rightarrow \mathrm{P}^{1}(E)$ since $\operatorname{cok} \partial=\operatorname{cok} \nu^{1}$. The torsion sheaf measures where the Taylor series map drops rank. This paper focuses mainly on differential ranks; the analysis of torsion sheaves is far from complete. What is lacking is a convenient local parametrization for a curve in a Grassmannian such as that which exists in the special case of a curve in projective space (B.1). However, it is clear that torsion sheaves generalize the classical notion of stationary indices, (Theorem 8.1.1), and thus describe what might be called the inflectionary behavior of a curve in a Grassmannian. There are other ways of measuring this behavior, and we will briefly mention these. We end the section by seeing what the duality theorem says about torsion sheaves.

8.1. Stationary indices. In the special case of a curve in projective space, the lengths of the torsion sheaves are the classical stationary indices measuring how a curve flexes in space.

Let $V \subset \Gamma(X, \mathscr{L})$ where $\operatorname{dim} V=n+1$ and $\mathscr{L}$ is a line bundle on the smooth projective curve $X$. Assume the characteristic of $k$ is zero or greater than $\operatorname{deg} \mathscr{L}$ and $n$. By Theorem B.2.3, $\operatorname{rk~G}^{i}(\mathscr{L})=i+1$ for $i=0, \ldots, n$. In particular, $\mathrm{G}^{n}(\mathscr{L})=V_{X}$. We have defined the map

$$
\mu^{n-1}: V_{X} \rightarrow \mathrm{G}^{n-1}(\mathscr{L})
$$


and seen in Proposition 6.3.1 that the string of derived bundles of $\mu^{n-1}$ is

$$
V_{X} \rightarrow \mathrm{G}^{n-1}(\mathscr{L}) \rightarrow \mathrm{G}^{n-2}(\mathscr{L}) \rightarrow \ldots \rightarrow \mathscr{L} \text {. }
$$

The inflection numbers, $\alpha_{i}$, defined and discussed in Appendix B.1, satisfy the equation

$$
\operatorname{length}\left(\operatorname{cok}\left(\mathrm{G}^{i}(\mathscr{L}) \hookrightarrow \mathrm{P}^{i}(\mathscr{L})\right)\right)=\operatorname{deg} \mathrm{P}^{i}(\mathscr{L})-\sum_{j=1}^{i} \alpha_{j}
$$

for $i=1, \ldots, t$.

Theorem 8.1.1. The $i$-th torsion number for $\mu^{n-1}$ is

$$
\text { length }\left(\text { tor }_{i} \mu^{n-1}\right)=\alpha_{n-i+1}-\alpha_{n-i}
$$

the so-called $(n-i)$-th stationary index.

Proof. Let

$$
V_{X} \stackrel{\phi}{\longrightarrow} E \rightarrow E_{1} \rightarrow \ldots
$$

be the sequence of derived bundles for any map $\phi$. Letting $S_{E_{i}}$ denote the kernel of the composite, $\phi_{i}: V_{X} \rightarrow E_{i}$, there is a commutative diagram

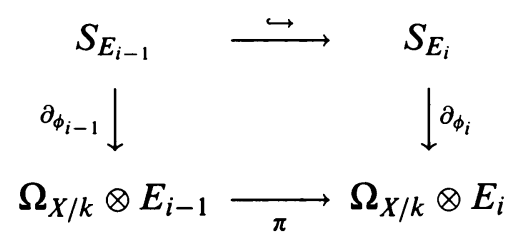

with $\pi \circ \partial_{\phi_{i-1}}=0$. This follows from the definition of derived bundles:

$$
\Omega_{X / k} \otimes E_{i} \stackrel{\text { def }}{=} \operatorname{cok} \partial_{\phi_{i-1}} / \text { torsion }
$$

Thus, there is an induced map

$$
S_{E_{i}} / S_{E_{i-1}} \stackrel{j}{\longrightarrow} \Omega_{X / k} \otimes E_{i}
$$

with cok $j /$ torsion $=\Omega_{X / k} \otimes E_{i+1}$. In our case, $E_{i}=\mathrm{G}^{n-i-1}(\mathscr{L})$, and the Piene duality theorem (7.2.1) says that $S_{E_{i}}=\mathrm{G}^{i}\left(K^{*}\right)^{*}$ where $K=\operatorname{ker} \mu^{n-1}$, a line bundle. Therefore, $(*)$ becomes

$$
\mathrm{G}^{i}\left(K^{*}\right)^{*} / \mathrm{G}^{i-1}\left(K^{*}\right)^{*} \stackrel{j}{\longrightarrow} \Omega_{X / k} \otimes \mathrm{G}^{n-i-1}(\mathscr{L})
$$

with cok $j /$ torsion $=\mathrm{G}^{n-i-2}(\mathscr{L})$. Counting ranks implies that $j$ is injective.

Consider the exact sequences

$$
0 \longrightarrow \mathrm{G}^{i}\left(K^{*}\right)^{*} / \mathrm{G}^{i-1}\left(K^{*}\right)^{*} \stackrel{j}{\longrightarrow} \Omega_{X / k} \otimes \mathrm{G}^{n-i-1}(\mathscr{L}) \rightarrow \text { cok } \rightarrow 0
$$

and

$$
0 \rightarrow \operatorname{tor}_{i+1} \mu^{n-1} \rightarrow \text { cok } \rightarrow \Omega_{X / k} \otimes \mathrm{G}^{n-i-2}(\mathscr{L}) \rightarrow 0 .
$$

Taking degrees yields

$$
\begin{aligned}
\text { length }\left(\text { tor }_{i+1} \mu^{n-1}\right)= & \operatorname{deg}(\operatorname{cok})-\operatorname{deg}\left(\Omega_{X / k} \otimes \mathrm{G}^{n-i-2}(\mathscr{L})\right) \\
= & \operatorname{deg}\left(\Omega_{X / k} \otimes \mathrm{G}^{n-i-1}(\mathscr{L})\right)-\operatorname{deg}\left(\mathrm{G}^{i}\left(K^{*}\right)^{*}\right) \\
& +\operatorname{deg}\left(\mathrm{G}^{i-1}\left(K^{*}\right)^{*}\right)-\operatorname{deg}\left(\Omega_{X / k} \otimes \mathrm{G}^{n-i-2}(\mathscr{L})\right)
\end{aligned}
$$


Now use the exact sequence

$$
0 \longrightarrow \mathrm{G}^{i}\left(K^{*}\right)^{*} \longrightarrow V_{X} \longrightarrow \mathrm{G}^{n-i-1}(\mathscr{L}) \longrightarrow 0
$$

and let $d_{i}=\operatorname{deg}\left(\mathrm{G}^{i}(\mathscr{L})\right)$ to get

$$
\begin{aligned}
\text { length }\left(\operatorname{tor}_{i+1} \mu^{n-1}\right)= & d_{n-i-1}+(n-i)(2 g-2)+d_{n-i-1} \\
& -d_{n-i}-d_{n-i-2}-(n-i-1)(2 g-2) \\
= & 2 d_{n-i-1}-d_{n-i}-d_{n-i-2}+2 g-2 \\
= & \alpha_{n-i}-\alpha_{n-i-1} \quad \text { (Plücker formula, B.3.3). }
\end{aligned}
$$

Remark 8.1.2. Let $D_{i}$ be the divisor corresponding to the $i$-th torsion sheaf. The proof of Theorem 8.1.1 can be modified to show that

$$
D_{i}=\sum_{x \in X}\left(\alpha_{n-i+1}(x)-\alpha_{n-i}(x)\right) \cdot x
$$

as divisor classes. (The Plücker formulas can also be interpreted as a statement about divisor classes.)

Example 8.2. Another special case where we can calculate the torsion numbers is when $X$ is a projective curve, $\operatorname{rk} V_{X}=2 \mathrm{rk} E$, and the differential rank of $E$ is as large as possible, namely, $r=\mathrm{rk} E$. In that case, the Taylor series map is injective and generically surjective. Therefore, the torsion number is

$$
\begin{aligned}
\operatorname{deg} \mathrm{P}^{1}(E)-\operatorname{deg} V_{X} & =\operatorname{deg} \mathrm{P}^{1}(E) \\
& =\operatorname{deg} \Omega_{X / k} \otimes E+\operatorname{deg} E \\
& =2 \operatorname{deg} E+r(2 g-2) .
\end{aligned}
$$

This calculation appears in [Cn], (also cf. [Pi2]). If this torsion number is zero, then $g=0$ and $\operatorname{deg} E=r$. So $X=\mathbb{P}^{1}$ and $E=\bigoplus_{i=1}^{r} \mathscr{O}\left(n_{i}\right)$ with $\sum n_{i}=r$. Moreover, since $V_{X} \rightarrow E$ is surjective, each $n_{i}$ is nonnegative and, in fact, positive since otherwise the first derived bundle would have a trivial factor instead of being zero, (6.1.1). Thus,

$$
E=\mathscr{O}(1)^{\oplus r}
$$

and $V=\Gamma\left(\mathbb{P}^{1}, \mathscr{O}(1)^{\oplus r}\right)$.

8.3. Other measures of inflection. Canuto $[\mathrm{Cn}]$ has suggested three measures of the inflectionary behavior of a curve in a Grassmannian. The first comes from embedding the Grassmannian in projective space by the Plücker embedding and considering the inflectionary numbers for the curve in that projective space, (B.1). A second set of invariants of the embedding of the curve are the orders of vanishing of the sections of $E$ in $\operatorname{im}(\phi: V \rightarrow \Gamma(X, E))$. The third measure comes from looking at the order of contact of the Schubert cycles with the curve at a given point. Canuto shows that these notions are not equivalent for a curve in a general Grassmannian (as they are for a curve in projective space). We have not analyzed the relation of those measures of inflection with our torsion sheaves. 
8.4. Torsion sheaves and the duality theorem. Take the dual of the exact sequence $0 \rightarrow S_{E} \rightarrow V_{X} \stackrel{\phi}{\longrightarrow} E$ to define a map

$$
\phi^{\perp}: V_{X}^{*} \longrightarrow S_{E}^{*}
$$

There are corresponding maps to the osculating bundles, $(\S 4)$,

$$
\mu_{\phi^{\perp}}^{i}: V_{X} \rightarrow \mathrm{G}^{i}\left(S_{E}^{*}\right) .
$$

We use the duality theorem, (7.1), to show

Proposition 8.4.1. Assume the characteristic of $k$ is zero or greater than $i$. Then

$$
\text { tor }_{i} \phi \cong \operatorname{tor}_{1} \mu_{\phi^{\perp}}^{i} \text {. }
$$

Proof. By (7.1.2), it suffices to show the result for the case $i=1$. Since the torsion sheaves are supported on a finite set of points, the question is local. Near any point $x \in X$, take local coodinates as in $\S 2$. so that $\partial_{\phi}$ becomes a matrix

$$
A^{\oplus n-r} \stackrel{\left(v_{i}^{\prime} \cdot w_{j}\right)}{\longrightarrow} A^{\oplus r} .
$$

Using dual cordinates, the map $\partial_{\phi^{\perp}}$ may be written

$$
A^{\oplus r} \stackrel{\left(w_{i}^{\prime} \cdot v_{j}\right)}{\longrightarrow} A^{\oplus n-r} .
$$

However, as in (2.6), since $v_{i} \cdot w_{j}=0$, the product rule says that

$$
\partial_{\phi}=\left(v_{i}^{\prime} \cdot w_{j}\right)=-\left(v_{i} \cdot w_{j}^{\prime}\right)=-\partial_{\phi^{\perp}}^{\text {transpose }} .
$$

The local ring at $x$ is a p.i.d., and any linear map between free modules over a p.i.d. can be diagonalized by changing the basis of the domain and codomain (cf. [J, p. 176]). Therefore, near $x$, we may assume $\partial_{\phi}$ is a diagonal matrix. In that case, in light of $(*)$, we see that the cokernels of $\partial_{\phi}$ and $\partial_{\phi^{\perp}}$ are isomorphic.

\section{EXAMPLES}

This section consists of several examples involving differential ranks and derived bundles. In (9.2), we describe all surjections of the form

$$
V_{\mathbb{P}^{1}} \rightarrow \mathscr{O}(1)^{\oplus r}
$$

where $\mathscr{O}(1)$ is the tautological bundle on projective space. We think of such a surjection as a join of lines in projective space and show how to decompose it into a join of osculating spaces to rational normal curves. In (9.3) and (9.4), we try to find relations among the differential ranks of a surjection $V_{X} \rightarrow E$, the dimension of $V$, and the rank of $E$. In (9.5), we classify the surjections with $\operatorname{deg} E=3$ using differential ranks and torsion sheaves. Finally, in (9.6), we show that every sequence of differential ranks and torsion numbers (subject to obvious restrictions) can occur on the projective line. 
Example 9.1. From the definition of differential ranks, it is immediate that

$$
\operatorname{drk}_{1} \phi \leq \min \left\{\mathrm{rk} S_{E}, \text { rk } E\right\} \text {. }
$$

Suppose that $\mathrm{rk} E=n-1$, and $E$ is generated by $n$ linearly independent global sections. Let $V \subset \Gamma(X, E)$ be the vector space spanned by these sections, and consider the natural map $\phi: V_{X} \rightarrow E$. It follows that the differential rank of $\phi$ is either one or zero. Therefore, either $E$ is trivial (at least in the case where the characteristic of $k$ is zero, (6.1.3)), or $E \cong \mathrm{G}^{m}(\mathscr{L}) \oplus W_{X}$ where $\mathscr{L}$ is a line bundle quotient $E$ and $W$ is a quotient of $V,(6.2 .2)$. In other words, $f: X \rightarrow G$ is the cone over an associated curve.

Example 9.2. Let $\mathscr{O}(1)$ be the tautological bundle on $\mathbb{P}_{k}^{1}$. We will determine the differential ranks of any surjection of the form

$$
\phi: V_{\mathbb{P}^{1}} \longrightarrow \mathscr{O}(1)^{\oplus r}
$$

where $V$ is some $k$-vector space of dimension $n$. (To avoid trivialities, we will always assume that $\phi$ is injective on global sections.) Geometrically, we are taking $r$ lines in $\mathbb{P}^{n-1}$ all isomorphic to some fixed $\mathbb{P}^{1}$, say $\psi_{i}: \mathbb{P}^{1} \stackrel{\sim}{\longrightarrow}$ $L_{i} \subset \mathbb{P}^{n-1}$ for $i=1, \ldots, r$, and considering the map to the Grassmannian of $(r-1)$-planes in $\mathbb{P}^{n-1}$

$$
\begin{aligned}
f: \mathbb{P}^{1} & \longrightarrow G_{r-1} \mathbb{P}^{n-1}=G(V, r), \\
p & \mapsto \operatorname{span}\left\{\psi_{1}(p), \ldots, \psi_{r}(p)\right\} .
\end{aligned}
$$

It is assumed that the $L_{i}$ and $\psi_{i}$ are chosen so that this map is defined everywhere. We will see that up to a change of coordinates on the Grassmannian, every such map is formed as follows: Take $n-r$ disjoint linear subspaces of $\mathbb{P}^{n-1}$

$$
\mathbb{P}^{a_{1}}, \mathbb{P}^{a_{2}}, \ldots, \mathbb{P}^{a_{n-r}}
$$

where $\sum a_{i}=r$. (The $a_{i}$ will be determined by the higher differential ranks.) At each point $p \in \mathbb{P}^{1}$, we can chose an osculating hyperplane to the rational normal curve of degree $a_{i}$ in $\mathbb{P}^{a_{i}}$ so that the span of these hyperplanes will be $f(p)$. Thus, we may say that $f$ comes from the join of the osculating developables of rational normal curves in disjoint linear subspaces of $\mathbb{P}^{n-1}$.

To describe this, let $W=\Gamma\left(\mathbb{P}^{1}, \mathscr{O}(1)\right)$. For each $m \geq 0$ consider the natural evaluation map

$$
S^{m} W \cong \Gamma\left(\mathbb{P}^{1}, \mathscr{O}(m)\right) \stackrel{\nu^{0}}{\longrightarrow} \mathscr{O}(m)
$$

corresponding to the rational normal curve of degree $m$ in $\mathbb{P}^{m}$, and consider the associated Taylor series map (A.6)

$$
S^{m} W \stackrel{\nu^{m-1}}{\longrightarrow} \mathrm{P}^{m-1}(\mathscr{O}(m))
$$

corresponding to the $(m-1)$-th associated map sending a point on the rational normal curve to its osculating hyperplane, (B.4, B.3.1).

On $\mathbb{P}^{1}$, every bundle is a direct sum of line bundles; so

$$
\operatorname{ker} \phi=\bigoplus_{i=1}^{n-r} \mathscr{O}\left(-a_{i}\right)
$$

where $\sum a_{i}=\operatorname{deg} \mathscr{O}(1)^{\oplus r}=r$. 
Theorem 9.2.2. Assume the characteristic of $k$ is zero or greater than $r$. There are isomorphisms giving a commutative diagram

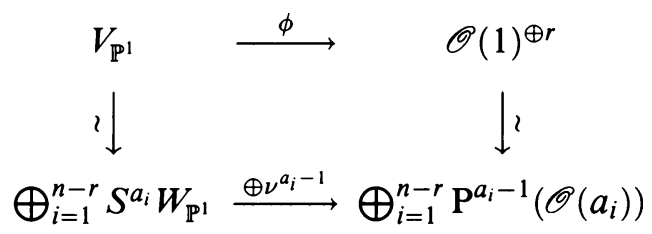

The torsion sheaves, $\operatorname{tor}_{i} \phi$, of $\phi$ are all zero, and the differential ranks are

$$
\operatorname{drk}_{i} \phi=\sharp\left\{j \mid a_{j} \geq i\right\} .
$$

In particular, $\mathrm{drk}_{1} \phi=n-r$.

Proof. From (A.3.2), it follows that the degree and rank of $\mathrm{P}^{m-1}(\mathscr{O}(m))$ are both $m$. Hence, there is an exact sequence

$$
0 \rightarrow \mathscr{O}(-m) \stackrel{j}{\longrightarrow} \Gamma\left(\mathbb{P}^{1}, \mathscr{O}(m)\right) \stackrel{\nu^{m-1}}{\longrightarrow} \mathrm{P}^{m-1}(\mathscr{O}(m)) \rightarrow 0 .
$$

For $m>0$, it is known, ([PS], [Pe2]), that

$$
\mathrm{P}^{m-1}(\mathscr{O}(m)) \cong \mathscr{O}(1)^{\oplus m} .
$$

Therefore the dual map, $j^{*}$, is injective on global sections. Counting dimensions implies that it is, in fact, an isomorphism on global sections. Therefore, we can choose an isomorphism between $\Gamma\left(\mathbb{P}^{1}, \mathscr{O}(m)\right)$ and its dual so that the following diagram commutes

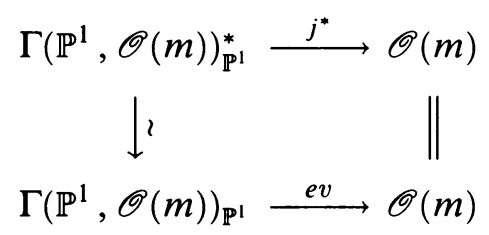

where $e v$ is the natural evaluation map.

Now consider the kernel, $K=\bigoplus_{i} \mathscr{O}\left(-a_{i}\right)$, of $\phi$ and the corresponding exact sequence

$$
0 \rightarrow K \rightarrow V_{\mathbb{P}^{1}} \stackrel{\phi}{\longrightarrow} \mathscr{O}(1)^{\oplus r} \longrightarrow 0 .
$$

Taking global sections shows that $\Gamma\left(\mathbb{P}^{1}, K\right)=0$ since we have assumed that $\phi$ is injective on global sections. Hence, $a_{i}>0$ for $i=1, \ldots, n-r$. Taking global sections of the dual of $(\dagger)$ shows that the natural map $V^{*} \rightarrow \Gamma\left(\mathbb{P}^{1}, K^{*}\right)$ is injective. Counting dimensions as before, shows that it is an isomorphism. Therefore, up to an automorphism of $V$, the map $V_{\mathbb{P}^{1}}^{*} \rightarrow K^{*}$ is

$$
\bigoplus_{i=1}^{n-r} \Gamma\left(\mathbb{P}^{1}, \mathscr{O}\left(a_{i}\right)\right) \stackrel{\oplus e v}{\longrightarrow} \bigoplus_{i=1}^{n-r} \mathscr{O}\left(a_{i}\right)=K^{*}
$$

Therefore, the required isomorphism, $(*)$, follows from $(* *)$.

To see the claim about the torsion divisors and differential ranks, first note that taking derived bundles and differential ranks commutes with direct sums. 
This follows, for example from (2.3) and the fact that the Taylor series maps respect direct sums, (A.6.3.1). The evaluation map

$$
\Gamma\left(\mathbb{P}^{1}, \mathscr{O}(m)\right)_{\mathbb{P}^{1}} \rightarrow \mathscr{O}(m)
$$

corresponds to a rational normal curve. It is well-known that such curves are noninflectionary, (B.3.1). This means that each associated Taylor series map

$$
\nu^{i}: \Gamma\left(\mathbb{P}^{1}, \mathscr{O}(m)\right)_{\mathbb{P}^{1}} \rightarrow \mathrm{P}^{i}(\mathscr{O}(m))
$$

is surjective (for $i \leq m$ ), i.e., $\mathrm{G}^{i}(\mathscr{O}(m))=\mathrm{P}^{i}(\mathscr{O}(m))$. Thus, it follows from Proposition 6.3.1, that the $j$-th derived bundle of $\mathrm{P}^{i}(\mathscr{O}(m))$ is $\mathrm{P}^{i-j}(\mathscr{O}(m))$. The claim about the higher differential ranks follows. The fact that the torsion sheaves of $\phi$ are zero follows by direct calculation (or by Theorem 8.1.1).

Remark 9.2.3. Further calculations in [Pe1] indicate that the differential ranks in the case just considered determine the generality of the positions of the lines, $L_{i}$.

Example 9.3. For $\phi: V_{\mathbb{P}^{1}} \rightarrow E$, if the dimension of $V$ is large enough relative to the degree of $E$, then the differential rank must be greater than one. Consider the line bundle $\mathscr{O}(d)$ on $\mathbb{P}^{1}$ with $d>0$. Let $V \subset \Gamma\left(\mathbb{P}^{1}, \mathscr{O}(d)\right)$ be a subvector space of dimension $n+1$ of globally generating sections, i.e., so that the corresponding map

$$
\phi: V_{\mathbb{P}^{1}} \rightarrow \mathscr{O}(d)
$$

is surjective. We have the osculating bundle of order $r,(\S 4$ and A.8):

$$
\mu^{r}: V_{\mathbb{P}^{1}} \rightarrow \mathrm{G}^{r}(\mathscr{O}(d)) .
$$

Proposition 9.3.1. Assume that the characteristic of $k$ is zero or greater than $d$; then

$$
\operatorname{deg} G^{r}(\mathscr{O}(d)) \geq(r+1)(n-r) .
$$

Proof. By Theorem B.2.3, rk $\mathrm{G}^{i}(\mathscr{O}(d))=i+1$ and

$$
\operatorname{deg} \mathrm{G}^{i}(\mathscr{O}(d))=(i+1)(d-i)-\sum_{j=1}^{i} \alpha_{j}
$$

for $i=1, \ldots, n$ where the $\alpha_{j}$ are the inflectionary numbers for $\phi$. In particular,

$$
\operatorname{deg} \mathrm{G}^{r}(\mathscr{O}(d))=(r+1)(d-r)-\sum_{i=1}^{r} \alpha_{i}
$$

and $V_{\mathbb{P}^{1}}=\mathrm{G}^{n}(\mathscr{O}(d))$ so that

$$
\sum_{i=1}^{n} \alpha_{i}=(n+1)(d-n) .
$$

Combining (*) and (**) and using the fact that $\alpha_{i} \leq \alpha_{i+1}$ gives

$$
\begin{aligned}
(* * *) \operatorname{deg} G^{r}(\mathscr{O}(d)) & =(r+1)(d-r)-(n+1)(d-n)+\alpha_{r+1}+\cdots+\alpha_{n} \\
& \geq(r+1)(d-r)-(n+1)(d-n)+(n-r) \alpha_{r} .
\end{aligned}
$$


Again using that $\alpha_{i} \leq \alpha_{i+1},(*)$ yields

$$
r \alpha_{r} \geq(r+1)(d-r)-\operatorname{deg} \mathrm{G}^{r}(\mathscr{O}(d)) .
$$

Along with $(* * *)$, this says

$$
\begin{aligned}
\operatorname{deg} G^{r}(\mathscr{O}(d)) & \geq(r+1)(d-r)-(n+1)(d-n) \\
& +\left(\frac{n-r}{r}\right)\left((r+1)(d-r)-\operatorname{deg} G^{r}(\mathscr{O}(d))\right) \\
\Longrightarrow \operatorname{deg} G^{r}(\mathscr{O}(d)) \geq & (r+1)(d-r)-\left(\frac{r}{n}\right)(n+1)(d-n) \\
\geq & (r+1)(d-r) \\
\geq & (r+1)(n-r) .
\end{aligned}
$$

Note. To see that equality is possible in Proposition 9.3.1, let $d=n$ and $V=$ $\Gamma\left(\mathbb{P}^{1}, \mathscr{O}(n)\right)$. Then $\mathrm{G}^{r}(\mathscr{O}(n))=\mathrm{P}^{r}(\mathscr{O}(n))$, (cf. B.3.1), and $\operatorname{deg} \mathrm{P}^{r}(\mathscr{O}(n))=$ $(r+1)(n-r)$, (cf. A.3.2).

Corollary 9.3.2. Assume the characteristic of $k$ is zero. Let $E$ be a vector bundle of rank $r$ on $\mathbb{P}^{1}$, and let $V \subset \Gamma\left(\mathbb{P}^{1}, E\right)$ be a subspace of dimension $n$ of generating sections. If the corresponding surjection

$$
V_{\mathbb{P}^{1}} \rightarrow E
$$

has differential rank one, then

$$
\operatorname{deg} E \geq r(n-r) .
$$

Proof. This follows directly from Corollary 6.2.2 and Proposition 9.3.1.

Example 9.4. Corollary 9.3.2 shows that if $n$ is large enough compared to the degree of $E$, then $\operatorname{drk}_{1} \phi>1$. It would be nice to find similar bounds for $n$ determining when $\mathrm{drk}_{1}$ is larger than any given value. At one extreme, if $V=\Gamma\left(\mathbb{P}^{1}, E\right)$ with $E$ any bundle (with no trivial factors) generated by global sections, then $\phi$ has full differential rank, i.e., $\operatorname{drk}_{1} \phi$ is as large as possible, namely $\mathrm{rk} E$, and $E_{1}=0$. The reason for this is that if $E=\bigoplus_{i=1}^{r} \mathscr{O}\left(n_{i}\right)$, then by (A.6.3.1), the Taylor series map $\nu_{\phi}^{1}$ breaks up into a direct sum of the Taylor series maps

$$
\nu^{1}: \Gamma\left(\mathbb{P}^{1}, \mathscr{O}\left(n_{i}\right)\right)_{\mathbb{P}^{1}} \rightarrow \mathrm{P}^{1}\left(\mathscr{O}\left(n_{i}\right)\right)
$$

for $i=1, \ldots, r$. Each of these Taylor series maps is surjective (by direct calculation or (B.3.1)). Thus, by (2.3), $E_{1}=0$.

We might expect that if $E$ is a bundle with no trivial factors on an arbitrary curve, $X$, and generated by global sections, then the natural map $\Gamma(X, E)_{X} \rightarrow$ $E$ would have full differential rank. But this is not the case:

Proposition 9.4.1. Let $E$ be an indecomposable bundle of rank $r$ on an elliptic curve $X$ with $2 r>\operatorname{deg} E>r$. Then $E$ is generated by global sections, but the natural evaluation map

$$
\phi: \Gamma(X, E)_{X} \rightarrow E
$$

does not have full differential rank, i.e., $E_{1} \neq 0$.

Proof. First note that such bundles, $E$, exist, $[\mathrm{Br}]$. If $\phi$ had full differential rank, then, by definition, the map

$$
\partial: S_{E} \rightarrow \Omega_{X / k} \otimes E
$$


is generically surjective. This says that

$$
\operatorname{dim} \Gamma(X, E) \geq 2 r
$$

(cf. 9.1). In our case, $\operatorname{deg} E>r$ implies that $E$ is generated by global sections and $\operatorname{dim} \Gamma(X, E)=\operatorname{deg} E<2 r,([\mathrm{Br}$, Lemma A1] $)$. Hence, $\phi$ cannot have full differential rank.

Example 9.5. We classify all surjections $V_{X} \rightarrow E$ where $X$ is an arbitrary smooth projective curve and $\operatorname{deg} E=3$. First, consider the bundle $E=\mathscr{O}(1) \oplus$ $\mathscr{O}(2)$ on $\mathbb{P}^{1}$. We want to see which differential ranks and torsion numbers are possible for a surjection

$$
\phi: V_{\mathbb{P}^{1}} \rightarrow E .
$$

We may assume by $(3.1)$ that $V \subset \Gamma\left(\mathbb{P}^{1}, E\right)$. To simplify matters, also assume that the characteristic of $k$ is zero.

$\operatorname{dim} V=5:$ If $\operatorname{dim} V=5$, then $V=\Gamma\left(\mathbb{P}^{1}, E\right)$, and $\phi$ is the ordinary evaluation map. It can be represented by the matrix

$$
\left[\begin{array}{ccccc}
x & y & 0 & 0 & 0 \\
0 & 0 & x^{2} & x y & y^{2}
\end{array}\right]
$$

Hence, the corresponding map $f: \mathbb{P}^{1} \rightarrow G_{1} \mathbb{P}^{4}$ comes from joining points on a line in $\mathbb{P}^{4}$ with matching points on a conic in a disjoint plane. The union of the lines $f(p)$ is a rational normal scroll. It is straightforward to check that $\operatorname{drk}_{1} \phi=2$, and that there is no torsion.

$\operatorname{dim} V=4$ : In this case, $\mathrm{drk}_{1}=2$ and tor ${ }_{1} \phi$ has length two. If the differential rank were one, then $E_{1}$ would be a line bundle, and comparing ranks, the natural map $E \rightarrow \mathrm{G}^{1}\left(E_{1}\right)$ of (4.2) would be an isomorphism. Proposition 9.3.1 shows this is not possible. Thus, $\mathrm{drk}_{1}=2$ and

$$
\partial: S_{E} \rightarrow \Omega_{X / k} \otimes E
$$

is injective and generically surjective. The first torsion number is

$$
\text { length }(\operatorname{cok} \partial)=\operatorname{deg}\left(\Omega_{X / k} \otimes E\right)-\operatorname{deg} S_{E}=2 .
$$

The torsion sheaf can be supported at one point, e.g.,

$$
\phi=\left[\begin{array}{cccc}
x & y & 0 & 0 \\
0 & x^{2} & x y & y^{2}
\end{array}\right]
$$

or it can be supported at two points, e.g.,

$$
\phi=\left[\begin{array}{cccc}
x & 0 & y & 0 \\
0 & x^{2} & x y & y^{2}
\end{array}\right] .
$$

Direct calculation shows that in $(*)$, tor $_{1} \phi$ is supported at the point $y=0$ and in $(* *)$ it is supported at the points $x=0$ and $y=0$.

Consider the maps $f, g: \mathbb{P}^{1} \rightarrow G_{1} \mathbb{P}^{3}$ corresponding to $(*)$, (**), respectively. The associated loci

$$
\{f(p)\}_{p \in \mathbb{P}^{1}}, \quad\{g(p)\}_{p \in \mathbb{P}^{1}}
$$

are the two types of cubic ruled surfaces in $\mathbb{P}^{3}$, (cf. [E, $\left.\left.\S 37\right]\right)$. The former comes from projecting the rational normal scroll of degree three in $\mathbb{P}^{4}$ that we just 
considered from a point lying on a tangent to the scroll. The latter comes from projecting from a general point.

$\operatorname{dim} V=3$ : If $\operatorname{dim} V=3$, then $S_{E}=\mathscr{O}(-3)$ is a line bundle; so $\operatorname{drk}_{1} \phi=1$. By the Piene duality theorem, (7.2.1), there is a commutative diagram

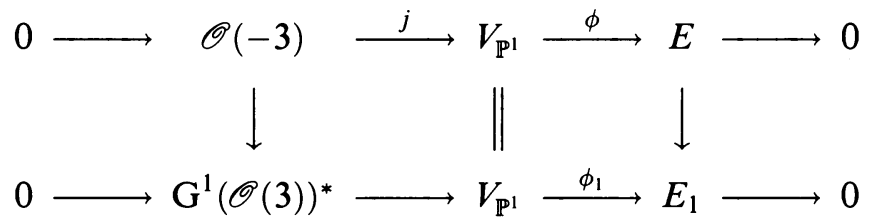

The map $\mathbb{P}^{1} \rightarrow \mathbb{P}^{2}$ corresponding to $\phi_{1}$ is the dual of that corresponding to $j^{*}: V^{*} \rightarrow \mathscr{O}(3)=S_{E}^{*}$. So there are two possibilities, depending on whether $j^{*}$ represents a nodal cubic or a cuspidal cubic. If the former, then $E_{1} \cong \mathscr{O}(4)$ since the dual of the nodal cubic is a quartic. From the exact sequences

$$
0 \rightarrow S_{E} \stackrel{\partial}{\longrightarrow} \Omega_{X / k} \otimes E \rightarrow \operatorname{cok} \partial \rightarrow 0
$$

and

$$
0 \longrightarrow \operatorname{tor}_{1} \phi \rightarrow \operatorname{cok} \partial \longrightarrow \Omega_{X / k} \otimes E_{1} \rightarrow 0
$$

it follows that $\operatorname{tor}_{1} \phi=0$.

If $j^{*}$ corresponds to a cuspidal cubic, then $E_{1} \cong \mathscr{O}(3)$ since the dual of a cuspidal cubic is a cuspidal cubic. The exact sequences $(\dagger),(\ddagger)$ show that length $\left(\operatorname{tor}_{1} \phi\right)=1$.

9.5.1. Arbitrary curves of degree three. Let $E$ be a vector bundle of rank $r$ and degree three on an arbitrary curve, $X$, and let $V \subset \Gamma(X, E)$ be a subspace of generating sections with evaluation map

$$
\phi: V_{X} \rightarrow E \text {. }
$$

To avoid trivial cases, assume that the corresponding map

$$
f: X \rightarrow G(V, r) \stackrel{\text { Plücker }}{\longrightarrow} \mathbb{P}^{N}
$$

expresses $X$ as a cubic curve in $\mathbb{P}^{N}$, i.e., $f$ is birational to its image. Therefore, $X$ is either a twisted cubic or a plane cubic. So either $X$ is rational, and we are reduced to the case just considered in example (9.5), or $X$ is elliptic. If $X$ is elliptic, write $E=\bigoplus_{i=1}^{m} E^{i}$ where each $E^{i}$ is indecomposable. Since each $E^{i}$ is generated by global sections, either $E^{i} \cong \mathscr{O}_{X}$ or $\operatorname{deg} E^{i}>\mathrm{rk} E^{i}$, ([Br, Lemma A1]). Therefore, forgetting about trivial factors, (6.1), there are two possibilities:

(a) $E$ is a line bundle and $\phi$ corresponds to a smooth plane cubic.

(b) $E$ is an indecomposable bundle of rank two. Then $\operatorname{dim} \Gamma(X, E)=$ 3 , $([\mathrm{Br}, \mathrm{A} .4])$; so $V=\Gamma(X, E)$. By the duality theorem, (7.2.1), there is a commutative diagram

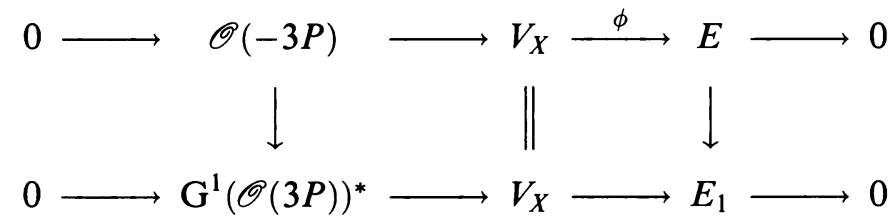


for some point $P \in X$. By (B.3.4) and Theorem B.2.3, $\mathrm{G}^{1}(\mathscr{O}(3 P))=\mathrm{P}^{1}(\mathscr{O}(3 P))$ so $\operatorname{deg} E_{1}=\operatorname{deg} G^{1}(\mathscr{O}(3 P))=6$. The map $f: X \rightarrow G_{1} \mathbb{P}^{3}$ from $\phi$ gives the envelope of tangent lines to a plane elliptic sextic. From the sequences $(\dagger)$ and $(\ddagger)$, it follows that $\operatorname{tor}_{1} \phi=0$.

Example 9.6. If the characteristic of $k$ is zero or greater than $t$, then any sequence of differential ranks

$$
d_{1} \geq \cdots \geq d_{t} \geq d_{t+1}=0
$$

can occur. For example, on $\mathbb{P}^{1}$, consider the bundle

$$
E=\bigoplus_{i=0}^{t-1} \mathrm{P}^{i}(\mathscr{O}(i+1))^{\oplus\left(d_{i+1}-d_{i+2}\right)}
$$

For each $i$, there is the natural Taylor series map

$$
\nu^{i}: \Gamma\left(\mathbb{P}^{1}, \mathscr{O}(i+1)\right)_{\mathbb{P}^{1}} \rightarrow \mathrm{P}^{i}(\mathscr{O}(i+1)) .
$$

Take a direct sum of $d_{i+1}-d_{i+2}$ copies of this map and then sum over $i$ to get

$$
\phi: V_{\mathbb{P}^{1}}=\left[\bigoplus_{i=0}^{t-1} \Gamma\left(\mathbb{P}^{1}, \mathscr{O}(i+1)\right)^{\oplus\left(d_{i+1}-d_{i+2}\right)}\right]_{\mathbb{P}^{1}} \rightarrow E .
$$

By (A.6.3.1) and Proposition 6.3.1

$$
E_{j}=\bigoplus_{i=j}^{t-1} \mathrm{P}^{i-j}(\mathscr{O}(i+1))^{\oplus\left(d_{i+1}-d_{i+2}\right)} .
$$

Therefore,

$$
\begin{aligned}
\operatorname{drk}_{j} \phi & =\mathrm{rk} E_{j-1}-\mathrm{rk} E_{j} \\
& =\sum_{i=j-1}^{t-1}(i-j+2)\left(d_{i+1}-d_{i+2}\right)-\sum_{i=j}^{t-1}(i-j+1)\left(d_{i+1}-d_{i+2}\right) \\
& =\sum_{i=j-1}^{t-1}\left(d_{i+1}-d_{i+2}\right) \\
& =d_{j} .
\end{aligned}
$$

Example 9.7. Any sequence of nonnegative integers

$$
k_{1}, \ldots, k_{n}
$$

can occur as the sequence of torsion numbers at a point. To see this, let

$$
\alpha_{i}=\sum_{j=1}^{i} k_{n-j+1}
$$

for $i=1, \ldots, n$, and define the map

$$
\begin{aligned}
f: \mathbb{C} & \rightarrow G\left(\mathbb{C}^{\oplus n+1}\right) \cong \mathbb{P}^{n} \\
z & \mapsto\left(1, z^{1+\alpha_{1}}, z^{2+\alpha_{2}}, \ldots, z^{t+\alpha_{n}}\right) .
\end{aligned}
$$


Let $\mathscr{L}$ be the line bundle, $\mathbb{C} \times \mathbb{C}$, on $\mathbb{C}$, and consider the map of $\S 4$,

$$
\mu^{n-1}: \mathbb{C}^{n+1} \rightarrow \mathrm{G}^{n-1}(\mathscr{L}) .
$$

It suffices to check the following

Claim. The $i$-th torsion number of $\mu^{n-1}$ is $\alpha_{n-i+1}-\alpha_{n-i}=k_{i}$.

Sketch of proof. We need a local version of Theorem 8.1.1. The map $\delta$ of (A.7) induces a commutative diagram with exact rows

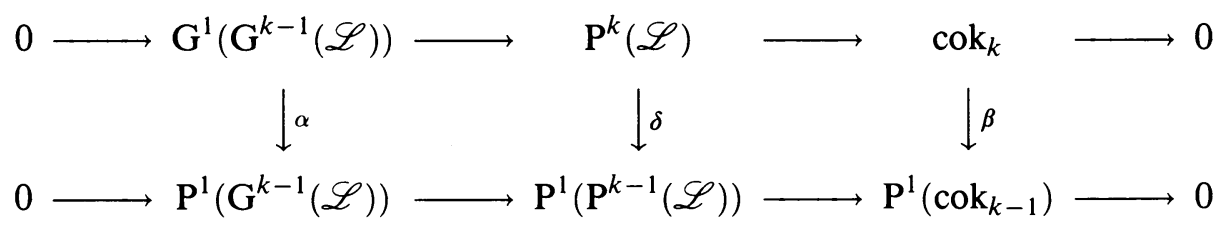

The map $\alpha$ comes from the Taylor series map $\mathbb{C}^{n+1} \rightarrow \mathrm{P}^{1}\left(\mathrm{G}^{k-1}(\mathscr{L})\right)$. By Proposition 6.3.1, the $i$-th derived bundle of $\mu^{n-1}$ is $\mathrm{G}^{n-i-1}(\mathscr{L})$. Hence, cok $\alpha$ modulo torsion is $\Omega_{X / k} \otimes \mathrm{G}^{k-2}(\mathscr{L})$, (cf. 2.3).

By (A.8.3), $\mathrm{G}^{1}\left(\mathrm{G}^{k-1}(\mathscr{L})\right) \cong \mathrm{G}^{k}(\mathscr{L})$. Therefore, in the diagram, $\operatorname{cok}_{i}$ is the cokernel of the $i$-th Taylor series map, $i=k-1, k$. Theorem B.2.3, (cf. B.2.3.3) yields

$$
\text { length }\left(\operatorname{cok}_{k}\right)=\alpha_{1}+\cdots+\alpha_{k} \text {. }
$$

Similarly, length $\left(\operatorname{cok}_{k-1}\right)=\alpha_{1}+\cdots+\alpha_{k-1}$; hence, the fundamental exact sequence, (A.3.2), shows that

$$
\text { length }{ }_{z}\left(\mathbf{P}^{1}\left(\operatorname{cok}_{k-1}\right)\right)=2\left(\alpha_{1}+\cdots+\alpha_{k-1}\right) \text {. }
$$

By Proposition A.7.8, $\delta$ is an inclusion. Thus, the snake lemma gives an exact sequence

$$
0 \rightarrow \operatorname{cok} \alpha / \operatorname{ker} \beta \rightarrow \operatorname{cok} \delta \rightarrow \operatorname{cok} \beta \rightarrow 0 .
$$

The proof of Proposition A.7.8 shows that $\operatorname{cok} \delta=\Omega_{X / k} \otimes \mathrm{P}^{k-2}(\mathscr{L})$. Hence, $\operatorname{cok} \alpha / \operatorname{ker} \beta$ is torsion free; it is the $(n-k+1)$-th derived bundle of $\mu^{n-1}$, $\Omega_{X / k} \otimes \mathrm{G}^{k-2}(\mathscr{L})$, and $\operatorname{ker} \beta$ is the corresponding torsion divisor. The map $\operatorname{cok} \alpha / \operatorname{ker} \beta \hookrightarrow \operatorname{cok} \delta$ is just the natural inclusion, $\mathrm{G}^{k-2}(\mathscr{L}) \rightarrow \mathrm{P}^{k-2}(\mathscr{L})$ tensored by $\Omega_{X / k}$. Hence,

$$
\text { length }{ }_{z}(\operatorname{cok} \beta)=\alpha_{1}+\cdots+\alpha_{k-2} \text {. }
$$

It follows from $(*)$ and $(* *)$ that the $(n-k+1)$-th torsion number is

$$
\text { length }{ }_{z}(\operatorname{ker} \beta)=\alpha_{k}-\alpha_{k-1}
$$

as required.

\section{APPENDIX A}

Introduction. This appendix gathers together the main facts about principal parts sheaves and is intended as a technical reference for the paper. For the most part, it is a compilation of results found in [G], [K1], and [Pi1], worked out in the detail necessary for our applications. Propositions 7.8 and 8.3 , used throughout the paper, are new results. 
A.1. Definition of the principal parts sheaf. Let $u: X \rightarrow S$ be a morphism of schemes and $\mathscr{F}$ an $\mathscr{O}_{X}$-module. Let $\Delta_{(t)}$ be the $t$-th infinitesimal neighborhood of the diagonal. It is defined as a subscheme of $X \times_{S} X$ by the ideal $\mathscr{J}^{t+1}$ where $\mathscr{F}$ is the ideal of the ordinary diagonal $\Delta=\Delta_{(0)}$. In the following diagram, let $i$ denote the inclusion map and $\pi_{1}, \pi_{2}$ the natural projections:

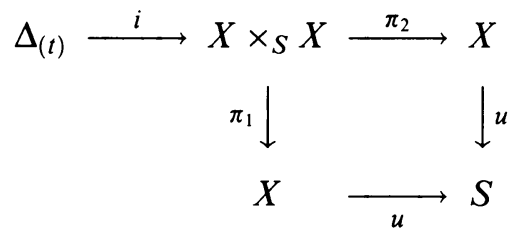

Let $p=\pi_{1} \circ i$ and $q=\pi_{2} \circ i$.

Definition 1.2. $\quad \mathrm{P}^{t}(\mathscr{F})=p_{*} q^{*} \mathscr{F}=\pi_{1 *}\left(\mathscr{O}_{X \times X} / \mathscr{I}^{t+1} \otimes \pi_{2}^{*} \mathscr{F}\right)$ is called the sheaf of $t$-th order principal parts of $\mathscr{F}$ over $S$ or the $t$-jets of sections of $\mathscr{F}$ over $S$.

Applying $\pi_{1 *}$ the the natural surjection

$$
\mathscr{O}_{X \times X} / \mathscr{I}^{t+1} \otimes \pi_{2}^{*} \mathscr{F} \rightarrow \mathscr{O}_{X \times X} / \mathscr{I}^{t} \otimes \pi_{2}^{*} \mathscr{F}
$$

gives a map

$$
\mathrm{P}^{t}(\mathscr{F}) \rightarrow \mathrm{P}^{t-1}(\mathscr{F})
$$

which is a surjection since the sheaves on $(*)$ are supported on $\Delta$ and $\left.\pi_{1}\right|_{\Delta}$ is a homeomorphism. Since tensor products respect direct sums, so does this surjection.

A.2. Functorial properties. $\mathscr{F} \rightarrow \mathbf{P}^{t}(\mathscr{F})$ is a covariant functor from the category of $\mathscr{O}_{X}$-modules to itself. In this section we summarize its most basic properties.

2.1. If $X$ is noetherian, $u$ of finite type, and $\mathscr{F}$ quasi-coherent, then $\mathrm{P}^{t}(\mathscr{F})$ is quasi-coherent. If $X$ is noetherian, $X \rightarrow S$ proper, and $\mathscr{F}$ coherent, then $\mathrm{P}^{t}(\mathscr{F})$ is coherent, ([H, II.5.8]). For $X \rightarrow S$ smooth, if $\mathscr{F}$ is locally free, so is $\mathrm{P}^{t}(\mathscr{F}),($ Proposition 3.3).

2.2. Since $q^{*}$ and $p_{*}$ preserve direct sums, so does $\mathrm{P}^{t}(\cdot)$.

2.3. If we restrict $\mathrm{P}^{t}(\cdot)$ to act on locally free sheaves, then it is exact. This is because pulling back locally free sheaves is always exact and $p_{*}$ is exact since it is a homeomorphism.

2.4. Let $f: X \rightarrow Y$ be a morphism of schemes over $S$. There is a map

$$
f^{*} \mathrm{P}^{t}(\mathscr{F}) \rightarrow \mathrm{P}^{t}\left(f^{*} \mathscr{F}\right) .
$$

This map is mentioned in [K1] and in [Pil]; we construct it in the proof of Proposition 3.4, below. In particular, if $X \rightarrow S$ is a finite type, separated morphism of noetherian schemes, e.g., a variety over a field, and $U \subset X$ is an open subscheme, then $\left.\mathrm{P}^{t}(\mathscr{F})\right|_{U} \cong \mathrm{P}^{t}\left(\left.\mathscr{F}\right|_{U}\right)$ : this follows from a commutativity property of pullbacks and pushforwards, ([H, II.9.3]). We will only need this result in the special case where $X \rightarrow S$ is smooth and $\mathscr{F}$ is locally free, (Corollary 3.5). 
2.5. It is clear from the definition of the natural surjection $\mathrm{P}^{t}(\mathscr{F}) \rightarrow \mathrm{P}^{t-1}(\mathscr{F})$ of (1.3), that it is functorial, i.e., given a map of sheaves $\mathscr{F} \rightarrow \mathscr{G}$, there is a natural commutative diagram

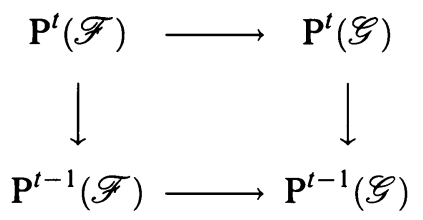

2.6. It is also clear that the natural surjections of (1.3) preserve direct sums.

A.3. Fundamental exact sequence. Assume now that $\mathscr{I}$ is locally generated by a regular sequence, e.g., $X \rightarrow S$ smooth, and assume $\mathscr{F}$ is locally free of rank r. Apply $\pi_{1 *}\left(\cdot \otimes \pi_{2}^{*} \mathscr{F}\right)$ to the exact sequence

$$
0 \rightarrow \mathscr{I}^{t} / \mathscr{I}^{t+1} \rightarrow \mathscr{O}_{X \times X} / \mathscr{I}^{t+1} \rightarrow \mathscr{O}_{X \times X} / \mathscr{I}^{t} \rightarrow 0
$$

to get the fundamental exact sequence

$$
0 \rightarrow S^{t}\left(\Omega_{X / S}\right) \otimes \mathscr{F} \rightarrow \mathrm{P}^{t}(\mathscr{F}) \rightarrow \mathrm{P}^{t-1}(\mathscr{F}) \rightarrow 0
$$

where $S^{t}\left(\Omega_{X / S}\right)$ denotes the $t$-th symmetric power of the relative cotangent bundle. One assumes that $\mathscr{I}$ is locally generated by a regular sequence so that $\mathscr{I}^{t} / \mathscr{I}^{t+1} \cong S^{t}(\Omega),[\mathrm{F}, \mathrm{A} .6 .1]$. Note that $\pi_{1 *}$ preserves the exactness of (3.1) since the sheaves involved are supported on $\Delta$ which is homeomorphic to $X$. By induction and the fact that $\mathrm{P}^{0}(\mathscr{F})=\mathscr{F}$ we get

Proposition 3.3. Suppose $X$ has dimension $n$. Let $X \rightarrow S$ be smooth and $\mathscr{F}$ a locally free sheaf of rank $r$ on $X$. Then $\mathrm{P}^{t}(\mathscr{F})$ is locally free of rank $r \cdot\left(\begin{array}{c}n+t \\ t\end{array}\right)$.

Given a map as in (2.4), there is a corresponding map between fundamental exact sequences:

Proposition 3.4. Let $X$ and $Y$ be smooth $S$-schemes and $\mathscr{F}$ a locally free sheaf on $Y$. If $f: X \rightarrow Y$ is a morphism of $S$-schemes, the map of (2.4) induces a map of exact sequences

$$
0 \longrightarrow S^{t}\left(f^{*} \Omega_{Y / S}\right) \otimes f^{*} \mathscr{F} \longrightarrow f^{*} \mathrm{P}^{t}(\mathscr{F}) \longrightarrow f^{*} \mathrm{P}^{t-1}(\mathscr{F}) \longrightarrow 0
$$

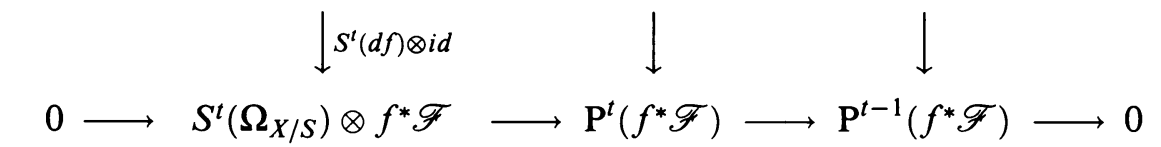

where $d f: f^{*} \Omega_{Y / S} \rightarrow \Omega_{X / S}$ is the cotangent map.

Proof. Using the notation in (A.1), let $\pi_{X, i}=\pi_{i}$ for $i=1,2$. There is corresponding diagram for defining principal parts bundles on $Y$ with projections $\pi_{Y, i}, i=1,2$. Let $\mathscr{I}$ (respectively, $\mathscr{J}$ ) be the ideal of the diagonal in $X \times_{S} X$ (respectively, $Y \times_{S} Y$ ). The map $f$ induces a commutative diagram

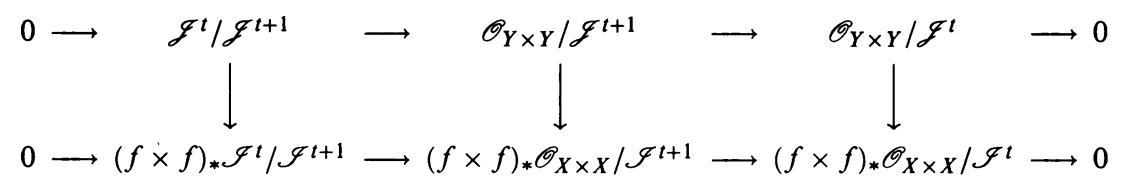


Apply $f^{*} \pi_{Y, 1 *}\left(\cdot \otimes \pi_{Y, 2}^{*} \mathscr{F}\right)$ to this diagram to get

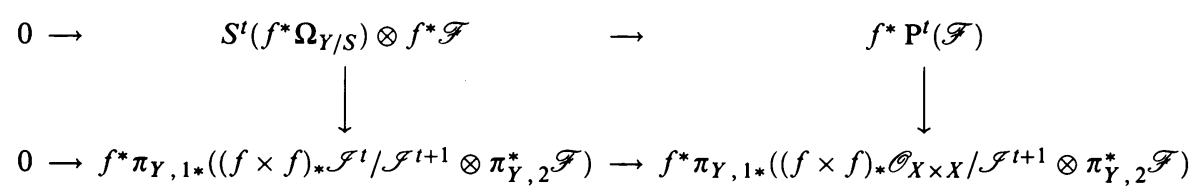

(continuing from above)

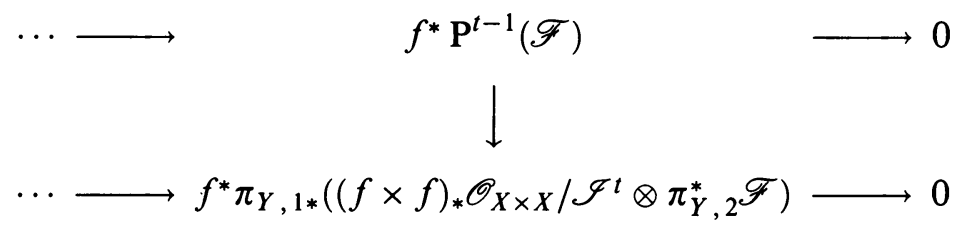

Again, we are using the smoothness of $Y \rightarrow S$ to identify $\mathcal{J}^{t} / \mathcal{J}^{t+1}$ with $S^{t}\left(\Omega_{Y / S}\right)$. We also use the standard fact that taking symmetric powers commutes with pullbacks.

Now use the maps

$$
\begin{aligned}
f^{*} \pi_{Y, 1 *} & \rightarrow \pi_{X, 1 *}(f \times f)^{*}, \\
(f \times f)^{*} \pi_{Y, 2}^{*} & =\pi_{X, 2}^{*} f^{*}, \\
\pi_{Y, 1 *}(f \times f)_{*} & =f_{*} \pi_{X, 1 *}, \\
f^{*} f_{*} & \rightarrow 1 .
\end{aligned}
$$

(For the first map, cf. [H, II.9.3].) Applying these to the first sheaf on the bottom of the previous diagram yields

$$
\begin{aligned}
f^{*} \pi_{Y, 1 *}\left((f \times f)_{*} \mathscr{I}^{t} / \mathscr{I}^{t+1} \otimes \pi_{Y, 2}^{*} \mathscr{F}\right) \\
=f^{*} \pi_{Y, 1 *}(f \times f)_{*} \mathscr{I}^{t} / \mathscr{I}^{t+1} \otimes f^{*} \pi_{Y, 1 *} \pi_{Y, 2}^{*} \mathscr{F} \\
=f^{*} f_{*} \pi_{X, 1 *} \mathscr{I}^{t} / \mathscr{I}^{t+1} \otimes \pi_{X, 1 *}(f \times f)^{*} \pi_{Y, 2}^{*} \mathscr{F} \\
\quad \rightarrow \pi_{X, 1 *} \mathscr{J}^{t} / \mathscr{I}^{t+1} \otimes \pi_{X, 1 *} \pi_{X, 2}^{*} f^{*} \mathscr{F} \\
=S^{t} \Omega_{X / S} \otimes f^{*} \mathscr{F} .
\end{aligned}
$$

Similarly, $f^{*} \pi_{Y, 1 *}\left((f \times f)_{*} \mathscr{O}_{X \times X} / \mathscr{J}^{t+1} \otimes \pi_{Y, 2}^{*} \mathscr{F}\right)=\mathrm{P}^{t}(\mathscr{F})$. Finally, a similar argument applies to the sheaf on the bottom right of the diagram. We thus get $(*)$. The leftmost vertical map of $(*)$ has the right form since $d f$ comes from the natural map

$$
\mathscr{J} / \mathscr{J}^{2} \rightarrow(f \times f)_{*} \mathscr{I} / \mathscr{J}^{2}
$$

while the leftmost vertical map of the first diagram of this proof is obtained by taking symmetric powers of this map

$$
\mathscr{J}^{t} / \mathscr{J}^{t+1}=S^{t}\left(\mathscr{J} / \mathscr{J}^{2}\right) \rightarrow(f \times f)_{*} S^{t}\left(\mathscr{I} / \mathscr{I}^{2}\right)=(f \times f)_{*} \mathscr{I}^{t} / \mathscr{I}^{t+1}
$$

(cf. [F, B.7.1]).

Corollary 3.5. Let $U$ be an open subset of a smooth $S$-scheme $X$, and let $\mathscr{F}$ be a locally free sheaf on $X$. Then

$$
\left.\mathrm{P}^{t}(\mathscr{F})\right|_{U}=\mathrm{P}^{t}\left(\left.\mathscr{F}\right|_{U}\right)
$$

Proof. Apply Proposition 3.4 to the inclusion $f: U \hookrightarrow X$. The case $t=1$ follows from the 5-lemma and the fact that $\Omega_{U / S}=\left.\Omega_{X / S}\right|_{U}$. The general case 
follows from Proposition 3.4 by induction using the 5-lemma and the fact that pullbacks commute with taking symmetric powers:

$$
S^{t}\left(\Omega_{U / S}\right)=S^{t}\left(f^{*} \Omega_{X / S}\right)=f^{*} S^{t}\left(\Omega_{X / S}\right) .
$$

Note. As mentioned earlier, Corollary 3.5 is true more generally for $X \rightarrow S$ a finite type, separated morphism of Noetherian schemes and $\mathscr{F}$ any sheaf. However, we will not need this in the paper.

A.4. Local trivialization of $\mathrm{P}^{t}(E)$. Let $X \rightarrow S$ be smooth and $E$ be a bundle (locally free sheaf) on $X$. This section gives local coordinates for the jets of sections of $E$. To make notation simpler, we will mainly consider the case where $X \rightarrow S$ has relative dimension 1, e.g., a nonsingular curve over an algebraically closed field. Remark 4.9 , at the end of this section, discusses the modifications necessary for higher relative dimensions.

Let $U=\operatorname{Spec} A$ be an open affine subset of $X$ and $V=\operatorname{Spec} B$ be an open affine subset of $S$ with $U \subset u^{-1}(V)$. Let $I$ denote the kernel of the map

$$
\begin{aligned}
A \otimes_{B} A & \rightarrow A \\
a \otimes b & \mapsto a b .
\end{aligned}
$$

So $\left.\tilde{I} \cong \mathscr{I}\right|_{U}$, and $\left.\widetilde{I / I^{2}} \cong \Omega\right|_{U}$, the relative cotangent bundle of $X \rightarrow S$ restricted to $U$. The ideal $I$ is generated by $\{d a\}_{a \in A}$ where $d a=1 \otimes a-a \otimes 1$, [M, Chapter 9]. With this notation, the exact sequence (3.2) restricted to $U$ and with $\mathscr{F}=\mathscr{O}_{X}$ becomes

$$
0 \rightarrow I^{t} / I^{t+1} \longrightarrow\left(A \otimes_{B} A\right) / I^{t+1} \rightarrow\left(A \otimes_{B} A\right) / I^{t} \rightarrow 0
$$

We are free to identify $\left.\Omega^{\otimes t}\right|_{U}$ with $I^{t} \widetilde{I^{t+1}}$ since $X \rightarrow S$ is smooth. (We have also used that $\left.\mathrm{P}^{t}(E)\right|_{U} \cong \mathrm{P}^{t}\left(\left.E\right|_{U}\right),(2.4)$.)

Now take $U$ small enough so that $\left.\Omega\right|_{U}$ is trivial with generator $d z=1 \otimes z-$ $z \otimes 1$. By induction on $t$, it follows that $\left(A \otimes_{B} A\right) / I^{t+1}$ is a free $A$-module with basis the image of $\left\{(d z)^{i}\right\}_{i=0}^{t}$. Define a map $\partial_{z}: A \rightarrow A$ by the formula $d b=1 \otimes b-b \otimes 1=\partial_{z} b d z$. Inductively, define

$$
\partial_{z}^{i}: A \rightarrow A
$$

by $\partial_{z}^{i} b=\partial_{z}\left(\partial_{z}^{i-1} b\right)$. Finally, define the map

$$
\begin{aligned}
\beta_{t}:(A \otimes A) / I^{t+1} & \rightarrow A^{\oplus t+1} \\
a \otimes b & \mapsto a\left(b, \partial_{z} b, \frac{1}{2 !} \partial_{z}^{2} b, \ldots, \frac{1}{t !} \partial_{z}^{t} b\right) .
\end{aligned}
$$

Note 4.3. In order for this map to make sense, we make the assumption that the characteristic of each residue field of $S$ is zero or greater than $t$.

Note 4.4. If $X$ is a nonsingular curve over an algebraically closed field $k=$ $S$, the completion of the local ring $\mathscr{O}_{X, x}$ at a point $x \in X$ is the ring of formal power series $k[[z]]$ in a local parameter $z$. By including $\mathscr{O}_{X, x}$ in its completion, we may interpret $\partial_{z}^{i} b$ as formal differentiation with respect to $z$.

We will see that $\beta_{t}$ is well-defined as part of 
Proposition 4.5. $\quad \beta_{t}$ is an isomorphism.

Proof. It suffices to show that $\beta_{t}\left(d z^{j}\right)=(0, \ldots, 0,1,0, \ldots, 0)$ with the 1 in the $(j+1)$-th component. Since $d z^{t+1} \mapsto 0, \beta_{t}$ is well-defined. The $(\ell+1)$-th component of $\beta_{t}\left(d z^{j}\right)$ is

$$
\begin{aligned}
\beta_{t}\left(d z^{j}\right)_{\ell+1} & =\beta_{t}\left((1 \otimes z-z \otimes 1)^{j}\right)_{\ell+1} \\
& =\sum_{i=0}^{j}(-1)^{i}\left(\begin{array}{c}
j \\
i
\end{array}\right) \frac{1}{\ell !} z^{i} \partial_{z}^{\ell} z^{j-i} \\
& =z^{j-\ell} \sum_{i=1}^{j}(-1)^{i}\left(\begin{array}{c}
j \\
i
\end{array}\right)\left(\begin{array}{c}
j-i \\
\ell
\end{array}\right)
\end{aligned}
$$

where $\left(\begin{array}{l}a \\ b\end{array}\right)=0$ for $b>a$. However,

$$
\sum_{i=1}^{j}(-1)^{i}\left(\begin{array}{c}
j \\
i
\end{array}\right)\left(\begin{array}{c}
j-i \\
\ell
\end{array}\right)=\left.\frac{1}{\ell !} \partial_{z}^{\ell}(z-1)^{j}\right|_{z=1}= \begin{cases}1, & j=\ell, \\
0, & j \neq \ell,\end{cases}
$$

as required.

Let $E$ be an arbitrary vector bundle of rank $r$. Using (2.2) and the fact that $\left.\mathrm{P}^{t}(E)\right|_{U} \cong \mathrm{P}^{t}\left(\left.E\right|_{U}\right)$, Proposition 4.5 allows us to construct a local trivialization of $\mathrm{P}^{t}(E)$. Use the notation from above and assume, in addition, that a trivialization $\left.E\right|_{U} \cong \mathscr{O}_{U}^{\oplus r}$ is chosen. Then

$$
\Gamma\left(U, \mathrm{P}^{t}(E)\right) \cong \frac{A \otimes A}{I^{t+1}} \otimes_{A} A^{\oplus r} \cong\left(\frac{A \otimes A}{I^{t+1}}\right)^{\oplus r} \stackrel{\beta_{t}^{\oplus r}}{\longrightarrow}\left(A^{\oplus t+1}\right)^{\oplus r}
$$

where we consider $(A \otimes A) / I^{t+1}$ as a right $A$-module for the purpose of tensoring over $A$. By abuse of notation, we will call the composite of the above maps

$$
\beta_{t}: \Gamma\left(U, \mathrm{P}^{t}(E)\right) \longrightarrow\left(A^{\oplus t+1}\right)^{\oplus r}
$$

Restricting (3.2) to $U$ gives an isomorphism of exact sequences (4.7)

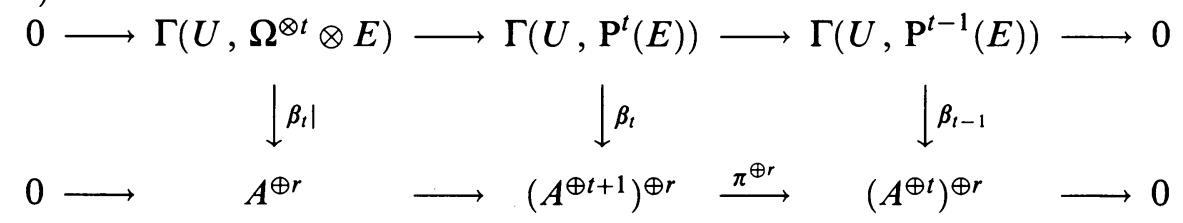

The inclusion of $A^{\oplus t}$ in $A^{\oplus t+1}$ as the first $t$ factors induces a map $\left(A^{\oplus t}\right)^{\oplus r} \rightarrow$ $\left(A^{\oplus t+1}\right)^{\oplus r}$ giving a splitting of the rows of (4.7). In sum, we have

Corollary 4.8. $A$ choice of a local trivialization of $\Omega_{X / S}$ and $E$ determines a local trivialization of $\mathrm{P}^{t}(E)$ for each $t$ and local splittings of the fundamental exact sequence (3.2).

Remark 4.9. The analysis given in this section requires little change if the dimension of $\mathrm{X}$ is $n>1$, the main difference being that in defining the map $\beta_{t}$ of (4.2), one must consider all mixed partials with respect to the local coordinates on $X$. To be specific, consider the case of $\mathrm{P}^{t}(\mathscr{L})$ where $\mathscr{L}$ is a line bundle. Take an open affine $U=\operatorname{Spec} A$ so that $\left.\mathscr{L}\right|_{U} \cong \mathscr{O}_{U}$ and $\left.\Omega\right|_{U} \cong \widetilde{I / I^{2}}$ as before. 
Take $U$ small enough so that $\left.\Omega\right|_{U}$ is trivial, say $\left.\mathscr{O}_{U}^{\oplus r} \cong \Omega\right|_{U}$. Take the images of the standard basis elements of $\mathscr{O}_{U}^{\oplus n}$ to get generators $d z_{i}=1 \otimes z_{i}-d z_{i} \otimes 1$, $i=1, \ldots, n$, for $\left.\Omega\right|_{U}$. Define a map

$$
\begin{aligned}
& \beta_{t}:(A \otimes A) / I^{t+1} \rightarrow A^{\oplus\left(\begin{array}{c}
n+t \\
t
\end{array}\right)} \\
& a \otimes b \mapsto a\left\{\frac{1}{i_{1} ! \cdots i_{j} !} \partial_{i_{1}} \cdots \partial_{i_{j}} b\right\}_{0 \leq j \leq t,\left\{i_{1}, \ldots, i_{j}\right\} \subset\{1, \ldots, n\}}
\end{aligned}
$$

where $\partial_{i} b$ is defined by the formula $d b=1 \otimes b-b \otimes 1=\sum_{i=1}^{n} \partial_{i} b d z_{i}$. As before, one may verify that $\beta_{t}$ is an isomorphism. If $E$ is any bundle on $X$, it is locally the direct sum of line bundles, and one takes a direct sum of $\beta_{t}$ 's to get a trivialization of $\left.\mathrm{P}^{t}(E)\right|_{U}$. The analogue of $(4.6)$ is

$$
\beta_{t}: \Gamma\left(U, \mathrm{P}^{t}(E)\right) \rightarrow\left(A^{\oplus\left(\begin{array}{c}
n+t \\
t
\end{array}\right)}\right)^{\oplus r}
$$

Diagram (4.7) becomes

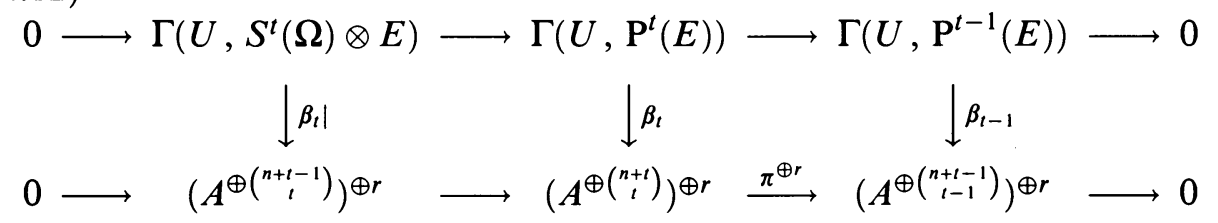

where $\pi$ is the projection onto the first $\left(\begin{array}{c}n+t-1 \\ t-1\end{array}\right)$ factors.

A.5. The bimodule structure on $\mathrm{P}^{t}(\mathscr{F})$ and the map $d^{t}$. Recalling the notation of Definition 1.2, $\mathrm{P}^{t}\left(\mathscr{O}_{X}\right)=p_{*}\left(\mathscr{O}_{\Delta_{(t)}}\right)=p_{*}\left(\mathscr{O}_{X} \otimes \mathscr{O}_{X} / \mathscr{I}^{t+1}\right)$. By definition of $p$, there is a map

$$
\mathscr{O}_{X} \rightarrow p_{*}\left(\mathscr{O}_{\Delta_{(t)}}\right)=\mathrm{P}^{t}\left(\mathscr{O}_{X}\right)
$$

defining the (usual) left module structure on $\mathrm{P}^{t}\left(\mathscr{O}_{X}\right)$. By slight abuse of notation, we write

$$
\begin{aligned}
\mathscr{O}_{X} & \rightarrow\left(\mathscr{O}_{X} \otimes \mathscr{O}_{X}\right) / \mathscr{I}^{t+1}=\mathrm{P}^{t}\left(\mathscr{O}_{X}\right) \\
a & \mapsto a \otimes 1 .
\end{aligned}
$$

On the other hand, we can derive the following map from $q$, ([G, 16.7.5.1]):

$$
\begin{aligned}
d^{t}: \mathscr{O}_{X} & \rightarrow\left(\mathscr{O}_{X} \otimes \mathscr{O}_{X}\right) / \mathcal{F}^{t+1} \\
a & \mapsto 1 \otimes a .
\end{aligned}
$$

This defines the right module structure on $\mathrm{P}^{t}\left(\mathscr{O}_{X}\right)$.

As pointed out in [G, 16.7.2.1], basic properties of pullbacks and pushforwards yield an isomorphism

$$
\mathrm{P}^{t}(\mathscr{F}) \cong \mathrm{P}^{t}\left(\mathscr{O}_{X}\right) \otimes_{\mathscr{O}_{X}} \mathscr{F}
$$

where in order to tensor over $\mathscr{O}_{X}, \mathrm{P}^{t}\left(\mathscr{O}_{X}\right)$ is considered as a right $\mathscr{O}_{X}$-module. Thus, $\mathrm{P}^{t}(\mathscr{F})$ inherits a bimodule structure from $\mathrm{P}^{t}\left(\mathscr{O}_{X}\right)$. One way to see $(5.3)$ is to check-using the notation of (A.1) - that the natural map

$$
\begin{aligned}
\mathrm{P}^{t}\left(\mathscr{O}_{X}\right) \otimes \mathscr{F} & =\pi_{1 *}\left(\left(\mathscr{O}_{X} \otimes \mathscr{O}_{X}\right) / \mathscr{F}^{t+1}\right) \otimes \mathscr{F} \\
& \rightarrow \pi_{1 *}\left(\left(\mathscr{O}_{X} \otimes \mathscr{O}_{X}\right) / \mathscr{F}^{t+1} \otimes \pi_{2}^{*} \mathscr{F}\right)=\mathrm{P}^{t}(\mathscr{F})
\end{aligned}
$$


is an isomorphism. By abuse of notation, we will sometimes write

$$
\mathrm{P}^{t}(\mathscr{F})=\frac{\mathscr{O}_{X} \otimes \mathscr{O}_{X}}{\mathscr{I}^{t+1}} \otimes \mathscr{F}
$$

Define a map ([G, 16.7.5.1]):

$$
\begin{aligned}
d_{\mathscr{F}}^{t}=d^{t}: \mathscr{F} & \rightarrow \mathrm{P}^{t}(\mathscr{F}) \cong \mathrm{P}^{t}\left(\mathscr{O}_{X}\right) \otimes_{\mathscr{O}_{X}} \mathscr{F} \\
s & \mapsto(1 \otimes 1) \otimes s .
\end{aligned}
$$

This is a map of $\mathscr{O}_{X}$-modules if $\mathrm{P}^{t}(\mathscr{F})$ is considered as a right module. The maps $d^{t}$ are compatible with the natural projections $p r_{t}: \mathrm{P}^{t}(\mathscr{F}) \rightarrow \mathrm{P}^{t-1}(\mathscr{F})$, (1.3). That is to say, $p r_{t} \circ d^{t}=d^{t-1}$.

Consider the case of a line bundle $\mathscr{L}$ on a nonsingular curve $X$ over an algebraically closed field. Using the trivialization described in (A.4) with $E=$ $\mathscr{L}$, identify $\mathscr{L}$ with $A=\mathscr{O}_{U}(U)$ and $\mathrm{P}^{t}(\mathscr{L})$ with $A^{\oplus t+1}$ via $\beta_{t},(4.6)$. Then the map $d_{t}$ may be thought of as taking truncated Taylor expansions of sections of $\mathscr{L}$

$$
\begin{aligned}
d^{t}: A & \rightarrow A^{\oplus t+1} \\
a & \mapsto\left(a, \partial_{z} a, \frac{1}{2 !} \partial_{z}^{2} a, \ldots, \frac{1}{t !} \partial_{z}^{t} a\right) .
\end{aligned}
$$

A.6. The Taylor series map, $\nu^{t}$. With the notation of A.1, the natural maps $u^{*} u_{*} \rightarrow \pi_{2 *} \pi_{1}^{*}$ and $1 \rightarrow i_{*} i^{*}$ may be used to define what we will call the $t$-th Taylor series map, (cf. [K1], [Pi1]):

$$
\nu^{t}: u^{*} u_{*} \mathscr{F} \rightarrow \pi_{1 *} \pi_{2}^{*} \mathscr{F} \longrightarrow \pi_{1 *} i_{*} i^{*} \pi_{2}^{*} \mathscr{F} \cong \mathrm{P}^{t}(\mathscr{F}) .
$$

If $V$ is a sheaf on $S$ and $\phi: u^{*} V \rightarrow \mathscr{F}$ any map, then there is a natural map

$$
V_{X}=u^{*} V \rightarrow u^{*} u_{*} u^{*} V \rightarrow u^{*} u_{*} \mathscr{F} \text {. }
$$

Composing this with the Taylor series map yields a map $V_{X}=u^{*} V \rightarrow \mathrm{P}^{t}(\mathscr{F})$ which will also be called a Taylor series map and be denoted by $\nu_{\phi}^{t}$ or just $\nu^{t}$ if $\phi$ is clear from context. If we consider $\mathrm{P}^{t}(\mathscr{F})$ as a left $\mathscr{O}_{X}$-module (as usual), then $\nu^{t}$ is an $\mathscr{O}_{X}$-module map.

If $X$ is a noetherian scheme and $S=\operatorname{Spec} B$, then the Taylor series map is

$$
\nu^{t}: \mathrm{H}^{0}(X, \mathscr{F}) \otimes_{B} \mathscr{O}_{X} \rightarrow \mathrm{P}^{t}(\mathscr{F})
$$

(cf. $[\mathrm{H}, 8.5])$. With the notation of (A.5), we may write this as

$$
\begin{aligned}
\mathrm{H}^{0}(X, \mathscr{F}) \otimes_{B} \mathscr{O}_{X} & \rightarrow \mathrm{P}^{t}(\mathscr{F}) \cong \mathrm{P}^{t}\left(\mathscr{O}_{X}\right) \otimes_{\mathscr{O}_{X}} \mathscr{F} \\
s \otimes 1 & \mapsto 1 \otimes 1 \otimes s .
\end{aligned}
$$

Therefore, we can also construct $\nu^{t}$ by taking global sections of $d^{t}: \mathscr{F} \rightarrow \mathrm{P}^{t}(\mathscr{F})$ and then evaluating

$$
\mathrm{H}^{0}(X, \mathscr{F}) \otimes_{B} \mathscr{O}_{X} \stackrel{d^{t}}{\longrightarrow} \mathrm{H}^{0}\left(X, \mathrm{P}^{t}(\mathscr{F})\right) \otimes_{B} \mathscr{O}_{X} \rightarrow \mathrm{P}^{t}(\mathscr{F}) .
$$

\subsection{Functorial properties of the Taylor series map.}

6.3.1. The Taylor series map preserves direct sums since pullbacks, pushforwards, and the map $1 \rightarrow i_{*} i^{*}$ preserve direct sums. 
6.3.2. The Taylor series map is compatible with the natural surjections $\mathrm{P}^{t}(\mathscr{F})$ $\rightarrow \mathrm{P}^{t-1}(\mathscr{F})$. To see this, let $i_{k}: \Delta_{(k)} \rightarrow X \times_{S} X$ denote the inclusion of the thickened diagonal (cf. A.1). The compatibility of the Taylor series map comes from applying $\pi_{1 *}(\cdot) \pi_{2}^{*}$ to the natural commutative diagram of functors

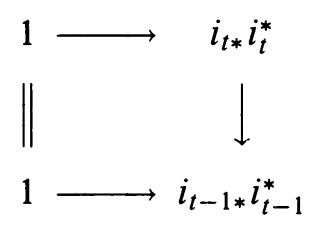

and composing with the map $u^{*} u_{*}$ of (6.1).

6.3.3. If $\mathscr{F} \rightarrow \mathscr{G}$ is a map of sheaves on $X$, the naturality of the map $u^{*} u_{*} \rightarrow \pi_{1 *} \pi_{1}^{*}$ gives a commutative diagram

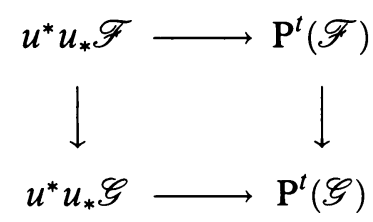

where the horizontal maps are the Taylor series maps and the vertical maps are the natural ones.

In the paper, (6.3.3.1) is most often used in the following form:

Proposition 6.3.3.2. Let $V$ and $W$ be sheaves on $S$, and let $\mathscr{F}$ and $\mathscr{G}$ be sheaves on $X$. Suppose we are given a commutative diagram of sheaves on $X$ :

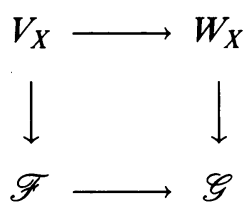

Then the following diagram commutes

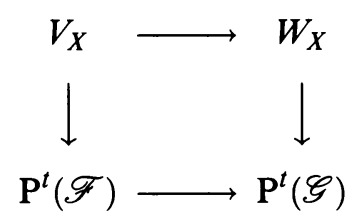

where the vertical maps are the Taylor series maps and the horizontal maps are the natural ones.

Proof. This follows immediately from the expression for $\nu^{t}$ given in (6.2).

6.3.4. Let $S_{E}$ be the kernel of the map $V_{X} \rightarrow \mathscr{F}$ from Proposition 6.3.3.2. Assume in addition that $X \rightarrow S$ is smooth and $\mathscr{F}$ is locally free. The compatibility of the Taylor series map with the standard projections, (6.3.2), gives a commutative diagram that is used repeatedly in the paper:

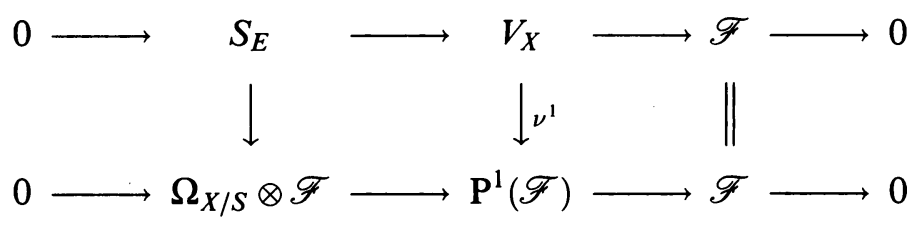


The bottom row of this diagram is the fundamental exact sequence (3.2). This diagram is functorial in $\mathscr{F}$ :

Proposition 6.3.4.2. With the notation as in (6.3.3.2), assume in addition that $X \rightarrow S$ is smooth and that $\mathscr{F}$ and $\mathscr{G}$ are locally free. Let $S_{\mathscr{F}}$ (respectively, $\left.S_{\mathscr{G}}\right)$ denote the kernel of the given map $V_{X} \rightarrow \mathscr{F}$ (respectively, $W_{X} \rightarrow \mathscr{G}$ ). Consider the natural commutative diagrams with exact rows

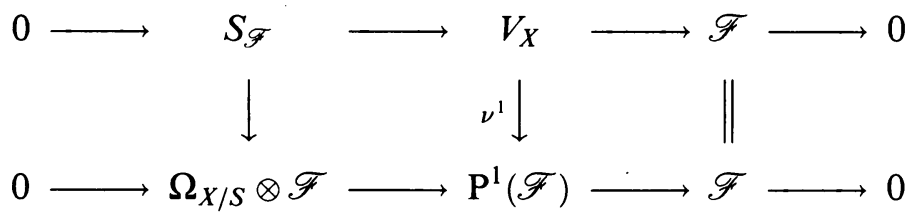

and

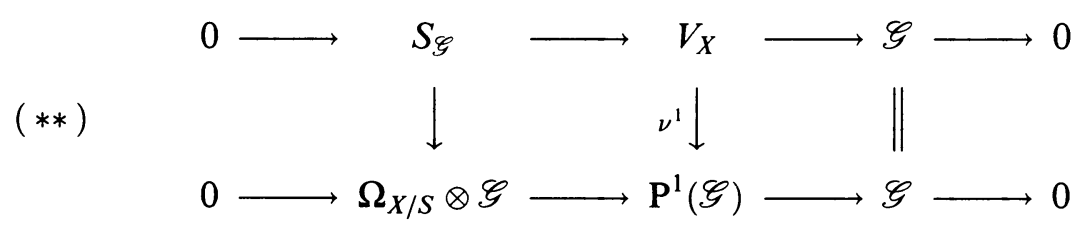

where the bottom rows of these diagrams are the fundamental exact sequences of (A.3). Proposition 3.4 induces a natural map of commutative diagrams $(*) \rightarrow$ $(* *)$.

6.3.5. Taylor series maps are compatible with the maps of $(2.4), f^{*} \mathrm{P}^{t}(\mathscr{F}) \rightarrow$ $\mathrm{P}^{t-1}(\mathscr{F})$. This is immediate from the construction of the maps of (2.4), given in the proof of Proposition 3.4.

6.4. Description of the Taylor series map in local coordinates. $u: X \rightarrow S$ be smooth of some relative dimension, $\mathscr{F}$ be locally free of rank $r$, and $V$ be a locally free $\mathscr{O}_{S}$-module of rank $n$. Assume we are given a map

$$
\phi: V_{X}=u^{*} V \rightarrow \mathscr{F} \text {. }
$$

We now want to give a careful local description of $\nu^{t}(\phi)=\nu^{t}$. So we may assume $X$ and $S$ are rings, $A$ and $B$, respectively, and that $\mathscr{F}=A^{\oplus r}, V_{X}=$ $A^{\oplus n}$, and $\phi$ is given by a matrix $M=\left(a_{i j}\right): A^{\oplus n} \rightarrow A^{\oplus r}$. Define $\mathrm{P}^{t}\left(A^{\oplus r}\right)=$ $\Gamma\left(X, \mathrm{P}^{t}(\mathscr{F})\right)$, and define $\nu^{t}$ accordingly. Let $d: A \rightarrow \Omega_{A / B}$ be the standard derivation. Our smoothness assumption means that $\Omega_{A / B}$ is a free $A$-module with generators, say, $d z_{i}$. Define the map

$$
\partial_{z_{i}}^{j}: A \longrightarrow A
$$

inductively as follows

$$
d a=\sum \partial_{z_{i}} a d z_{i}, \quad \partial_{z_{i}}^{j} a=\partial_{z_{i}}\left(\partial_{z_{i}}^{j-1} a\right) .
$$

Note 6.4.2. If $A$ is a discrete valuation ring and $B=k$ is a field isomorphic to the residue field of $A$, then the completion of $A$ is isomorphic to the power series ring $k[[z]]$. Emdedding $A$ into its completion, $\partial_{z}^{j} a_{i}$ can be thought of as the ordinary $j$-th derivative of a power series. This interpretation will be relevant when $X$ is a smooth curve over an algebraically closed field and we consider a Taylor series map at a fiber over a point in $X$. 
To make the local description more intelligible, we will start out with the easy case of relative dimension and $r$ both equal to one, then work our way up to the general case.

Proposition 6.4.3. Let $B \rightarrow A$ be a map of rings with the characteristic of each residue field of $A$ and $B$ either zero or greater than $t$. Assume $\Omega_{A / B}$ has rank one, generated by the single element $d z$, and suppose given a map

$$
M: A^{\oplus n} \rightarrow A
$$

where $M=\left[a_{1}, \ldots, a_{n}\right], a_{i} \in A$. Then the corresponding $t$-th Taylor series map has the form

$$
\nu^{t}(M): A^{\oplus n} \stackrel{\left[\begin{array}{ccc}
a_{1} & \cdots & a_{n} \\
\partial_{z} a_{1} & \cdots & \partial_{z} a_{n} \\
\frac{1}{2 !} \partial_{z}^{2} a_{1} & \cdots & \frac{1}{2 !} \partial_{z}^{2} a_{n} \\
\vdots & \cdots & \vdots \\
\frac{1}{t !} \partial_{z}^{t} a_{1} & \cdots & \frac{1}{t !} \partial_{z}^{t} a_{n}
\end{array}\right]}{\longrightarrow} \mathrm{P}^{t}(A) \cong A^{\oplus t+1} .
$$

Proof. Let $e_{i}$ be the $i$-th standard basis vector for $A^{\oplus n}$. Define $I$ as in (A.4) so that $\Omega_{A / B}=I / I^{2}$. By the description in (6.2),

$$
\begin{aligned}
\nu^{t}\left(e_{i}\right) & =(1 \otimes 1) \otimes a_{i} \in(A \otimes A) / I^{t+1} \otimes A \\
& =1 \otimes a_{i} \in(A \otimes A) / I^{t+1}=\mathrm{P}^{t}(A) .
\end{aligned}
$$

Using $\beta_{t}$ of (4.2) to trivialize $\mathrm{P}^{t}(A)$ yields

as required.

$$
\nu^{t}\left(e_{i}\right)=\left(a_{i}, \partial_{z} a_{i}, \frac{1}{2 !} \partial_{z}^{2} a_{i}, \ldots, \frac{1}{t !} \partial_{z}^{t} a_{i}\right)
$$

We will now consider the case where $X \rightarrow S$ has relative dimension one but $r$, the rank of $\mathscr{F}$, is arbitrary. Locally, $\mathscr{F} \cong A^{\oplus r}$, and the local description of the Taylor series map follows directly from Proposition 6.4.3.

Proposition 6.4.4. Let $B \rightarrow A$ be a map of rings with the characteristic of each residue field of $A$ and $B$ either zero or greater than $t$. Assume $\Omega_{A / B}$ has rank one, generated by the single element $d z$, and suppose given a map

$$
M: A^{\oplus n} \longrightarrow A^{\oplus r}
$$

where $M=\left(a_{i j}\right), a_{i j} \in A$. Then the corresponding $t$-th Taylor series map is a block matrix

$$
\nu^{t}(M): A^{\oplus n} \stackrel{\left[\begin{array}{c}
M \\
\partial_{z} M \\
\frac{1}{2 !} \partial_{z}^{2} M \\
\vdots \\
\frac{1}{t !} \partial_{z}^{t} M
\end{array}\right]}{\longrightarrow} \mathbf{P}^{t}\left(A^{\oplus r}\right) \cong\left(A^{\oplus r}\right)^{\oplus t+1}
$$

where $\partial_{z}^{k} M=\left(\partial_{z}^{k} a_{i j}\right)_{i, j}$.

Proof. This follows directly from Proposition 6.4.1 and the fact that $\nu^{t}$ preserves direct sums, (6.3.1). 
Finally, we consider the case of arbitrary relative dimension. It is the same as (6.4.4) except we must take all mixed partials of the entries of $\phi$ with respect to local parameters for $X$.

Proposition 6.4.5. Let $B \rightarrow A$ be a map of rings with the characteristic of each residue field of $A$ and $B$ either zero or greater than $t$. Assume $\Omega_{A / B}$ has rank $k$, generated by $d z_{1}, \ldots, d z_{k}$, and suppose given a map

$$
M: A^{\oplus n} \rightarrow A^{\oplus r}
$$

where $M=\left(a_{i j}\right), a_{i j} \in A$. Then the corresponding $t$-th Taylor series map is a block matrix

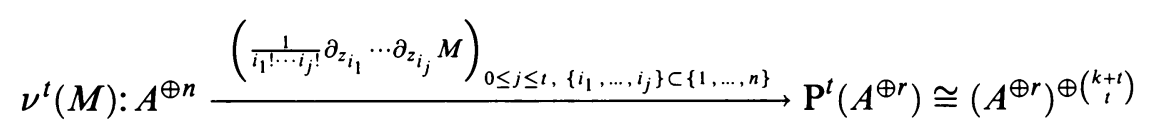

where $\partial_{z_{k_{1}}} \cdots \partial_{z_{k_{\ell}}} M=\left(\partial_{z_{k_{1}}} \cdots \partial_{z_{k_{\ell}}} a_{i j}\right)_{i, j}$. Each $\left(\frac{1}{i_{1} ! \cdots i_{j} !} \partial_{i_{1}} \cdots \partial_{i_{j}} M\right)$ is a row of the block matrix $\nu^{t}(M)$.

Proof. For the case $r=1$, proceed as in the proof to Proposition 6.4.1 using the map $\beta_{t}$ of (4.10). (The $n$ of (4.10) is our $k$.) Then, for general $r$, use the fact that $\nu^{t}$ preserves direct sums, (6.3.1).

A.7. $\mathrm{P}^{n+n^{\prime}}(\mathscr{F}) \rightarrow \mathrm{P}^{n}\left(\mathrm{P}^{n^{\prime}}(\mathscr{F})\right)$. There is a natural map, ([G, 16.8.9.1]),

$$
\delta=\delta_{n, n^{\prime}}: \mathrm{P}^{n+n^{\prime}}(\mathscr{F}) \rightarrow \mathrm{P}^{n}\left(\mathrm{P}^{n^{\prime}}(\mathscr{F})\right)
$$

functorial in $\mathscr{F}$, making the following diagram commute

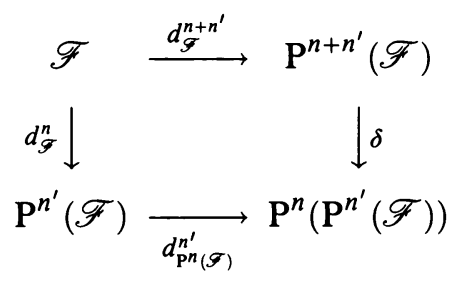

Using (5.3) and the notation of (A.5), the map is given by (7.3)

$$
\begin{aligned}
\mathrm{P}^{n+n^{\prime}}(\mathscr{F})= & \frac{\mathscr{O}_{X} \otimes \mathscr{O}_{X}}{\mathcal{I}^{n+n^{\prime}+1}} \otimes \mathscr{F} \rightarrow \frac{\mathscr{O}_{X} \otimes \mathscr{O}_{X}}{\mathscr{I}^{n+1}} \otimes \frac{\mathscr{O}_{X} \otimes \mathscr{O}_{X}}{\mathscr{I}^{n^{\prime}+1}} \otimes \mathscr{F}=\mathrm{P}^{n}\left(\mathrm{P}^{n^{\prime}}(\mathscr{F})\right), \\
(a \otimes b) \otimes f & (a \otimes 1) \otimes(1 \otimes b) \otimes f .
\end{aligned}
$$

(As in (A.5), we are abusing notation slightly.) As noted after (5.3), we must be careful of the module structure when taking these tensors. Thus,

$$
(a \otimes b) \otimes c f=(a \otimes c b) \otimes f \in\left(\mathscr{O}_{X} \otimes \mathscr{O}_{X}\right) / \mathscr{I}^{n+n^{\prime}+1} \otimes \mathscr{F}
$$

and

$(a \otimes b) \otimes(c \otimes d) \otimes e f=(a \otimes b c) \otimes(1 \otimes d e) \otimes f$

$$
=(a \otimes 1) \otimes(b c \otimes d e) \otimes f \in \frac{\mathscr{O}_{X} \otimes \mathscr{O}_{X}}{\mathscr{I}^{n+1}} \otimes \frac{\mathscr{O}_{X} \otimes \mathscr{O}_{X}}{\mathscr{I}^{\prime}+1} \otimes \mathscr{F} .
$$

Here $a, b, c, d, e$ (respectively, $f$ ) represent sections of $\mathscr{O}_{X}$ (respectively, $\mathscr{F}$ ) over some open set of $X$. 
We will need to know that $\delta$ is compatible with the Taylor series map. Consider the Taylor series maps

$$
u^{*} u_{*} \mathscr{F} \rightarrow \mathrm{P}^{n^{\prime}}(\mathscr{F})
$$

and

$$
u^{*} u_{*} \mathrm{P}^{n^{\prime}}(\mathscr{F}) \longrightarrow \mathrm{P}^{n}\left(\mathrm{P}^{n^{\prime}}(\mathscr{F})\right) .
$$

Applying $u^{*} u_{*}$ to the first of these maps and using the natural map $1 \rightarrow u_{*} u^{*}$ gives the left vertical map in the following diagram

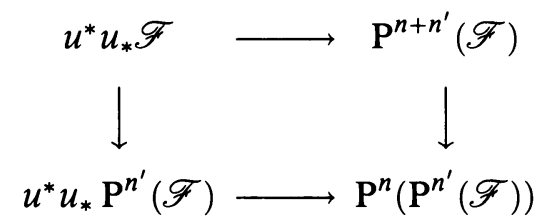

The horizontal maps are Taylor series maps. Using (7.3), it is clear that this diagram commutes, i.e., $\delta$ is compatible with Taylor series maps.

It also clear from (7.3) that $\delta$ commutes with the natural surjections from $t$ jets to $(t-1)$-jets in several senses. For example, there are natural commutative diagrams:

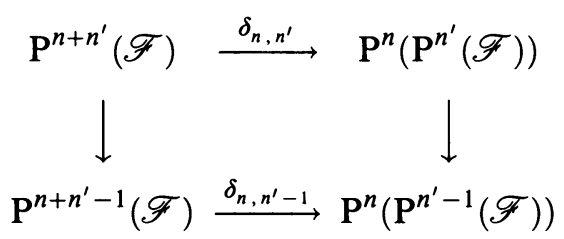

and

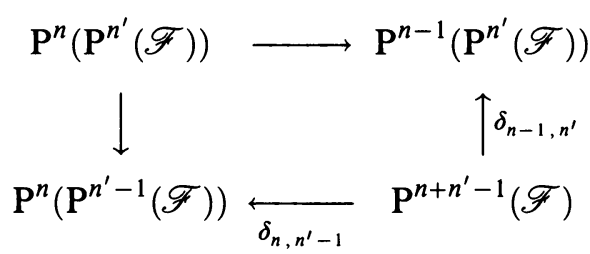

Remark 7.7. Letting $n=1,(7.6)$ shows that $\mathrm{P}^{1}\left(\mathrm{P}^{n^{\prime}}(\mathscr{F})\right) \rightarrow \mathrm{P}^{1}\left(\mathrm{P}^{n^{\prime}-1}(\mathscr{F})\right)$ factors through the surjection $\mathrm{P}^{1}\left(\mathrm{P}^{n^{\prime}}(\mathscr{F})\right) \rightarrow \mathrm{P}^{n^{\prime}}(\mathscr{F})$.

We will need the following technical result:

Proposition 7.8. Assume that the characteristic of $S$ is zero or greater than $n+1$. If $X \rightarrow S$ is smooth of relative dimension one, and $\mathscr{F}$ is a locally free sheaf on $X$, then the map $\delta: \mathrm{P}^{n+1}(\mathscr{F}) \rightarrow \mathrm{P}^{1}\left(\mathrm{P}^{n}(\mathscr{F})\right)$ is an inclusion with a locally free cokernel.

Proof. The compatibility of $\delta$ with the natural surjections of principal parts bundles gives a commutative diagram (7.8.1)

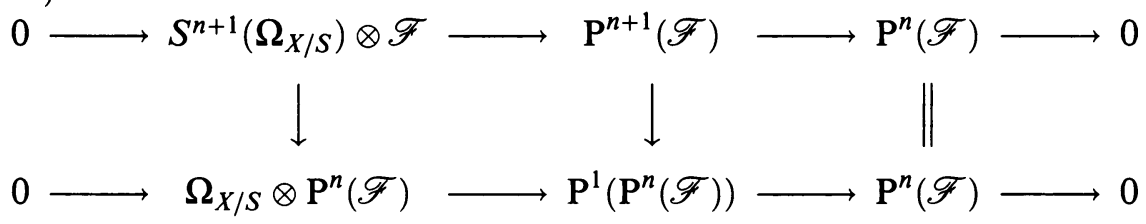


We will now show that since $X \rightarrow S$ has relative dimension one, that the leftmost vertical map in (7.8.1) becomes $\Omega_{X / S} \otimes \Omega_{X / S}^{\otimes n} \otimes \mathscr{F} \stackrel{\mu \otimes i}{\longrightarrow} \Omega_{X / S} \otimes \mathrm{P}^{n}(\mathscr{F})$ where $i: \mathscr{F} \otimes \Omega^{n} \rightarrow \mathrm{P}^{n}(\mathscr{F})$ denotes the natural inclusion of (3.2), and $\mu: \Omega_{X / S} \rightarrow$ $\Omega_{X / S}$ is multiplication by $(n+1)$. In particular, $\mu \otimes i$ is injective; hence the proposition follows from the snake lemma.

By (7.3) we may write

$$
\begin{gathered}
\delta: \frac{\mathscr{O}_{X} \otimes \mathscr{O}_{X}}{\mathscr{I}^{n+2}} \otimes \mathscr{F} \rightarrow \frac{\mathscr{O}_{X} \otimes \mathscr{O}_{X}}{\mathscr{I}^{2}} \otimes \frac{\mathscr{O}_{X} \otimes \mathscr{O}_{X}}{\mathscr{I}^{n+1}} \otimes \mathscr{F}, \\
(a \otimes b) \otimes f \mapsto(a \otimes 1) \otimes(1 \otimes b) \otimes f .
\end{gathered}
$$

We need to find the image of

$$
d z^{n+1} \otimes f \in\left(\mathscr{I}^{n+1} / \mathscr{I}^{n+2}\right) \otimes \mathscr{F}=\Omega_{X / S}^{\otimes n+1} \otimes \mathscr{F} .
$$

Calculate

$$
\begin{aligned}
\delta & \left(d z^{n+1} \otimes f\right)=\delta(1 \otimes z-z \otimes 1)^{n+1} \otimes f \\
& =[(1 \otimes 1) \otimes(1 \otimes z)-(z \otimes 1) \otimes(1 \otimes 1)]^{n+1} \otimes f \\
& =[d z \otimes(1 \otimes 1)+(1 \otimes 1) \otimes d z]^{n+1} \otimes f \\
& =\left(\sum_{k=0}^{n+1}\left(\begin{array}{c}
n+1 \\
k
\end{array}\right) d z^{k} \otimes d z^{n+1-k}\right) \otimes f \\
& =(n+1) d z \otimes d z^{n} \otimes f
\end{aligned}
$$

Thus, the leftmost vertical map of (7.8.1) is as required.

A.8. Osculating bundles. The following definition is due to Piene, [Pi1].

Definition 8.1. Let $V$ be a sheaf on $S$, let $\mathscr{F}$ be a sheaf on $X$, and let $\phi: V_{X} \rightarrow \mathscr{F}$ be a map of sheaves. For $t \geq 0$, the image of the Taylor series map $\nu_{\phi}^{t}: V_{X} \rightarrow \mathrm{P}^{t}(\mathscr{F})$ is called the osculating sheaf of order $t$ for $\phi$ and denoted by $\mathrm{G}^{t}(\phi)$ or just $\mathrm{G}^{t}(\mathscr{F})$ when $\phi$ is clear from context. It comes with a natural surjection

$$
\mu^{t}: V_{X} \rightarrow \mathrm{G}^{t}(\mathscr{F})
$$

The natural surjections, $\mathrm{P}^{t}(\mathscr{F}) \rightarrow \mathrm{P}^{t-1}(\mathscr{F})$, induce surjections, $\mathrm{G}^{t}(\mathscr{F}) \rightarrow$ $\mathrm{G}^{t-1}(\mathscr{F})$.

Proposition 8.2. Let $V, W$ be sheaves on $S$, and let $\mathscr{F}, \mathscr{G}$ be sheaves on $X$. Suppose there is a commutative diagram

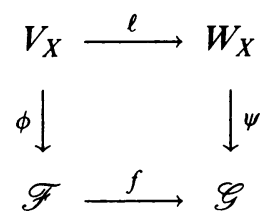


Then there are maps between osculating sheaves $f_{t}: G^{t}(\mathscr{F}) \rightarrow G^{t}(\mathscr{G})$ such that

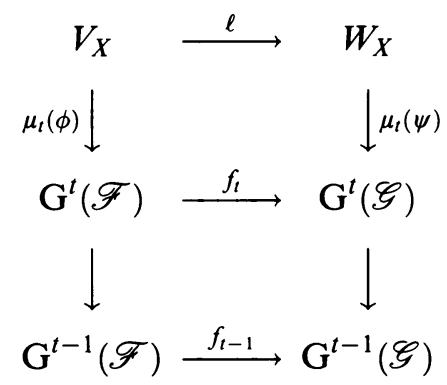

commutes for $t \geq 1$. The bottom vertical maps are the natural surjections, and the composition of the vertical maps on the left (respectively, right) is $\mu_{t-1}(\phi)$ (respectively, $\mu_{t-1}(\psi)$ ).

For (2) and (3) below, assume that $\mathscr{F}$ and $\mathscr{G}$ are locally free.

(1) If $\ell$ is surjective, then so are the $f_{t}$.

(2) If $f$ is injective, so are the $f_{t}$.

(3) If $f$ is injective and $\ell$ is surjective, then the $f_{t}$ are isomorphisms;

Proof. The maps $\ell$ and $f$ give rise to a commutative diagram, (6.3.3.2)

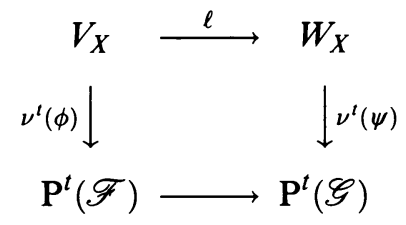

Taking images of the vertical maps defines $f_{t}$. The required compatibility with the natural surjections follows from the corresponding fact for principal parts of sheaves, (6.3.2).

(1) is clear from the definition of $f_{t}$.

Restricted to locally free sheaves, $\mathrm{P}^{t}(\cdot)$ is an exact functor (2.3). This accounts for (2), and as a trivial consequence of (1) and (2), we get (3).

Proposition 8.3. Let $\mathscr{F}$ be locally free. There is a surjection

$$
\mathrm{G}^{t}(\mathscr{F}) \rightarrow \mathrm{G}^{1}\left(\mathrm{G}^{t-1}(\mathscr{F})\right)
$$

compatible with the natural surjections from $V_{X}$. It is functorial in $\mathscr{F}$ and is compatible with the natural surjections of osculating bundles, i.e., there is a commutative diagram

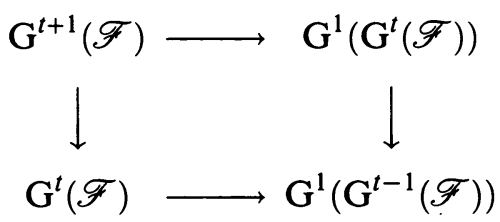

If $X \rightarrow S$ is smooth of relative dimension one and the characteristic of each residue field of $S$ is zero or greater than $t$, then (*) is an isomorphism.

Proof. By (2.3), applying $\mathrm{P}^{1}(\cdot)$ to the inclusion $\mathrm{G}^{t-1}(\mathscr{F}) \hookrightarrow \mathrm{P}^{t-1}(\mathscr{F})$ yields

$$
\mathrm{G}^{1}\left(\mathrm{G}^{t-1}(\mathscr{F})\right) \hookrightarrow \mathrm{P}^{1}\left(\mathrm{G}^{t-1}(\mathscr{F})\right) \hookrightarrow \mathrm{P}^{1}\left(\mathrm{P}^{t-1}(\mathscr{F})\right) .
$$


On the other hand, we have

$$
\mathrm{G}^{t}(\mathscr{F}) \hookrightarrow \mathrm{P}^{t}(\mathscr{F}) \stackrel{\delta}{\longrightarrow} \mathrm{P}^{1}\left(\mathrm{P}^{t-1}(\mathscr{F})\right)
$$

where $\delta$ is the map of (7.1). Since these maps are compatible with the natural surjections from $V_{X}, \mathrm{G}^{t}(\mathscr{F})$ surjects onto the image of $\mathrm{G}^{1}\left(\mathrm{G}^{t-1}(\mathscr{F})\right)$ in $\mathrm{P}^{1}\left(\mathrm{P}^{t-1}(\mathscr{F})\right)$. This gives $(*)$. With the additional assumption on $X \rightarrow S$ and the characteristic of $S$, it follows from Proposition 7.8 that $\delta$ in ( $\ddagger)$ is injective. Therefore, in that case, $(*)$ is an isomorphism.

The functoriality of $(*)$ comes from that of $\mathrm{P}^{t}(\cdot)$ and of $\delta$. Compatibility with the surjections of osculating bundles follows from (7.5).

\section{APPENDIX B}

Introduction. This appendix is an outline of the basic theory of inflections of curves in projective space. It is intended as background and a convenient reference. For the most part, results are presented without proofs. The main theorems are due to Piene, and details may be found in [Pi1] and [Pe1]. The two most fundamental theorems in the theory are Theorem 2.3, stating the degrees and ranks of the osculating bundles, and the duality theorem in (B.5).

Throughout the appendix, $V$ denotes a vector space of dimension $n+1$ over an algebraically closed field $k$, and $X$ is a smooth curve over $k$. Let

$$
f: X \rightarrow \mathbb{P}(V) \cong \mathbb{P}_{k}^{n}
$$

be a map to the projective space of quotients of $V$. There is a corresponding surjection

$$
\phi: V_{X} \rightarrow \mathscr{L}
$$

where $\mathscr{L}$ is a line bundle on $X$. The map on global sections will be denoted

$$
\Gamma_{\phi}: V \rightarrow \Gamma(X, \mathscr{L})
$$

Definition 0.4. If $m$ is the dimension of the smallest linear space containing the image of $f$, then $f$ is said to span a $\mathbb{P}^{m}$ or span a linear space of dimension $m$. The number $m$ is one less than the dimension of the image of $\Gamma_{\phi}$.

B.1. Inflection numbers. In this section, we introduce the fundamental invariants describing the inflectionary behavior of a curve in projective space. This material can also be found in [GH1], [Pi1], and [L1].

Definition 1.1. Let $f$ span a $\mathbb{P}^{m}$. For each $x \in X$, define integers $\alpha_{i}=\alpha_{i}(x)$ with $0=\alpha_{0} \leq \alpha_{1} \leq \cdots \leq \alpha_{m}$ by

$$
\left\{0,1+\alpha_{1}, 2+\alpha_{2}, \ldots, m+\alpha_{m}\right\}=\left\{\operatorname{ord}_{x}(\sigma)\right\}_{\sigma \in \Gamma_{\phi}(V)} .
$$

(A Gram-Schmidt-type argument shows that this definition yields $m$ numbers.) The number $\alpha_{i}$ is called the $i$-th inflection number for $\phi$ (or $f$ ) at $x$. If some $\alpha_{i}$ is nonzero, $x$ is said to be an inflectionary point for $\phi$ at $x$. Under appropriate conditions, $(2.3)$, there will be a finite number of inflectionary points. In this case, it makes sense to sum an $\alpha_{i}$ over all points to get a global $i$-th inflection number for $\phi$, also denoted by $\alpha_{i}$, and we define the $i$-th inflection 
divisor to be $\sum_{x \in X} \alpha_{i}(x) \cdot x$. Later we will define a related inflection sheaf, (2.4).

Inflectionary points are also called points of hyperosculation since, at each of these points, an osculating space meets $f(X)$ with higher multiplicity than expected, (4.1.1).

Remark 1.2. Let $\mathscr{L}=\mathscr{O}(D)$ for a divisor $D$ on $X$. For $x \in X$ consider the decreasing sequence of integers

$$
\ell_{x}(n)=\operatorname{dim}_{k}\left\{\sigma \in \Gamma_{\phi}(V) \mid \sigma \in \Gamma(X, \mathscr{O}(D-n x))\right\}
$$

for $n=0,1,2, \ldots$. If $\ell_{x}(n-1) \neq \ell_{x}(n)$, then $n$ is called a gap value for $\phi$ at $x$. (Note: If $\ell_{x}(n-1) \neq \ell_{x}(n)$, then $\ell_{x}(n-1)=\ell_{x}(n)-1$, (cf. [H, proof of Proposition IV.3.1]).) If the gap values are not $1,2, \ldots, m$, then the point $x$ is called a generalized Weierstrass point. Denote the $i$-th gap value by $a_{i}$. The Weierstrass weight for $\phi$ at $x$ is the integer $\sum_{i=1}^{m}\left(a_{i}-i\right)$. In terms of the inflectionary indices, the $i$-th gap value is $\alpha_{i-1}+i$. The classical situation is when the genus of $X$ is greater than one and $\phi: V \stackrel{\equiv}{\Longrightarrow}\left(X, \Omega_{X / k}\right) \rightarrow \Omega_{X / k},(3.2)$; the gap values measure the inflectionary behavior of the canonical embedding.

1.3. Normal form. By a normal form for the map $f$ at $x$ we mean a choice of coordinates for $\mathbb{P}^{n}$ that is nice with respect to the inflection numbers. Specifically, choose a basis, $\sigma_{0}, \ldots, \sigma_{m}$, for the image of $\phi$ such that $\operatorname{ord}_{x}\left(\sigma_{i}\right)=i+\alpha_{i}$. Identify $\mathscr{L}_{x}$ with the local ring at $x, A=\mathscr{O}_{X, x}$, and let $z$ be a local parameter at $x$. We can think of the $\sigma_{i}$ 's as elements in the completion $\hat{A} \cong k[[z]]$, so that $f$ is given parametrically by

$$
z \mapsto v(z)=\left(1+\cdots, z^{1+\alpha_{1}}+\cdots, \ldots, z^{m+\alpha_{m}}+\cdots, 0, \ldots, 0\right)
$$

where " $+\cdots$ " denotes the sum of terms of higher order in $z$. Thus, if $\alpha_{1}>0$, then $f(x)$ is a cusp of the image of $f$, and if $\alpha_{1}=0$ but $\alpha_{2}>0$, then $f(x)$ is an inflection point of the image of $f$.

Let $x_{0}, \ldots, x_{n}$ be coordinates on $\mathbb{P}^{n}$. The expression (1.3.1) shows that the $t$-plane $\left\{x_{t+1}=0, \ldots, x_{n}=0\right\}$ meets $f(x)$ with multiplicity $t+1+\alpha_{t+1}$ along the branch of $f(X)$ corresponding to $x$.

1.4. Base points. We may also define inflection numbers for arbitrary linear systems or for any map (i.e., not necessarily surjective)

$$
V_{X} \rightarrow \mathscr{L} \text {. }
$$

The only difference is that the smallest inflection number $\alpha_{0}$ may no longer be zero. If $\mathscr{L}=\mathscr{O}(D)$ is the line bundle corresponding to a divisor $D$, and $B$ is the base of the linear system corresponding to $(*)$, then the map in $(*)$ factors as

$$
V \stackrel{\psi}{\longrightarrow} \Gamma(X, \mathscr{O}(D-B)) \hookrightarrow \Gamma(X, \mathscr{O}(D)) .
$$

Letting $\beta_{i}(x)$ denote the inflection numbers (or divisors) for the surjection, $\psi$, it is clear from the normal form, (1.3), that

$$
\beta_{i}(x)= \begin{cases}\alpha_{i}, & \text { if } x \text { is not a base point } \\ \alpha_{i}(x)-\alpha_{0}(x), & \text { if } x \text { is a base point. }\end{cases}
$$


B.2. Principal parts. The main result of this section is Theorem 2.3, stating the degree and rank of Piene's osculating bundles. It leads directly to the generalized Plücker formulas, (3.3), and the degrees of varieties associated with the flexing of a curve in space, (B.4).

For each integer $t \geq 0$, the map $\phi$ of $(0.2)$ can be lifted to the $t$-th order Taylor series map, (A.6),

$$
\nu^{t}=\nu_{\phi}^{t}: V_{X} \rightarrow \mathrm{P}^{t}(\mathscr{L})
$$

where $\mathrm{P}^{t}(\mathscr{L})$ is the bundle of $t$-th order jets of sections of $\mathscr{L}$. Locally, we can think of $\phi$ as given by a $n+1$-tuple of functions in a local parameter for the curve. The map $\nu^{t}$ can then be thought of as a matrix with rows consisting of the derivatives of $\phi$ up to order $t$, (A.6.4).

Recall from (A.8) that the image of $\nu^{t}$ is the $t$-th osculating bundle for $\phi$, denoted by $\mathrm{G}^{t}(\mathscr{L})$ (or by $\mathrm{G}^{t}(\phi)$ or $\mathrm{G}^{t}(f)$, if necessary). From $\nu^{t}$, there is a natural surjection

$$
\mu^{t}: V_{X} \rightarrow \mathrm{G}^{t}(\mathscr{L}) .
$$

( $\mathrm{G}^{t}(\mathscr{L})$ is a bundle since it is a subsheaf of $\mathrm{P}^{t}(\mathscr{L})$ and, hence, is torsion free.)

The following theorem, due to Piene [Pil], (with a slight correction due to Laksov, [L1]), shows how the Taylor series maps, $\nu^{t}$, are related to the inflection numbers defined in (B.1). It is the main result from Appendix B needed in the main body of the paper.

Theorem 2.3. Let $X$ be a smooth projective curve, and assume the characteristic of $k$ is zero or greater than $t$ and the degree of $\mathscr{L}$. Then

(1) If $t \leq m=\operatorname{dim}_{k} \Gamma_{\phi}(V)-1$, then $\nu^{t}$ is generically surjective with

$$
\text { length }\left(\operatorname{cok} \nu_{x}^{t}\right)=\sum_{i=1}^{t} \alpha_{i}(x) \text {. }
$$

In particular, $\mathrm{rkG}^{t}(\mathscr{L})=t+1$, and $\nu^{t}$ is surjective if and only if $\phi$ is noninflectionary at $x$ up to order $t$.

(2) There is a finite number of inflectionary points.

(3) If $t \geq m$, then the image is a trivial bundle of rank $m+1$, i.e., $\operatorname{im} \nu^{t}=$ $\mathrm{G}^{t}(\mathscr{L})=\Gamma_{\phi}(V)_{X}$.

Remark 2.3.1. To see that the assumption on the characteristic is necessary in Theorem 2.3 and in Corollary 2.5.3, below, see Remark 3.4.2.

Remark 2.3.2. If $X$ is a smooth projective curve, then with no assumption on the characteristic of $k$,

$$
\alpha_{i}(x) \leq \operatorname{deg} \mathscr{L}-i
$$

for each $x \in X$. Since $\alpha_{i}(x) \leq a_{i+1}(x)$, this means that

$$
a_{i}(x) \leq \operatorname{deg} \mathscr{L}-m
$$

for $i=1, \ldots, m$. This inequality is sharp. For instance, consider the map

$$
\begin{aligned}
\mathbb{P}^{1} & \rightarrow \mathbb{P}^{m} \\
z & \mapsto\left(1, z^{1+(d-m)}, z^{2+(d-m)}, \ldots, z^{m+(d-m)}\right)
\end{aligned}
$$


given by sections of the line bundle $\mathscr{O}(d)$. Here, $\alpha_{i}=d-m$ for all $i$.

Remark 2.3.3. The projectivity assumption can be replaced with the condition that if the characteristic of $k$ is not zero, then it is larger than $m$ and $\alpha_{m}+m$.

Definition 2.4. From the natural surjection of principal parts bundles, we get the commutative diagram with exact rows

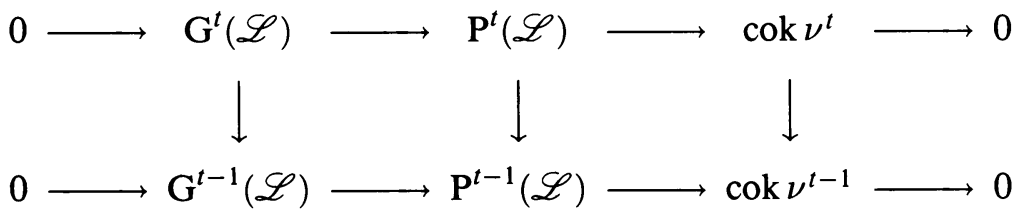

The vertical map on the right,

$$
\operatorname{cok} \nu^{t} \rightarrow \operatorname{cok} \nu^{t-1}
$$

is a surjection whose kernel we call the $t$-th inflection sheaf of $\phi$ and denote by inf $^{t}$. According to Theorem 2.3, it has length $\alpha_{t}$. The divisor corresponding to this kernel is the $t$-th inflection divisor defined in (B.1).

2.5. Degrees. In (B.4), we will give the standard definitions of the osculating developables, associated curves, and higher order dual varieties of a curve in projective space. The following result will give the degrees of these varieties.

Denote the kernel of $\mu^{t}$ by $\mathrm{E}^{t}(\mathscr{L})$, and consider the exact sequences

$$
0 \rightarrow \mathrm{E}^{t}(\mathscr{L}) \rightarrow V_{X} \stackrel{\mu^{t}}{\longrightarrow} \mathrm{G}^{t}(\mathscr{L}) \rightarrow 0
$$

and

$$
0 \rightarrow \mathrm{G}^{t}(\mathscr{L}) \rightarrow \mathrm{P}^{t}(\mathscr{L}) \rightarrow \operatorname{cok} \nu^{t} \rightarrow 0
$$

The degrees of $\mathrm{G}^{t}(\mathscr{L})$ and $\mathrm{E}^{t}(\mathscr{L})$ are an immediate consequence of Theorem 2.3. First we need some notation: if $F$ is any vector bundle on $X$, then define $c_{1}(F)$ to be the divisor class corresponding to the line bundle $\operatorname{det} F$; if $\mathscr{F}$ is a torsion sheaf on $X$, define [F] to be the class of the divisor $\sum_{x \in X}$ length $\left(\mathscr{F}_{X}\right) \cdot$ $x$.

Corollary 2.5.3. Let $X$ be a smooth projective curve of genus $g$, and assume the characteristic of $k$ is zero or greater than $t$ and the degree of $\mathscr{L}$. Then for $t \leq m=\operatorname{dim}_{k} \Gamma_{\phi}(V)-1$,

$$
c_{1}\left(\mathrm{G}^{t}(\mathscr{L})\right)=-c_{1}\left(\mathrm{E}^{t}(\mathscr{L})\right)=\left(\begin{array}{c}
t+1 \\
2
\end{array}\right) c_{1}\left(\Omega_{X / k}\right)+(t+1) c_{1}(\mathscr{L})-\left[\operatorname{cok} \nu^{t}\right]
$$

as divisor classes. In particular,

$$
\begin{aligned}
\operatorname{deg} \mathrm{G}^{t}(\mathscr{L}) & =-\operatorname{deg}\left(\mathrm{E}^{t}(\mathscr{L})\right)=\left(\begin{array}{c}
t+1 \\
2
\end{array}\right)(2 g-2)+(t+1) \operatorname{deg} \mathscr{L}-\sum_{i=1}^{t} \alpha_{i} \\
& =(t+1)(t g-t+\operatorname{deg} \mathscr{L})-\sum_{i=1}^{t} \alpha_{i} .
\end{aligned}
$$


Letting $t=m$, we get

$$
\sum_{i=1}^{m} \sum_{x \in X} \alpha_{i}(x) \cdot x=\left(\begin{array}{c}
m+1 \\
2
\end{array}\right) c_{1}\left(\Omega_{X / k}\right)+(m+1) c_{1}(\mathscr{L})
$$

as divisor classes, and taking degrees gives

$$
\begin{aligned}
\sum_{i=1}^{m} \alpha_{i} & =\left(\begin{array}{c}
m+1 \\
2
\end{array}\right)(2 g-2)+(m+1) \operatorname{deg} \mathscr{L} \\
& =(m+1)(m g-m+\operatorname{deg} \mathscr{L}) .
\end{aligned}
$$

Proof. The corollary follows directly from: Theorem 2.3 ; the Whitney sum formula applied to (2.5.1), (2.5.2), and (A.3.2); and the fact that if $\psi: E \rightarrow F$ is a generically surjective map between bundles of the same rank on a smooth projective curve, then

$$
c_{1}(E)-c_{1}(F)=[\operatorname{cok} \psi]
$$

(cf. [F, A.2.3]).

B.3. Examples. Here we present several standard examples. As a refinement of the theory, we consider the effect of covering maps and projections.

Example 3.1 [GH1, p. 270], [Pi1]). Suppose that $f$ has no inflectionary points. Under the assumptions of Corollary 2.5.3, this means that

$$
\sum_{i=1}^{m} \alpha_{i}=0=\left(\begin{array}{c}
m+1 \\
2
\end{array}\right)(2 g-2)+(m+1) \operatorname{deg} \mathscr{L} .
$$

This is only possible if $g=0$ and $\operatorname{deg} \mathscr{L}=m$, i.e., $\mathscr{L}=\mathscr{O}(m)$. Counting dimensions, this means that $\Gamma_{\phi}(V)=\Gamma\left(\mathbb{P}^{1}, \mathscr{O}(m)\right)$, and the map $f$ is just the embedding of $\mathbb{P}^{1}$ as a rational normal curve of degree $m$ in a linear subspace of dimension $m$ in $\mathbb{P}(V)$.

Remark 3.1.1. For a natural extension of the above example to a characterization of the Veronese embeddings of any $\mathbb{P}^{n}$, cf. [FKPT].

Example 3.2 (Weierstrass Points [GH1, p. 275]). Let $X$ have genus $g \geq 1$, and consider the canonical morphism

$$
f: X \rightarrow \mathbb{P}^{g-1}
$$

determined by the natural surjection

$$
\phi: \Gamma\left(X, \Omega_{X / k}\right)_{X} \rightarrow \Omega_{X / k} .
$$

Recall Remark 1.2. The inflectionary points for the canonical morphism are called Weierstrass points. By definition, $\ell_{x}(0)=g$, and since $\operatorname{deg} \Omega_{X / k}=$ $2 g-2$, we also have $\ell_{x}(2 g-1)=0$ for any point $x \in X$. Therefore, there are $g$ gap values at each point of $X$. From Corollary 2.5.3, the total weight of all the Weierstrass points, i.e., the sum of the weights at each point, is

$$
\begin{aligned}
\sum_{i=1}^{g}\left(a_{i}-i\right) & =\sum_{i=1}^{g} \alpha_{i-1} \\
& =\left(\begin{array}{l}
g \\
2
\end{array}\right)(2 g-2)+g(2 g-2) \\
& =(g-1) g(g+1) .
\end{aligned}
$$


Example 3.3 (Generalized Plücker Formulas [GH1, p. 270], [Pi1]). Assume that the characteristic of $k$ is zero or greater than $t+1$ and the degree of $\mathscr{L}$. Let $c_{1}\left(G^{i}(\mathscr{L})\right)$ denote the divisor class of the determinant of the osculating bundle of order $i$. From Corollary 2.5.3,

$$
c_{1}\left(\mathrm{G}^{t-1}(\mathscr{L})\right)-2 c_{1}\left(\mathrm{G}^{t}(\mathscr{L})\right)+c_{1}\left(\mathrm{G}^{t+1}(\mathscr{L})\right)=c_{1}\left(\Omega_{X / k}\right)-\sum_{x \in X} \alpha_{k+1}(x) \cdot x .
$$

Letting $d_{i}=\operatorname{deg} \mathrm{G}^{i}(\mathscr{L})$ and taking degrees in (3.3.1) yields

$$
d_{t-1}-2 d_{t}+d_{t+1}=2 g-2-\alpha_{k+1} \text {. }
$$

The expressions (3.3.2) for $t \geq 1$ are called the generalized Plücker formulas. The number $d_{i}$ is the degree of the osculating developable of order $i$, of the $i$-th associated map, and of the dual variety of order $i,(4.2,4.3)$.

Example 3.4 (Elliptic Curves). Let $\mathscr{L}$ be a line bundle of degree $n+1 \geq 3$ on an elliptic curve $E$. Consider the inflection numbers $\alpha_{i}, i=1 \ldots, n$, for the evaluation map

$$
\Gamma(E, \mathscr{L})_{E} \rightarrow \mathscr{L}
$$

By Riemann-Roch, $\alpha_{i}=0$ for $i<n$ and $\alpha_{n} \leq 1$ at each point, (cf. 1.2). (We can also see that $\alpha_{n} \leq 1$ by Remark 2.3.2.) Thus, Corollary 2.5.3 says that there are exactly $n+1$ inflectionary points. At each of the inflectionary points, $\alpha_{n}=1$.

Theorem 3.4.1 (Kato [Ka]). Choose any inflectionary point for (*) to be the identity in the group $E$. Then the inflectionary points are exactly the points of order $n+1$.

Proof. Let $p_{0}, \ldots, p_{n}$ be the inflectionary points, and take $p_{0}=0$ in the group $E$. By looking at the normal form, we see that the inflectionary points are exactly the points where a hyperplane of $\mathbb{P}(\Gamma(E, \mathscr{L}))$ meets the image of $E$ under the embedding determined by $(*)$ with multiplicity $n+1=\operatorname{deg} \mathscr{L}$, (1.3). Therefore, $\mathscr{L} \cong \mathscr{O}\left((n+1) p_{0}\right)$, and $(n+1) p_{i} \sim(n+1) p_{0}$ for each $i$. In other words, $(n+1) p_{i}=0$ for all $i$.

Remark 3.4.2. Assuming that the characteristic of $k$ is zero or greater than $n+1$, Corollary 2.5.3 and the comments made in the preceding example show that there are $(n+1)^{2}$ points of $E$ where $\alpha_{n}=1$. For an elliptic curve in the plane (i.e., $n=2$ ) in characteristic three, there are three or zero points of order three, depending on whether the curve is ordinary or supersingular, respectively, ([S, p. 106]). By Kato's theorem, this means that there are three or zero points where $\alpha_{2}=1$. In any case, the sum of the inflection numbers is not nine. This shows that the assumption on the characteristic in Theorem 2.3 and Corollary 2.5.3 is needed.

Example 3.5. Let $g_{d}^{r}$ be a generic nonspecial linear system of dimension $r$ and degree $d$ without base points on a smooth projective curve $X$ over the complex numbers. Let $f: X \rightarrow \mathbb{P}^{r}$ be the corresponding map with inflection numbers $\alpha_{i}$. Canuto, [Cn], shows that $\alpha_{i}=0$ for $i=1, \ldots, r-1$ for each point of $X$ and that there are exactly $(r+1)(r g-r+d)$ points where $\alpha_{r}=1$, otherwise $\alpha_{r}=0$. 
Example 3.6 (Coverings). Let $g: X \rightarrow Y$ be a finite, separable morphism of smooth projective curves over $k$. We want to relate the inflection numbers for a map of $Y$ into projective space with those of the induced mapping of $X$.

Suppose we are given a map

$$
f_{Y}: Y \rightarrow \mathbb{P}(V)
$$

with corresponding surjection to a line bundle

$$
\phi_{Y}: V_{Y} \rightarrow \mathscr{L}_{Y} .
$$

Composing $f_{Y}$ and $g$ gives

$$
f_{X}: X \rightarrow \mathbb{P}(V)
$$

and the corresponding surjection

$$
\phi_{X}: V_{X} \rightarrow \mathscr{L}_{X}
$$

where $\mathscr{L}_{X}=g^{*} \mathscr{L}_{Y}$.

Let $m+1=\operatorname{dim}_{k} \Gamma_{\phi_{Y}}(V)=\operatorname{dim}_{k} \Gamma_{\phi_{X}}(V)$, and for $i=1, \ldots, m$, let $\alpha_{X, i}$, $\alpha_{Y, i}$ be the inflection numbers for $\phi_{X}, \phi_{Y}$, respectively. The following result can be found in [Pe1]:

Proposition 3.6.1. Let $e_{x}$ be the ramification index at $x \in X$. Then

(1) For $x \in X$,

$$
\alpha_{X, i}(x)=e_{x} \cdot \alpha_{Y, i}(g(x))+e_{x}-1 .
$$

(2) Summing (1) over all points of $X$ gives the following relation for global inflection divisors:

$$
\sum_{x \in X} \alpha_{X, i} \cdot x=\operatorname{deg}(g) \sum_{x \in X} \alpha_{Y, i} \cdot x+i \cdot \sum_{x \in X}\left(e_{x}-1\right) \cdot x .
$$

Assume, in addition, that the characteristic of $k$ is either zero or greater than $t$ and the degree of $\mathscr{L}_{X}$. Then

(3) Letting $\mathrm{E}^{t}(\cdot)$ denote the kernel of the map to the osculating bundle $\mu^{t}$, the natural map $g^{*} \mathrm{P}^{t}\left(\mathscr{L}_{Y}\right) \rightarrow \mathrm{P}^{t}\left(\mathscr{L}_{X}\right)$ of (A.2.4) induces an isomorphism of exact sequences

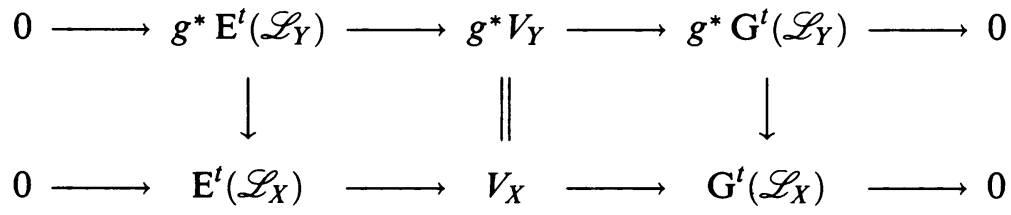

(4) For any bundle $F$ on $X$ or $Y$, let $c_{1}(\cdot)$ denote the divisor class of $\operatorname{det} F$. Then

$$
c_{1}\left(\mathrm{G}^{t}\left(\mathscr{L}_{X}\right)\right)=g^{*} c_{1}\left(\mathrm{G}^{t}\left(\mathscr{L}_{Y}\right)\right) .
$$

In particular,

$$
\operatorname{deg} G^{t}\left(\mathscr{L}_{X}\right)=\operatorname{deg}(g) \operatorname{deg} G^{t}\left(\mathscr{L}_{Y}\right) .
$$


(5) For $t \leq m$, the natural map $g^{*} \mathrm{P}^{t}\left(\mathscr{L}_{Y}\right) \rightarrow \mathrm{P}^{t}\left(\mathscr{L}_{X}\right)$ is generically surjective. The divisor corresponding to its cokernel is

$$
\left(\begin{array}{c}
t+1 \\
2
\end{array}\right) \cdot \sum_{x \in X}\left(e_{x}-1\right) \cdot x
$$

(6) For the inflection sheaves, (2.4), there is an exact sequence

$$
0 \rightarrow g^{*} \inf _{Y}^{t} \rightarrow \inf _{X}^{t} \rightarrow \Omega_{X / Y}^{\otimes t} \otimes \mathscr{L} \rightarrow 0 .
$$

Remark 3.6.2. Proposition 3.6.1 is closely related to the Riemann-Hurwitz Theorem. Summing (2) over $i$ yields

(*) $\quad \sum_{i=1}^{m} \sum_{x \in X} \alpha_{X, i} \cdot x=\operatorname{deg} g \sum_{i=1}^{m} \sum_{x \in X} \alpha_{Y, i} \cdot x+\left(\begin{array}{c}m+1 \\ 2\end{array}\right) \sum_{x \in X}\left(e_{x}-1\right) \cdot x$.

However, if we assume the characteristic of $k$ is either zero or greater than $m$ and the degree of $\mathscr{L}_{X}$, Corollary 2.5.3 says that

$$
\sum_{i=1}^{m} \alpha_{X, i}=\left(\begin{array}{c}
m+1 \\
2
\end{array}\right)(2 g(X)-2)+(m+1) \operatorname{deg} \mathscr{L}_{X}
$$

and similarly for $\sum \alpha_{Y, i}$. Substituting this into $(*)$ and simplifying gives the Riemann-Hurwitz Theorem:

$$
2 g(X)-2=\operatorname{deg} g(2 g(Y)-2)+\sum_{x \in X}\left(e_{x}-1\right) .
$$

Example 3.7 (Projections). Suppose that $V \subset \Gamma(X, \mathscr{L})$, and let $W$ be a subspace of $V$ of dimension $n=\operatorname{dim} V-1$ of globally generating sections. Consider the induced map

$$
\psi: W \subset V \stackrel{\phi}{\longrightarrow} \mathscr{L} .
$$

The corresponding map, $g: X \rightarrow \mathbb{P}(W)$, is obtained from the original map, $f$, by projection from a point. We can compare the inflectionary behavior of $f$ and $g$. (For the definition of an osculating space, used in the following proposition, cf. (B.4).)

Proposition 3.7.1. Let $\left\{\alpha_{i}\right\}_{i=1, \ldots, n}$ and $\left\{\beta_{i}\right\}_{i=1, \ldots, n-1}$ be the inflection numbers for $f$ and $g$, respectively. If the point of projection is contained in the osculating space of order $t$ at $x$ but not in an osculating space of order $t-1$, then

$$
\beta_{i}(x)= \begin{cases}\alpha_{i}(x), & \text { for } i=1, \ldots, t-1, \\ \alpha_{i+1}(x)+1, & \text { for } i=t, \ldots, n-1 .\end{cases}
$$

Proof. Choose a basis for $V, \sigma_{0}, \ldots, \sigma_{n}$, such that $\operatorname{ord}_{x}\left(\sigma_{i}\right)=i+\alpha_{i}$ and such that $\sigma_{0}, \ldots, \hat{\sigma}_{t}, \ldots, \sigma_{n}$ is a basis for $W$. Taking the corresponding normal forms for $f$ and $g,(1.3)$, we are projecting from the point $(0, \ldots, 1, \ldots, 0)$ -whose coordinates are all zero except for the $t$-th-onto the hyperplane $\left\{x_{t}=\right.$ $0\}$. Since the osculating space of order $i$ is given by $\left\{x_{i+1}=\cdots=x_{n}=0\right\}$, the result is clear.

Thus, the inflectionary behavior of the projected curve is the same as that for the original curve except at special points. If a point of the original curve has some osculating space that passes through the point of projection, the image will be "more" inflectionary. 
Example 3.8 (Osculating curves). Let $X$ be an irreducible plane curve, not necessarily smooth. Let $X(t, d)$ be the subset of the projective space of plane curves of degree $d$ consisting of curves meeting $X$ with multiplicity at least $t+1$. These curves are said to osculate $X$ with order $t$. To study $X(t, d)$, we linearize the problem, using the $d$-tuple Veronese embedding, $\nu_{d}: \mathbb{P}^{2} \rightarrow \mathbb{P}^{N}$, with $N=\frac{d(d+3)}{2}$. Let $\tilde{X}$ denote the normalization of $X$, and define the map

$$
\lambda_{d}: \tilde{X} \rightarrow X \subset \mathbb{P}^{2} \stackrel{\nu_{d}}{\longrightarrow} \mathbb{P}^{N} .
$$

The curves of degree $d$ osculating $X$ with order $t$ are in this way identified with hyperplanes of $\mathbb{P}^{N}$ that meet $\lambda_{d}(\tilde{X})$ with multiplicity at least $t+1$.

Basic results about $X(t, d)$ appear in [Pe1], including a refinement of Cayley's formula for the number of sextactic points on a plane curve, ([Ca]): those points where a conic meets the curve with multiplicity at least six.

B.4. Osculating spaces and higher order duals. We present the standard definitions of osculating spaces, developables, and associated maps, and we present a definition, due to Piene, [Pi2], of higher order dual varieties. We then give Piene's interpretation of these constructions using osculating bundles along with her calculation of the degrees of these constructions.

For the main results of the paper, it is only necessary to be familiar with the definitions, (4.1), and their interpretation via osculating bundles, Proposition 4.2.4.

As a new example, we consider a curve in projective space, and construct a map of the curve into a flag variety by considering the flag of osculating spaces of the curve at each point. We calculate the class of this curve in the intersection ring of the flag variety, (4.4).

Definition 4.1. (See Proposition 4.2.4 for the interpretation of the constructions presented here in terms of vector bundles.) With the notation as at the beginning of this appendix, assume that $f: X \rightarrow \mathbb{P}(V)$ spans a $\mathbb{P}^{m}$. For $t \leq m$, the osculating space of order $t$ at $x \in X$ is the unique $t$-plane having maximal order contact with $f(X)$ at $f(x)$ along the branch corresponding to $x$. Taking a normal form for $f$ at $x,(1.3)$, and letting $x_{0}, \ldots, x_{n}$ be the corresponding coordinates on $\mathbb{P}^{n}$, this $t$-plane is given by $\left\{x_{t+1}=\cdots=x_{n}=0\right\}$. For $t>$ $m$, define the osculating space of order $t$ to be the $\mathbb{P}^{m}$ spanned by $f$. The osculating developable of order $t$ of $f$ (or $f(X))$ is the union of the osculating spaces of order $t$.

Let $\operatorname{Osc}_{x}^{t}=\operatorname{Osc}_{x}^{t}(f)$ denote the osculating space of order $t$ at $x$. For $t \leq m$, associate each point of $X$ with its osculating $t$-plane in the Grassmannian of $t$-planes in $\mathbb{P}(V)$ to get the $t$-th associated map of $f$

$$
\begin{aligned}
f_{t}: X & \rightarrow G_{t} \mathbb{P}(V) \\
x & \mapsto \operatorname{Osc}_{x}^{t}(f) .
\end{aligned}
$$

The image of $f_{t}$ will be called the $t$-th associated curve of $f$.

The dual variety of order $t$ for $f$ is the set of hyperplanes-considered as a subset of the dual projective space $\mathbb{P}\left(V^{*}\right)$-containing some osculating space of order $t$. For $t>m$, the dual variety of order $t$ is just the $(n-m-1)$ dimensional linear space of hyperplanes containing the $\mathbb{P}^{m}$ spanned by $f$. 
Let $H_{x}^{t}=H_{x}^{t}(f)$ denote the set of hyperplanes containing the osculating space of order $t$ at $x$. Taking a normal form for $f$ at $x$ and coordinates on $\mathbb{P}^{n}$ as above, a hyperplane defined by $\sum_{i=0}^{n} a_{i} x_{i}$ is in $H_{x}^{t}$ if and only if $a_{0}=\cdots=a_{t}=0$. For $t \leq m$, define the $t$-th dual map of $f$ by

$$
\begin{aligned}
f^{t}: X & \longrightarrow G_{n-t-1} \mathbb{P}\left(V^{*}\right) \\
x & \mapsto H_{x}^{t} .
\end{aligned}
$$

The image of $f^{t}$ is called the $t$-th dual curve of $f$.

Remark 4.1.1. Let $t \leq m$. If $f$ is birational to its image, then the osculating space of order $t$ meets $f(X)$ at $f(x)$ along the branch corresponding to $x$ with multiplicity $t+1+\alpha_{t+1}$. For a general map, this number must be replaced by

$$
\frac{t+1+\alpha_{t+1}}{\operatorname{deg} f}
$$

Remark 4.1.2. If $X$ is not smooth, let $\pi: \tilde{X} \rightarrow X$ be the map from the normalization of $X$, and define all the constructions of Definition 4.1 for $f$ to be those of $f \circ \pi$. In terms of line bundles, we are replacing $\phi$ by

$$
\pi^{*} \phi: V_{\tilde{X}} \rightarrow \pi^{*} \mathscr{L} \text {. }
$$

Hence, if $X$ is embedded in $\mathbb{P}^{n}$ as a curve with singularities, this definition allows us to consider the osculating spaces of $X$ along its branches.

4.2. Modern viewpoint. In [Pi1], [Pi2], Piene has given the modern interpretation of the constructions of Definition 4.1 using her osculating bundles. This appears as Proposition 4.2.4, below.

Let $\mathrm{E}^{t}(\mathscr{L})$ denote the kernel of the natural map to the $t$-th order osculating bundle, $\mu^{t}$, and consider the exact sequence

$$
0 \rightarrow \mathrm{E}^{t}(\mathscr{L}) \rightarrow V_{X} \stackrel{\mu^{t}}{\longrightarrow} \mathrm{G}^{t}(\mathscr{L}) \rightarrow 0
$$

This induces maps of projective bundles

$$
\omega_{t}: \mathbb{P}\left(\mathrm{G}^{t}(\mathscr{L})\right) \rightarrow \mathbb{P}\left(V_{X}\right)=X \times \mathbb{P}(V) \stackrel{\pi_{2}}{\longrightarrow} \mathbb{P}(V)
$$

and

$$
\delta_{t}: \mathbb{P}\left(\mathrm{E}^{t}(\mathscr{L})^{*}\right) \longrightarrow \mathbb{P}\left(V_{X}^{*}\right)=X \times \mathbb{P}\left(V^{*}\right) \stackrel{\pi_{2}}{\longrightarrow} \mathbb{P}\left(V^{*}\right)
$$

where $\pi_{2}$ denotes the second projection in both cases.

Proposition 4.2.4. Assume that $X$ is a smooth projective curve and the characteristic of $k$ is zero or greater than $t$ and the degree of $\mathscr{L}$. Then the image of the fiber at $x, \omega_{t}\left(\mathbb{P}\left(\mathrm{G}^{t}(\mathscr{L})\right)_{x}\right)$, is the osculating space of order $t$ at $x$, and similarly, $\delta_{t}\left(\mathbb{P}\left(\mathrm{E}^{t}(\mathscr{L})^{*}\right)_{x}\right)=H_{x}^{t}$. Therefore, the image of $\omega_{t}$ is the osculating developable of order $t$, and the image of $\delta_{t}$ is the dual variety of order $t$.

For $t \leq m$, the map $X \rightarrow G_{t} \mathbb{P}(V)$ induced by $\mu^{t}: X \rightarrow \mathrm{G}^{t}(\mathscr{L})$ through the universal property of a Grassmannian is the $t$-th associated map. Similarly, the map $X \rightarrow G_{n-t-1} \mathbb{P}\left(V^{*}\right)$ induced by the natural surjection $V_{X}^{*} \rightarrow \mathrm{E}^{t}(\mathscr{L})^{*}$ is the $t$-th dual map of $f$. 
Corollary 4.2.5. With the assumptions of Proposition 4.2.4, the osculating developable and dual variety of order $t$ are irreducible.

Proof. The osculating developable of order $t$ is the image of $\mathbb{P}\left(\mathrm{G}^{t}(\mathscr{L})\right)$, and the dual variety of order $t$ is the image of $\mathbb{P}\left(\mathrm{E}^{t}(\mathscr{L})^{*}\right)$.

Remark 4.2.6. Let $X \subset \mathbb{P}^{n}$ be a smooth embedding of a curve in projective space determined by sections of a line bundle $\mathscr{L}$. (Note: The discussion given here is easily generalized to the case $\operatorname{dim} X>1$.) Define

$$
Y=\left\{(x, H) \in X \times\left(\mathbb{P}^{n}\right)^{*} \mid T_{x} X \subset H\right\}
$$

where $T_{x} X$ is the embedded tangent space to $X$ at $x$. Letting $N_{X} \mathbb{P}^{n}$ be the normal bundle to $X$ in $\mathbb{P}^{n}$, we have that $Y$ is isomorphic to $\mathbb{P}\left(N_{X} \mathbb{P}^{n}\right)$ over $X,([\mathrm{~F}, 3.2 .21])$. The dual variety to $X$ is usually defined to be the image of the projection $Y \rightarrow\left(\mathbb{P}^{n}\right)^{*}$. However, since $T_{X} X$ is just the first osculating space at $x$, the dual variety coincides with our dual variety of order one. In fact, Kleiman, [K1], shows that in our situation, $\mathrm{E}^{1}(\mathscr{L}) \cong\left(N_{X} \mathbb{P}^{n}\right)^{*} \otimes \mathscr{L}$. Since $\mathscr{L}$ is a line bundle,

$$
\mathbb{P}\left(\mathrm{E}^{1}(\mathscr{L})^{*}\right)=\mathbb{P}\left(N_{X} \mathbb{P}^{n} \otimes \mathscr{L}^{*}\right) \cong \mathbb{P}\left(N_{X} \mathbb{P}^{n}\right) .
$$

4.3. Degree of the osculating developable and the higher order dual. The next proposition is Piene's computation of the degrees of the osculating developables and higher order dual varieties as cycles in the intersection ring of projective space.

Proposition 4.3.1. Let $t<m$. Let $X$ be a smooth projective curve of genus $g$, and assume that the characteristic of $k$ is zero or greater than $t$ and the degree of $\mathscr{L}$. Then the dimension of the osculating developable of order $t$ is $t+1$, and the dimension of the higher order dual variety of order $t$ is $n-t$. In other words,

$$
\operatorname{dim}\left(\operatorname{im} \omega_{t}\right)=t+1, \quad \operatorname{dim}\left(\operatorname{im} \delta_{t}\right)=n-t .
$$

Further,

$$
\begin{aligned}
\operatorname{deg}\left(\omega_{t}\right) \operatorname{deg}\left(\operatorname{im} \omega_{t}\right) & =\operatorname{deg}\left(\delta_{t}\right) \operatorname{deg}\left(\operatorname{im} \delta_{t}\right) \\
& =\operatorname{deg}\left(f_{t}\right) \operatorname{deg}\left(\operatorname{im} f_{t}\right) \\
& =\operatorname{deg}\left(f^{t}\right) \operatorname{deg}\left(\operatorname{im} f^{t}\right) \\
& =d_{t}=\operatorname{deg} G^{t}(\mathscr{L}) \\
& =\left(\begin{array}{c}
t+1 \\
2
\end{array}\right)(2 g-2)+(t+1) \operatorname{deg} \mathscr{L}-\sum_{i=0}^{t} \alpha_{i}
\end{aligned}
$$

where $\operatorname{deg} \omega_{t}$ is the degree of the map from the domain to the image of $\omega_{t}$ and similarly for $\delta_{t}, f_{t}$, and $f^{t}$.

Remark 4.3.2. Proposition 3.3.1 of the main body of the paper shows that if the characteristic of $k$ is equal to zero or is larger than $t$ and $d_{t}=\operatorname{deg} G^{t}(\mathscr{L})$, and if $f$ is birational to its image, then $f_{t}$ is birational to its image, i.e., the generic osculating space of order $t$ is the osculating space of order $t$ at only one point of $f(X)$. Similarly, $f^{t}$ is birational to its image.

The birationality of $\omega_{t}$ and $\delta_{t}$ seems to be a more difficult question. One would not expect $\omega_{m-1}$ to be birational. For example, the tangent developable 
to a plane curve fills the whole plane; the generic point on a tangent line will lie on other tangent lines as well. (On the other hand, since $f_{m-1}$ is birational to its image, so is $\delta_{m-1}$.) What if $t<m-1$ ? This would imply the trisecant lemma: that the generic secant of a nonplanar curve does not meet the curve again. For a proof of the trisecant lemma, cf. [L2, Lemma 15].

Example 4.4 (Flags). At each point $x \in X$, the osculating spaces form a flag of linear subspaces of projective space,

$$
\{x\} \subset \operatorname{Osc}_{x}^{1} \subset \operatorname{Osc}_{x}^{2} \subset \cdots
$$

Associating a point with its corresponding flag, in this way, determines a map of the curve into the variety of flags in projective space. We will compute the class of this curve in the intersection ring of the variety of flags. For simplicity, assume that $f: X \rightarrow \mathbb{P}^{n}$ is birational to its image and spans $\mathbb{P}^{n}$.

Let $F$ be the variety of complete flags in $\mathbb{P}^{n},([F, 14.7 .16])$. The points of $F$ are the flags of linear subspaces of $\mathbb{P}^{n}$

$$
L_{0} \subset L_{1} \subset \cdots \subset L_{n-1}
$$

where $\operatorname{dim} L_{i}=i$. Fix such a flag $\pi_{0} \subset \pi_{1} \subset \cdots \subset \pi_{n-1}$. A basis for the intersection ring of $F$ in dimension one is

$$
\ell_{j}=\left\{\left(L_{0}, \ldots, L_{n-1}\right) \mid L_{i}=\pi_{i} \text { for } i \neq j, L_{j} \subset \pi_{j+1}\right\}
$$

for $j=0, \ldots, n-1$. The dual basis in codimension one is

$$
\ell_{j}^{*}=\left\{\left(L_{0}, \ldots, L_{n-1}\right) \mid L_{i} \cap \pi_{n-i-1} \neq \varnothing\right\} .
$$

Define

$$
\begin{aligned}
\tilde{f}: X & \rightarrow F \\
x & \mapsto\left(x, \operatorname{Osc}_{x}^{1}, \ldots, \operatorname{Osc}_{x}^{n-1}\right) .
\end{aligned}
$$

The class of $\tilde{f}_{*}(X)$ in the intersection ring for $F$ is

$$
\left[\tilde{f}_{*}(X)\right]=\sum_{i=0}^{n-1}\left(\tilde{f}_{*}(X) \cdot \ell_{i}^{*}\right) \ell_{i} .
$$

Assume that the associated maps, $f_{t}$, are birational to their images; for example, we could assume that the characteristic of $k$ is zero or large enough (Proposition 3.3.1 of main body of the paper). Then, $\tilde{f}_{*}(X) \cdot \ell_{i}^{*}$ is the number of osculating spaces of order $i$ meeting a generic $(n-i-1)$-plane; in other words, it is the degree of the osculating developable and the associated map, $d_{t},(4.3 .1)$. Thus, we find,

$$
\left[\tilde{f}_{*}(X)\right]=\sum_{i=0}^{n-1} d_{t} \ell_{i}
$$

B.5. Piene duality theorem. The purpose of this section is to state Piene's duality theorem for curves in projective space. This result, found in [Pi1], is the modern expression of the duality theorems of the nineteenth century for curves in projective space. A main result of our paper is an extension of her duality theorem to one for curves in Grassmannians. 
Let $V \subset \Gamma(X, \mathscr{L})$ be a vector space of dimension $n+1$ of generating sections of a line bundle $\mathscr{L}$ on $X$. The corresponding map, $f: X \rightarrow \mathbb{P}(V)$, spans $\mathbb{P}(V)$. In (B.4), we defined the $t$-th associated map

$$
f_{t}: X \rightarrow G_{t} \mathbb{P}(V)
$$

sending a point to its osculating space of order $t$, and we defined the $t$-th dual map

$$
f^{t}: X \rightarrow G_{n-t-1} \mathbb{P}\left(V^{*}\right)
$$

sending a point $x \in X$ to the linear space of hyperplanes containing the osculating space of order $t$ at $x$.

Definition 5.1. The dual of $f$ is the map

$$
\begin{aligned}
f^{*}: X & \longrightarrow \mathbb{P}\left(V^{*}\right) \\
x & \mapsto \operatorname{Osc}_{x}^{n-1}
\end{aligned}
$$

sending a point $x \in X$ to the osculating hyperplane at $x$. This dual is the $(n-1)$-th dual map of (B.4), i.e., $f^{*}=f^{n-1}$.

We saw in Proposition 4.2.4, with an assumption on the characteristic of $k$, that the associated map corresponds to the map of vector bundles

$$
\mu^{t}: V_{X} \rightarrow \mathrm{G}^{t}(\mathscr{L})
$$

There is an exact sequence

$$
0 \rightarrow \mathrm{E}^{t}(\mathscr{L}) \rightarrow V_{X} \stackrel{\mu^{t}}{\longrightarrow} \mathrm{G}^{t}(\mathscr{L}) \rightarrow 0
$$

and the $t$-th dual map, $f^{t}$, corresponds to the natural surjection

$$
V_{X}^{*} \rightarrow \mathrm{E}^{t}(\mathscr{L})^{*} \text {. }
$$

By Theorem $2.3, \mathrm{G}^{t}(\mathscr{L})$ has rank $t+1$ for $t \leq n$. Therefore, $\mathrm{E}^{n-1}(\mathscr{L})$ is a line bundle.

Theorem 5.2 (Piene Duality Theorem, [Pi1]). Let $X$ be a smooth projective curve, and assume that the characteristic of $k$ is zero or greater than $n$ and the degree of $\mathscr{L}$. Then the $t$-dual map of the dual map, $f^{*}$, is the $(n-t-1)$-th associated map of $f$. In symbols,

$$
\left(f^{*}\right)^{t}=f_{n-t-1}
$$

In particular, the double dual of $f$ is $f$, itself:

$$
\left(f^{*}\right)^{*}=f \text {. }
$$

For more discussion, see $\S 7$ of the main body of the paper.

\section{REFERENCES}

[AK] A. Altman and S. Kleiman, Introduction to Grothendieck Duality Theory, Lecture Notes in Math., vol. 146, Springer, Berlin, Heidelberg, New York, 1970.

[Ba1] E. Ballico, Weierstrass loci for vector bundles on curves, preprint.

[Ba2] _ On the differential properties of algebraic morphisms into Grassmannians, preprint.

[Br] A. Bruguières, Maps of elliptic curves to Grassmannians, Compositio Math. 63 (1987), 15-40. 
[Ca] A. Cayley, On the sextactic points of a plane curve, Philos. Trans. 155 (1865), 545-578.

[Cn] G. Canuto, Associated curves and Plücker formulas in Grassmannians, Invent. Math. 53 (1979), 77-90.

[E] W. L. Edge, The theory of ruled surfaces, Cambridge Univ. Press, London, 1931.

[F] W. Fulton, Intersection theory, Springer-Verlag, Berlin, Heidelberg, New York, 1984.

[FKPT] W. Fulton, S. Kleiman, R. Piene, and H. Tai, Some intrinsic and extrinsic characterizations of the projective space, Bull. Soc. Math. France 113 (1985), 205-210.

[G] A. Grothendieck and J. A. Dieudonné, Éléments de géométrie algébrique, IV, Étude locale des schémas et des morphismes de schémas, Inst. Hautes Etudes Sci. Publ. Math., No. 32 (1967), 39-46.

[GH1] P. Griffiths and J. Harris, Principles of algebraic geometry, Wiley-Interscience, New York, 1978.

[GH2] Algebraic geometry and local differential geometry, Ann. Sci. École. Norm. Sup. (4) 12 (1979), 355-432.

[H] R. Hartshorne, Algebraic geometry, Springer-Verlag, Berlin, Heidelberg, New York, 1977.

[J] N. Jacobson, Basic algebra I, Freeman, San Francisco, Calif., 1980.

[Ka] A. Kato, Singularities of the projective embedding (points of order $n$ on an elliptic curve), Nagoya Math. J. 45 (1971), 97-107.

[K1] S. Kleiman, The enumerative theory of singularities, Real and Complex Singularities, Oslo 1976, (P. Holm, ed.), Sijthoff and Noordhoff, 1977, pp. 297-396.

[K2] Concerning the dual variety, 18th Scandanavian Congress of Math., Aarhus 1980, (E. Balslev, ed.), Progress in Math 11, Birkhäuser, Boston, 1980, pp. 386-396.

[L1] D. Laksov, Wronskians and Plücker formulas for linear systems on curves, Institut Mittag-Leffler, Report No. 11 (1981).

[L2] - Some enumerative properties of secants to nonsingular projective schemes, Math. Scand. 39 (1976), 171-190.

[M] H. Matsumura, Commutative ring theory, Cambridge Univ. Press, London, 1986.

[Pe1] D. Perkinson, Jet bundles and curves in Grassmannians thesis, University of Chicago, 1990.

[Pe2] _ Principal parts of line bundles on toric varieties, Matematisk Institutt, University of Oslo, preprint.

[Pi1] R. Piene, Numerical characters of a curve in projective n-space, Real and Complex Singularities, Oslo 1976, (P. Holm, ed.), Sijthoff and Noordhoff, 1977, pp. 475-495.

[Pi2] _ A note on higher order dual varieties with an application to scrolls, Proc. Sympos. Pure Math., vol. 40, Amer. Math. Soc., Providence, RI, 1983, pp. 335-342.

[PS] Duality for rational normal scrolls, Comm. Algebra 12 (1984), 1041-1066.

[S] J. Silverman, The arithmetic of elliptic curves, Springer-Verlag, Berlin, Heidelberg, New York, 1986.

Department of Mathematics, Reed College, Portland, Oregon 97202

E-mail address: davidp@reed.edu 